\title{
КИБЕРНЕ ТИКА
}

\section{И ВЫЧИСЛИТЕ ЛЬНАЯ}

\section{TEXHИKA}

CYBERNETICS AND COMPUTER ENGINEERING

\section{Выпуск 180}

Основан в 1965 г.

Периодичность: 4 раза в год

\section{СОДЕРЖАНИЕ}

\section{Информатика и информационные технологии}

Суровцев И.В. Преобразование структуры данных при определении концентрации методами инверсионной хронопотенциометрии .................. 4

Доценко С.И. Решение задачи оптимального выбора с групповым просмотром с помощью теоретико-игрового подхода …........................... 15

Савченко E.A. Технология решения задачи моделирования и прогнозирования на основе индуктивного подхода

\section{Интеллектуальное управление и системы}

Мащенко С.О., Шушарин Ю.В. Критерий минимаксного сожаления в задаче принятия решений с нечетким множеством состояний окружающей среды

Павлов В.В., Волков А.Е., Волошенюк Д.А. Инвариантная сетецентрическая система управления конфоликтными ситуациями воздушных кораблей на этапе захода на посадку

Коцур М.П., Наконечный А.Г. Оптимальное управление нестационарным режимом каскадного термоэлектрического охладителя

Медицинская и биологическая кибернетика

Чернышенко С.В., Рузич Р.В. Дискретные эффректы в непрерывных моделях сукцессионных процесов 


\title{
КИБЕРНЕ ТИКА
}

\section{И ВЫЧИСЛИТЕ ЛЬНАЯ}

\section{TEXHИКA}

\author{
CYBERNETICS AND COMPUTER ENGINEERING
}

Issue 180

\section{TABLE OF CONTENTS}

Informatics and Information Technologies

Surovtsev I.V. Transformation of Data Structure in Determining the Concentration by Methods of Inversion Chronopotentiometry

Dotsenko S.I. Solution of the Problem of Optimal Choices with a Group Browsing by a Game-theoretic Approach

Savchenko E.A. The Technology for Solving the Problem of Modeling and Forecasting Based on Inductive Approach

Intellectual Control and Systems

Mashchenko S.O., Shusharin Yu.V. Minimax Regret Criterion in Decision Making Problems with the Fuzzy Set of States of the Environment.

Pavlov V.V., Volkov A.E., Voloshenyuk D.A. Invariant Net-Centric Control System for Conflict Avoidance of Aircrafts in the Landing Phase

Kotsur M.P., Nakonechniy A.G. Optimal Control of Non-stationary Regime of Cascade Thermoelectric Cooler

Medical and Biological Cybernetics

Chernyshenko S.V., Ruzich R.V. Discrete Effects in Continuous Models of Successions

Our authors 


\section{КIБЕРНЕ ТИКА}

\section{ТА ОБЧИСЛЮВАЛЬНА}

\section{TEXHIKA}

CYBERNETICS AND COMPUTER ENGINEERING

\section{Випуск 180}

\section{MICT}

Інформатика та інформаційні технології

Суровцев І.В. Перетворення структури даних при визначенні концентрації методами інверсійної хронопотенціометрії

Доценко С.I. Розв'язок задачі оптимального вибору з груповим переглядом за допомогою теоретико-ігрового підходу

Савченко С.А. Технологія розв'язання задачи моделювання та прогнозування на основі індуктивного підходу

Інтелектуальне керування та системи

Мащенко С.О., Шушарін Ю.В. Критерій мінімаксного жалю в задачі прийняття рішень з нечіткою множиною станів довкілля

Павлов В.В., Волков А.С., Волошенюк Д.О. Інваріантна мережецентрична система керування конфлліктними ситуаціями повітряних кораблів на етапі заходу на посадку

Коцур М.П., Наконечний О.Г. Оптимальне керування нестаціонарним режимом каскадного термоелектричного охолоджувача

Медична та біологічна кібернетика

Чернишенко С.В., Рузич Р.В. Дискретні ефекти в неперервних моделях сукцесійних процесів

Інформація про авторів 95 


\title{
Информатика и
}

\section{информационные технологии}

УДК 004.67: 004.023

\section{ПРЕОБРАЗОВАНИЕ СТРУКТУРЫ ДАННЫХ ПРИ ОПРЕДЕЛЕНИИ КОНЦЕНТРАЦИИ МЕТОДАМИ ИНВЕРСИОННОЙ ХРОНОПОТЕНЦИОМЕТРИИ}

\author{
И.В. Суровцев
}

Международный научно-учебный центр информационных технологий и систем НАН Украины и МОН Украины

Рассматривается новая информационная технология измерения концентрации токсичных элементов, которая позволяет повысить чувствительность и достоверность определения концентрации элементов в растворе методами инверсионной хронопотенциометрии. Перед началом анализа выполняется целенаправленное преобразование структуры данных измерения, которое состоит из гистограммной цифровой фильтрации и дифференцирования обратного сигнала. Полученный сигнал рассматривают как линейную сумму моделей, которые описываются функциями нормального распределения. Созданная информационная технология имеет универсальный характер и может быть применена для анализа сигналов различной природы, в которых значения монотонно возрастают или убывают.

Ключевые слова: преобразование структуры данных, методы инверсионной хронопотенциометрии, моделирование, информационная технология.

Розглядається нова інформаційна технологія вимірювання концентрації токсичних елементів, яка дозволяє збільшити чутливість та достовірність визначення концентрації елементів у розчині методами інверсійної хронопотенціометрії. Перед початком аналізу виконується цілеспрямоване перетворення структури вихідних даних вимірювання, яке складається 3 гістограмної цифрової фільтрації та диференціювання зворотного сигналу. Одержаний сигнал розглядають як лінійну суму моделей, які описуються функціями нормального розподілу. Створена інформаційна технологія має універсальний характер та може бути застосована для аналізу сигналів різної природи, в яких значення монотонно збільшуються або зменшуються.

Ключові слова: перетворення структури даних, методи інверсійної хронопотенціометрії, моделювання, інформаційна технологія.

\section{ВВЕДЕНИЕ}

Оценку состояния окружающей среды производят путем постоянного экологического мониторинга отдельных ее объектов. Одним из факторов экологической безопасности является определение в них концентрации ряда токсичных элементов (ртуть, мышьяк, свинец, кадмий и др.). Их величина для каждого объекта нормируется законодательными актами в виде предельно допустимых концентраций (ПДК). 
Концентрацию элементов определяют различными физико-химическими методами анализа. Наиболее чувствительными являются электрохимические способы исследований - вольтамперометрические и хронопотенциометрические методы, которые позволяют определять микроконцентрации (следы) токсичных элементов. По сравнению с вольтамперометрическим анализом использование методов инверсионной хронопотенциометрии (ИХП) позволяет значительно проще реализовать их технически, увеличить чувствительность, повторяемость и надежность измерений. Теоретическое обоснование использования метода ИХП в Украине было заложено академиком Скобцом Е.М. [1], большой вклад в развитие метода внесли Карнаухов А.И., Копилевич В.А., Галимова В.М. и др. [2]. Методы ИХП получили широкое распространение в практике аналитических исследований у нас в стране и за рубежом.

Суть измерения концентрации методом ИХП состоит в следующем. На объекте окружающей среды отбирают пробу и подготавливают ее к анализу в виде раствора пробы. Для измерения используют электрохимическую ячейку (стаканчик, установленный на магнитную мешалку, в который опускают измерительный и вспомогательный электрод). Для каждого элемента сначала в фоновом растворе (Фон), затем в растворе пробы (Проба) определяют время инверсии или выхода ионов из измерительного электрода после их предварительного концентрирования при перемешивании. В раствор пробы добавляют известную массу стандартного иона исследуемого элемента и снова определяют время инверсии в растворе (Добавка). По найденным величинам времени инверсии, которое прямо пропорционально концентрации ионов элемента в растворе пробы, и параметрам анализируемой пробы определяют значения концентрации токсичного элемента в объекте окружающей среды.

\section{ПОСТАНОВКА ЗАДАЧИ}

Основной количественной величиной, определяемой по исходному сигналу методом ИХП, является время инверсии анализируемого элемента, а качественной - диапазон изменения потенциалов инверсии в выбранном растворе фонового электролита. Для проведения анализа подбираются такие электрохимические переменные измерения, чтобы исключить или уменьшить влияние на сигнал других элементов и более точно определить время инверсии. При выполнении анализа реальных объектов исходный сигнал представляет собой интегральную сумму многокомпонентного измерения, где каждый компонент представляет собой процесс инверсии отдельного элемента.

Непосредственное измерение времени инверсии по исходному сигналу по точкам перегиба возможно только для больших концентраций элементов - больших, чем 1 мкг/мл в растворе пробы [1]. Иной способ определения времени инверсии был предложен Цепковым Г.В. и др. [3]. Согласно этому способу исходный сигнал последовательно превращался в разнополярные прямоугольные и единичные по амплитуде импульсы, продолжительность которых пропорциональна концентрации ионов в

(C) И.В. Суровцев, 2015 
растворе. Проверка на практике показала, что таким способом можно определять концентрацию не ниже 0,1 мкг/мл. Технические и математические сложности непосредственного измерения времени по сигналу долгое время тормозило использование метода ИХП на практике, поскольку необходимо было выполнять измерение концентраций на уровне ниже ПДК, порядка 0,001 мкг/мл. Таким образом, определение времени инверсии отдельных компонентов непосредственно из исходного сигнала или невозможно, или приводит к большим погрешностям измерений. С появлением персональных компьютеров решение этой проблемы стало возможным благодаря предварительному считыванию всего исходного сигнала инверсии в память и дальнейшей его обработке.

Одним из эффективных приемов решения технических задач анализа сложных многомерных данных или многокомпонентных сигналов является использование индуктивных методов теории редукции, позволяющих преобразовать структуру данных и значительно упростить их. Индуктивные методы лежат в основе решения практически всех задач экстраполяции, таких как задачи обучения распознавания образов, восстановления функций, косвенных измерений, прогноза и обнаружения закономерностей, а теория редукции позволяет указать на условия, при которых гарантируются качество и надежность решения этих задач.

Наиболее весомый вклад в создание и развитие теории редукции принадлежит профессору Васильеву В.И. [4]. Дальнейшие исследования учеников созданной им научной школы были направлены на развитие и практическое использование теории редукции при решении технических и экономических задач [5].

Согласно основному принципу этой теории необходимо предварительно сформировать такое пространство координат, в котором разделение на образы или компоненты происходит наиболее явно. Нужно стремиться к предельному упрощению информации, полученной в процессе наблюдения, ее сокращению и линеаризации, оставляя только то, что способствует решению задачи. Так, при решении многомерных задач такой подход позволяет снизить размерность пространства и выбрать наиболее существенные признаки, позволяющие решить поставленную задачу.

Анализ проблемы определения концентрации элементов методами инверсионной хронопотенциометрии показал, что исходный сигнал, который представляет собой интегральную сумму потенциалов инверсии компонентов измерения во времени, необходимо предварительно преобразовать в более простой вид. Для этого нужно осуществить целенаправленную редукцию структуры данных, т.е. такое преобразование системы координат, которое позволит представить исходный сигнал в виде линейной суммы отдельных компонентов измерения. Построив математическую модель отдельного компонента, можно по ней более качественно определить значение времени инверсии элемента, а следовательно, гораздо точнее подсчитать значение концентрации анализируемого элемента в пробе объекта.

Такая последовательность преобразования структуры исходных данных и построения математических моделей инверсии отдельных компонентов измерения дала бы возможность существенно увеличить чувствительность, 
точность и повторяемость определения концентрации токсичных элементов методами инверсионной хронопотенциометрии.

Цель работы - создать информационную технологию измерения концентрации токсичных элементов методами ИХП, которая позволит существенно увеличить чувствительность и надежность определения следовых значений массовой концентрации токсичных элементов в жидких пробах разных объектов окружающей среды.

\section{ИНФОРМАЦИОННАЯ ТЕХНОЛОГИЯ ИЗМЕРЕНИЯ КОНЦЕНТРАЦИИ ТОКСИЧНЫХ ЭЛЕМЕНТОВ В ЖИДКИХ ПРОБАХ}

Общая последовательность обработки исходных данных в информационной технологии (ИТ) измерения концентрации методами ИХП может быть представлена в следующем виде:

Эman I. Преобразование структуры исходных данных.

Эman II. Моделирование отдельных компонентов и определение времени инверсии.

Эman III. Расчет значения концентрации анализируемого элемента.

Для рассмотрения особенностей выполнения последовательности обработки данных в ИТ измерения концентрации используем в качестве примера измерение значения концентрации меди в реальном растворе пробы способом добавки стандартного образца ионов меди методом ИХП. Для этого выполним измерение сигналов Фона, Пробы и Добавки. Результаты трех измерений представлены на рис. 1.

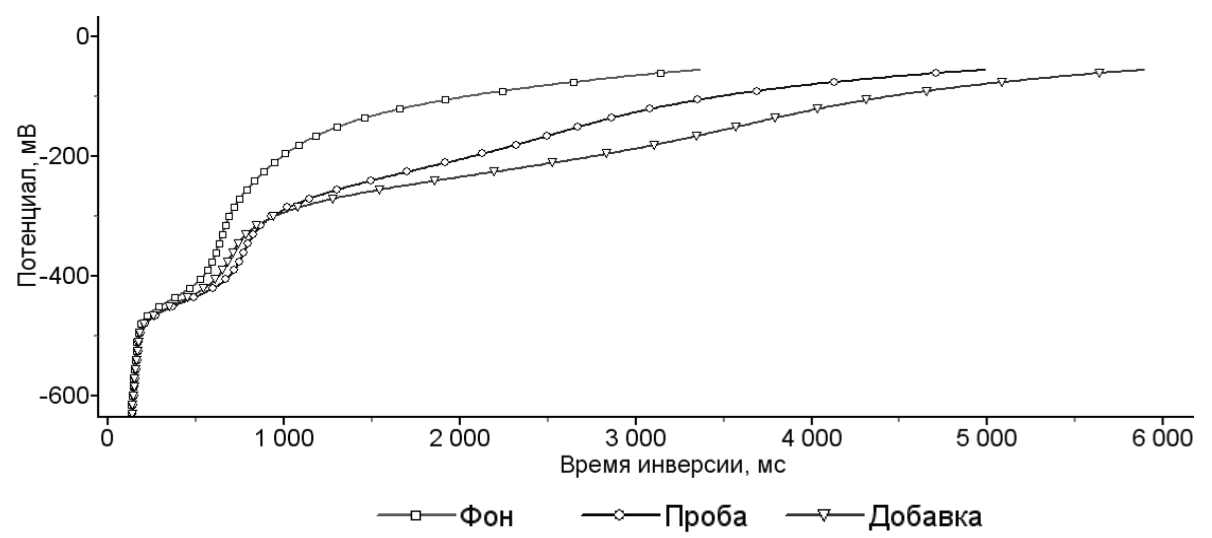

Рис. 1. Измерение потенциалов инверсии меди в присутствии ионов свинца

Прокомментируем результаты измерений:

- $\quad$ в сигнале Фона обнаружены ионы свинца (в диапазоне от -500 мВ до -350 мВ), которые практически повторяются в сигналах Пробы и Добавки;

- $\quad$ в сигнале Фона ионы меди (в диапазоне от -350 мВ до -100 мВ) практически отсутствуют, но имеются в сигнале Пробы и выросли после добавки стандартного образца ионов меди в Добавке.

Анализ внешнего вида исходных сигналов на рис. 1 подтверждает значительную сложность непосредственного измерения времени инверсии отдельных компонентов. 


\section{ЭТАП І. ПРЕОБРАЗОВАНИЕ СТРУКТУРЫ ИСХОДНЫХ ДАННЫХ}

Пусть исходный сигнал $f(t)$, поступающий от измерительного и вспомогательного электрода, представляет собой интегральную сумму последовательного измерения потенциалов инверсии отдельных компонентов во времени.

Выполним преобразование системы координат исходных данных, которое позволит представить исходный сигнал в виде линейной суммы отдельных компонентов измерения инверсии, в такой последовательности:

- $\quad$ исходный сигнал $f(t)$ очищают от шумов измерения методом цифровой фильтрации [6] и сглаживают для получения монотонного возрастающего сигнала;

- $\quad$ сигнал $f(t)$ преобразуют в обратный сигнал $t(f)$ с шагом дискретизации $\Delta f$;

- $\quad$ обратный сигнал $t(f)$ дифференцируют и получают сигнал интенсивности $d t / d f$ по потенциалу $f$.

Таким образом, преобразование структуры данных состоит в изменении системы координат

$$
(f, t) \rightarrow(d t / d f, f) .
$$

Полученный сигнал интенсивности времени изменения потенциала $d t / d f$ по потенциалу $f$ для нашего примера представлен на рис. 2.

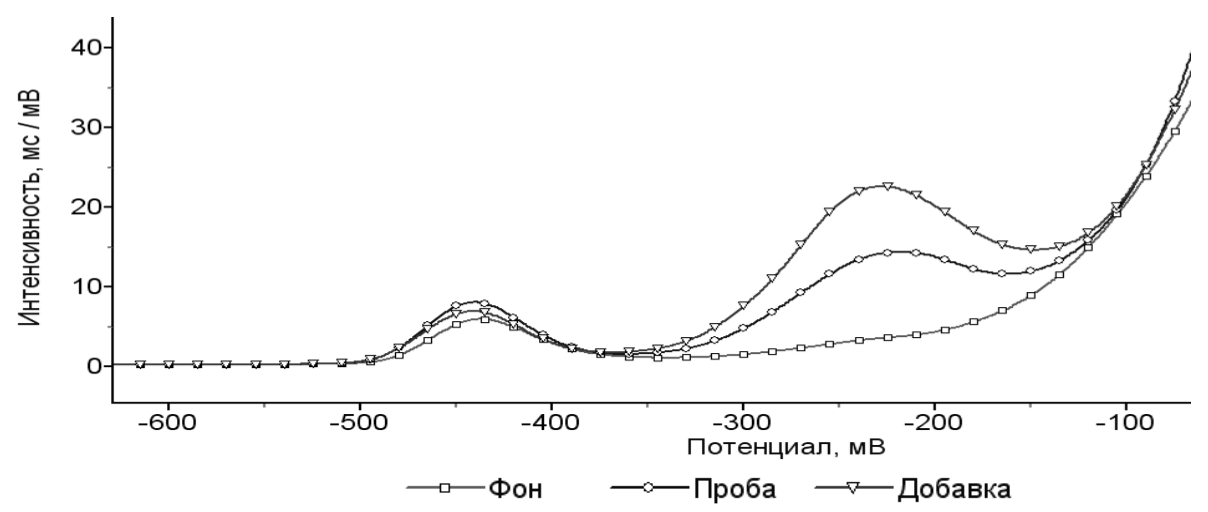

Рис. 2. Интенсивность изменения потенциалов

\section{ЭТАП ІІ. МОДЕЛИРОВАНИЕ ОТДЕЛЬНЫХ КОМПОНЕНТОВ И ОПРЕДЕЛЕНИЕ ВРЕМЕНИ ИНВЕРСИИ}

После выполнения преобразования структуры исходных данных сигнал интенсивности представляет собой линейную сумму компонентов измерения инверсии.

Время инверсии измерения каждого элемента определяется как площадь отдельного компонента сигнала интенсивности $\Delta T(f)$ в диапазоне его измерения: 


$$
t_{j}=\int_{f_{1 j}}^{f_{2 j}} \Delta T(f) d f=\sum_{i=f_{1 j}}^{f_{2 j}} \Delta T\left(f_{i}\right),
$$

где $t_{j}$ - время инверсии; $f_{1 j}, f_{2 j}-$ значения потенциалов диапазона измерения $j$-го компонента.

Для более точного определения времени инверсии измерения элемента каждый отдельный компонент сигнала интенсивности описывают в виде математической модели. Основная цель моделирования - наиболее точное определение значения времени инверсии (площади сигнала интенсивности), по которому будет вестись расчет концентрации элемента. Компонент сигнала интенсивности аппроксимируют подходящей функцией, исходя из индуктивных предположений и опыта разработчика.

Как показали исследования, компоненты интенсивности хорошо описываются функциями нормального распределения с разными дисперсиями [7]. В случае тесной взаимосвязи моделей компонентов в сигнале интенсивности, как в нашем примере, используют итерационную процедуру синтеза моделей для разделения суммы пересекающихся компонентов.

Точность построения каждой модели контролируют по значению среднеквадратической ошибки аппроксимации. Оценку точности моделирования всего сигнала интенсивности осуществляют следующим образом:

- $\quad$ определяют модельную интегральную сумму сигнала $t_{m}(f)$ (последовательным суммированием компонент);

- $\quad$ сигнал $t_{m}(f)$ преобразуют в обратный модельный сигнал $f_{m}(t)$;

- $\quad$ считают, что процесс моделирования удовлетворительный, если выполняется условие

$$
\max \left|f_{m}(t)-f(t)\right|<\varepsilon,
$$

где $\varepsilon-$ заданный критерий качества.

\section{ЭТАП III. РАСЧЕТ ЗНАЧЕНИЯ КОНЦЕНТРАЦИИ АНАЛИЗИРУЕМОГО ЭЛЕМЕНТА}

Для определения значения массовой концентрации элемента методами ИХП отбирают пробу объекта окружающей среды и готовят жидкую пробу к анализу. Вначале выполняют измерение времени инверсии Фона $\left(t_{\Phi}\right)$, а затем Пробы $\left(t_{\Pi}\right)$. К жидкой пробе добавляют известную массу добавки стандартного образца ионов элемента $m$ и измеряют время инверсии пробы с Добавкой $\left(t_{д}\right)$. Согласно данной технологии возможно использование до 5 добавок стандартного образца ионов элемента. Время инверсии Фона $\left(t_{\Phi}\right)$, Пробы $\left(t_{\Pi}\right)$ и Добавки $\left(t_{д}\right)$ уточняют по линейному графику изменения массы ионов в растворе методом наименьших квадратов, что существенно при многократных измерениях Фона, Пробы и нескольких Добавках.

Зная отобранный объем пробы объекта ( $\left.V_{0}\right)$, общий объем раствора подготовленной пробы ( $\left.V_{1}\right)$, объем раствора пробы взятый для анализа $\left(V_{2}\right)$, 
определяют массовую концентрацию элемента $\rho$ (мг/дм $\left.{ }^{3}\right)$ в объекте окружающей среды по формуле:

$$
\rho=\frac{t_{\Pi}-t_{\Phi}}{t_{Д}-t_{\Pi}} \cdot m \cdot k
$$

где $k$ - переводной коэффициент, см$^{-3}$ (для расчета концентрации элемента в пробе объекта по значению его концентрации в электрохимической ячейке), который рассчитывается по формуле:

$$
k=\frac{V_{1}}{V_{2} \cdot V_{0}} .
$$

\section{ИСПОЛЬЗОВАНИЕ ОТДЕЛЬНЫХ ЭТАПОВ ИТ В АНАЛИЗАТОРАХ ТЯЖЕЛЫХ МЕТАЛЛОВ}

Одновременно с техническим воплощением отдельных этапов описанной ИТ в реальных системах измерения концентрации развивались общие подходы и формировался специализированный базовый пакет программ «ТCПО», обеспечивающий решение ряда задач обработки сигналов измерения: цифровой фильтрации данных [6], сглаживания, восстановления (интерполяции), аппроксимации и моделирования [7].

Так, был разработан целый ряд приборов определения концентрации тяжелых металлов: «Хронополярограф», «Анализатор ТМ», «МА-1020», «M-XА1000». Эти приборы не запоминали исходный сигнал инверсии в памяти, а обрабатывали его по мере поступления с автоматическим формированием сигнала интенсивности гистограммным способом. Они позволяли определять концентрацию 4 тяжелых металлов с чувствительностью 0,01 мкг/мл.

На основе пакета программ «ТС-ПО» был разработан и серийно выпускается анализатор солей тяжелых металлов «М-ХА1000-5». Этот анализатор выполняет измерение 5 тяжелых металлов (свинец, кадмий, цинк, медь и олово) с чувствительностью 0,001 мкг/мл, но без математического моделирования сигнала интенсивности. Более 300 этих приборов используются в аналитических лабораториях СЭС МЗ Украины и в других лабораториях, выполняющих экологический мониторинг окружающей среды.

\section{ПРИМЕНЕНИЕ РАЗРАБОТАННОЙ ИТ В ПРИБОРАХ ИЗМЕРЕНИЯ КОНЦЕНТРАЦИИ}

Накопленный опыт использования ИТ измерения концентрации токсичных элементов в реальных объектах дал возможность автору создать три принципиально новых электрохимических метода анализа и разработать конструкции приборов для их реализации, что значительно расширило возможности анализа базового метода ИХП:

- $\quad$ метод импульсной хронопотенциометрии (ИИХП), патент на конструкцию устройства стал победителем конкурса Украины «Винахід- 
2011» в химико-технологической отрасли [8];

- $\quad$ метод окислительной импульсной хронопотенциометрии (ОИХП)

[9];

- $\quad$ метод дифференциальной импульсной хронопотенциометрии (ДИХП) [10].

Техническую реализацию трех новых методов анализа ИИХП, ОИХП, ДИХП и базового метода ИХП объединено в одной конструкции прибора «Анализатор ИХП», который был разработан в Международном научноучебном центре информационных технологий и систем НАН Украины и МОН Украины.

Созданный анализатор позволяет определять содержание 14 токсичных элементов в растворе пробы в следующих диапазонах измерения массовой концентрации: ртути - от 0,0001 мкг/мл до 0,1 мкг/мл; мышьяка, свинца, кадмия, цинка, меди, никеля, кобальта — от 0,001 мкг/мл до 1,0 мкг/мл; олова, марганца, селена, йода, хрома, железа - от 0,01 мкг/мл до 1,0 мкг/мл. Для этих элементов Национальным университетом биоресурсов и природопользования Украины с участием автора были разработаны и утверждены четыре Методики выполнения измерения в питьевых, природных и сточных водах, а также в почве. «Анализатор ИХП» уже используется в научных лабораториях университетов и институтов.

Преобразование системы координат исходных данных, которое применяется в описанной ИТ, позволило разделить сигнал на отдельные компоненты и представить их в виде линейной суммы, а использование математических моделей для многокомпонентных растворов дало возможность устранить субъективный фактор при ручном определении границ диапазона отдельных компонентов в сигнале интенсивности, что увеличило точность и повторяемость измерений концентрации.

\section{ИСПОЛЬЗОВАНИЕ СОЗДАННОЙ ИТ В ДРУГИХ ТЕХНИЧЕСКИХ СИСТЕМАХ ИЗМЕРЕНИЙ}

Предложенная ИТ преобразования структуры исходных данных и моделирования компонентов сигнала интенсивности, а также базовый пакет программ «ТС-ПО» были успешно использованы для решения совершенно иной технической задачи - определения поосных нагрузок транспортного средства при взвешивании на платформенных весах [11]. Система поосного взвешивания на платформенных весах была впервые разработана автором и используется на Международном автомобильном пункте пропуска «Табаки - Мирное MD» Одесской таможни Украины.

\section{ВЫводЫ}

Разработанная ИТ измерения массовой концентрации токсичных элементов в жидких пробах методами инверсионной хронопотенциометрии, реализованная в приборе «Анализатор ИХП», позволила на порядок увеличить чувствительность измерения концентрации (до 0,0001 мкг/мл), 
значительно расширить число определяемых элементов (до 14) в разных объектах окружающей среды, повысить надежность и улучшить повторяемость результатов измерений концентрации.

Круг решаемых задач не ограничивается описанными техническими системами и может быть значительно расширен. Предложенная ИТ с использованием целенаправленного преобразования структуры исходных данных и моделирования компонентов измерения имеет универсальный характер и может быть применена для анализа различных сигналов физической или химической природы, в которых исходные значения монотонно возрастают или убывают.

1. Карнаухов О.І. Диференціальний варіант інверсійної хронопотенціометрії із заданим опором в окислювальному ланцюгу / О.І. Карнаухов, В.В. Гриневич, С.М. Скобець // Український хімічний журнал. - 1973. - № 39. - С. 710-714.

2. Карнаухов О.І. Теорія інверсійної хронопотенціометрії із заданим опором ланцюга / О.І. Карнаухов, В.М. Галімова, К.Р. Галімов // Науковий вісник НАУ. — 2000. № 32. - С. 204-209.

3. Устройство для предварительной обработки полярограмм: а. с. 1407241 СССР: МКИ A1 G01N27/26. / К.Р. Галимов, В.И. Лавриненко, Ю.Л. Серебрянников, Г.В. Цепков. № 4066909/31-25; Заявлено 11.05.1986; Опубл. 01.03.1988.

4. Васильев В.И. Индукция и редукция в проблемах экстраполяции / В.И. Васильев // Кибернетика и вычислительная техника. - 1998. — Вып. 116. - С. 65-81.

5. Васильев В.И. Практические аспекты теории редукции в задачах обнаружения и моделирования закономерностей / В.И. Васильев, И.В. Суровцев // Управляющие системы и машины. - 2001. - № 1. - С. 6-15.

6. Спосіб гістограмної цифрової фільтрації хронопотенціометричних даних: пат. 96367 Україна: МПК (2006) G01N 27/48. / I.В. Суровцев, В.М. Галімова, О.В. Бабак; Заявлено 11.05.2010; Опубл. 25.10.2011 // Промислова власність. — 2011. — № 20.

7. Суровцев И. В. Моделирование дифференциальных хронопотенциограмм суммой нормальных распределений / И.В. Суровцев, А.Э. Татаринов, С.К. Галимов. // Управляющие системы и машины. — 2009. - № 5. - С. 40-45.

8. Пристрій для вимірювання концентрації важких металів: пат. 96375 Україна: МПК (2006) G01N 27/48. / I.В. Суровцев, І.А. Мартинів, В.М. Галімова, О.В. Бабак; Заявлено 02.06.2010; Опубл. 25.10.2011 // Промислова власність. — 2011. — № 20.

9. Аналого-цифровий електрохімічний пристрій для вимірювання параметрів розчинів: пат. 104062 Україна: МПК (2006) G01N 27/48. / I.В. Суровцев, В.А. Копілевич, В.М. Галімова， І.А. Мартинов， О.В. Бабак; Заявлено 28.05.2012; Опубл. 25.12.2013 // Промислова власність. - 2013. — № 24.

10. Пристрій для вимірювання концентрації токсичних елементів: пат. 107412 Україна: МПК (2006) G01N 27/48. / I.В. Суровцев, С.К. Галімов, I.А. Мартинів, О.В. Бабак, В.М. Галімова; Заявлено 21.05.2013; Опубл. 25.12.2014 // Промислова власність. - 2014. — № 24.

11. Система поосьового зважування на платформних вагах: пат. 106013 Україна: МПК (2006) G01G 19/02. / I.В. Суровцев, О.В. Бабак, О.Е. Татарінов, Ю.А. Крижановський; Заявлено 06.08.2013; Опубл. 10.07 .2014 // Промислова власність. - 2014. — № 13. 


\section{TRANSFORMATION OF DATA STRUCTURE IN DETERMINING THE CONCENTRATION BY METHODS OF INVERSION CHRONOPOTENTIOMETRY}

\section{I.V. Surovtsev}

International Research and Training Center for Information Technologies and Systems of National Academy of Sciences of Ukraine and Ministry of Education and Science of Ukraine

Introduction. The complexity of direct measurement of the inversion time for the original signal in determining the concentration of toxic elements by inversion chronopotentiometry in the sample solution was not possible to determine its less than $0.1 \mathrm{mkg} / \mathrm{ml}$.

Purpose. Development of information technology for measurement the concentration of toxic elements in liquid tests of objects by methods of inversion chronopotentiometry, which give possibility essentially to increase sensitiveness and reliability in determination of the concentration.

Results. Using developed information technology determining the concentration of toxic elements in liquid tests of inversion chronopotentiometry in apparatus the analyzer allow to increase until 14 elements, to increase sensitiveness until $0,0001 \mathrm{mkg} / \mathrm{ml}$ and to improve repetition of measurements the concentration.

Conclusion. Information technology has a universal character and can be applied for the analysis of signals of different nature, where the values are monotonically increasing or decreasing.

Keywords: transformation of the data structure, methods of inversion chronopotentiometry, modeling, information technology.

1. Karnaukhov A.I., Grynevych V.V., Skobets E.M. Differential variant of inversion chronopotentiometry with a given resistance in oxidative circuit. Ukrainian chemical journal, 1973, no. 39, pp. 710-714 (in Ukrainian).

2. Karnaukhov A.I., Galimova V.M., Galimov K.R. Theory inversion chronopotentiometry with a given resistance of circuit. Scientific Visnyk of NAU, 2000, no. 32, pp. 204-209 (in Ukrainian).

3. Galimov K.R., Lavrynenko V.I., Serebryannikov J.L., Tsepkov G.V. The device for pretreating polarograms. Patent 1407241 USSR: Int.C1. G01N27/26, 1988 (in Russian).

4. Vasilyev V.I. Induction and reduction in problems of extrapolation. Cybernetics and Computer Engineering, 1998, no. 116, pp. 65-81(in Russian).

5. Vasilyev V.I., Surovtsev I.V. Practical aspects of the theory of reduction in problems of detection and modelling regularities. Control System and Computers, 2001, no. 1, pp.6-15 (in Russian).

6. Surovtsev I.V., Galimova V.M., Babak O.V. Method for histogram digital filtration of chrono-potentiometric data. Patent 96367 Ukraine, Int.C1. (2006) G01N 27/48, 2011 (in Ukrainian).

7. Surovtsev I.V., Tatarinov A.E., Galimov S.K. The modeling of the Differential Chronopotentiograms by the Sum of Normal Distributions. Control System and Computers, 2009, no. 5, pp.40-45 (in Russian).

8. Surovtsev I.V., Martynov I.A., Galimova V.M., Babak O.V. Device for measurement of concentration of heavy metals. Patent 96375 Ukraine, Int.C1. (2006) G01N 27/48, 2011 (in Ukrainian). 
9. Surovtsev I.V., Kopilevych V.A., Galimova V.M., Martynov I.A., Babak O.V. Analogdigital electro-chemical device for measurement of parameters of solutions. Patent 104062 Ukraine, Int.C1. (2006) G01N 27/48, 2013 (in Ukrainian).

10. Surovtsev I.V., Galimov S.K., Martynov I.A., Babak O.V., Galimova V.M. Device for measurement of concentration of toxic elements. Patent 107412 Ukraine, Int.C1. (2006) G01N 27/48, 2014 (in Ukrainian).

11. Surovtsev I.V., Babak O.V., Tatarinov O.E., Kryzhanovskyi Y.A. System for axle-by-axle weighing on platform scales. Patent 106013 Ukraine, Int.C1. (2006) G01G 19/02, 2014 (in Ukrainian).

Получено 03.03.2015 


\section{РЕШЕНИЕ ЗАДАЧИ ОПТИМАЛЬНОГО ВЫБОРА С ГРУППОВЫМ ПРОСМОТРОМ С ПОМОЩЬЮ ТЕОРЕТИКО-ИГРОВОГО ПОДХОДА}

\section{С.И. Доценко}

\section{Киевский национальный университет имени Тараса Шевченко}

Рассмотрены три варианта игровой задачи выбора наилучшего элемента для случая, когда элементы разбиты на группы и за один шаг осуществляется одновременный просмотр элементов всей группы. Исходя из концепции сложного рационального поведения, для каждого из случаев найдены равновесные ситуации игры.

Ключевые слова: задача оптимального выбора, пороговая стратегия, групповой просмотр, равновесие Байеса-Нэша, цена анархии.

Розглянуто три варіанти ігрової задачі оптимального вибору для випадку, коли об'єкти розбито на групи та за один крок здійснюється одночасний перегляд всіх елементів групи. Виходячи 3 концепції складної раціональної поведінки, для кожного 3 випадків знайдено ситуації рівноваги у грі.

Ключові слова: задача оптимального вибору, порогова стратегія, груповий перегляд, рівновага Байєса-Неша, ціна анархії.

\section{ВВЕДЕНИЕ}

Задача оптимального выбора (известная также как задача секретаря) является одной из классических задач теории вероятностей и служит иллюстративным примером таких разделов математики, как оптимальная остановка марковских процессов, динамическое программирование, принятие решения в условиях риска и неопределенности. Рассмотрено обобщение задачи оптимального выбора на случай, когда просмотр осуществляется одновременно целыми группами. Для такой задачи группового просмотра рассмотрена конфликтная ситуация, когда в поиске наилучшего элемента задействованы два агента с различными интересами. Данная конфликтная ситуация описывается при помощи аппарата теории игр. Рассмотрены три модели взаимодействия агентов, которые могут возникать в ходе поиска. Для каждого из случаев найдены точки равновесия по Нэшу и вычислена цена анархии.

Целью данной статьи является построение иллюстративных примеров, демонстрирующих эффект анархии в игровой задаче оптимального выбора с групповым просмотром, в зависимости от степени информированности игроков.

\section{ПОСТАНОВКА ИГРОВОЙ ЗАДАЧИ ГРУППОВОГО ПРОСМОТРА}

Вначале вкратце напомним классическую постановку задачи оптимального выбора. Пусть есть $n$ объектов, упорядоченных по качеству. Пусть некто знакомится с данными объектами в случайном порядке (т.е. это означает, что все $n$ ! перестановок объектов, задающих порядок, в котором они могут встретиться просматривающему, равновероятны). При просмотре 
каждого из объектов нужно принять решение - остановить просмотр на данном объекте в надежде, что он окажется наилучшим среди всех, либо отвергнуть его и продолжить просмотр. Возвращаться к ранее просмотренным (и отвергнутым) объектам нельзя.

В [1] было рассмотрено обобщение задачи оптимального выбора на случай, когда объекты разбиты на группы и осуществляется одновременный просмотр кандидатов в каждой группе. После просмотра кандидатов группы аналогично классической задаче в случае, если в группе присутствует наилучший кандидат среди всех ранее просмотренных элементов (такой элемент принято называть максимальным), нужно принять решение выбрать этого кандидата и закончить просмотр либо же отвергнуть его и продолжить просмотр - возвращаться к ранее отвергнутым кандидатам нельзя.

Рассмотрим три варианта игры, которая базируется на задаче оптимального выбора с групповым просмотром, в которой нужно сделать удачный выбор не позже противника. Все три варианта игры имеют общие правила, приведенные ниже, и различаются только степенью информированности игроков.

\section{ПРАВИЛА ИГРЫ}

1) В игре принимают участие два игрока.

2) Каждый игрок осуществляет выбор на своем множестве элементов.

3) В начале игры множество элементов каждого из игроков случайно (с равномерным распределением и независимо) делится на две группы.

4) Каждый из игроков осуществляет поиск максимального элемента, после чего одному или двум игрокам выплачивается выигрыш, который начисляется следующим образом:

4.1. Если удачный выбор сделал один из игроков (а другой - нет), то ему достается 1.

4.2. Ничья. Если удачный выбор сделали оба игрока, и номера групп совпадают (оба в 1-й либо оба во 2-й группе), то выигрыш делится между ними поровну и каждый получает $1 / 2$.

4.3. Приоритет раннего выбора. Если удачный выбор сделали оба игрока, один в 1-й, другой во 2-й группе, то тому, кто сделал удачный выбор в 1-й группе достается 1, другой же не получает ничего.

4.4. Обоюдный проигрыш. Если оба игрока не сделали удачного выбора, то никто не получает ничего.

Следует заметить, что в то время, как задача оптимального выбора с групповым просмотром для случая двух групп является тривиальной (напомним, что в этом случае следует остановиться на максимальном элементе большей из двух групп независимо от порядка их следования), то игровая задача даже для такого простого случая не столь очевидна.

Аналогичная постановка игры для классической задачи оптимального выбора рассматривалась в [2]. Там было доказано, что оптимальные стратегии игроков, целью которых является найти наилучший элемент и при этом сделать остановку раньше противника, имеют пороговый вид - 
пропускать все объекты с индексами, меньшими $z^{*} N$, где $z^{*}-$ корень уравнения $-\ln (z)-z \cdot \ln ^{2}\left(\frac{z}{2}\right)=1, \quad$ примерно равный 0.295 . Пороговое значение по сравнению с классической задачей $\frac{1}{e} \approx 0.368$ смещено влево, что можно объяснить «эффектом спешки». Ошибки неверного определения наилучшего элемента при этом возрастают, но это необходимо для того, чтобы опередить противника.

Рассмотренные ниже три примера игровой модификации поиска наилучшего элемента в задаче с групповым просмотром наглядно иллюстрируют вариативность поведения игроков в зависимости от степени их информированности.

\section{ПЕРВЫЙ ВАРИАНТ — СИММЕТРИЧНАЯ ИГРА С НЕПОЛНОЙ ИНФОРМАЦИЕЙ}

Пусть множества элементов игроков были разделены на две группы, и при этом каждый из игроков знает, каким образом произошло деление элементов его группы, и не знает, как были разделены элементы противника.

Оказывается, что в этой ситуации игрокам следует применять пороговые стратегии, которые заключаются в том, чтобы выбирать элемент из первой группы при условии, что доля этой группы от общего числа элементов составляет не менее заданного порога $u$. Согласно формуле полной вероятности, каждая такая пороговая стратегия порождает некоторое вероятностное распределение исходов игрока:

$$
\left(p_{1}(u), p_{2}(u), 1-p_{1}(u)-p_{2}(u)\right)=\left(\frac{1}{2}-\frac{u^{2}}{2}, u-\frac{u^{2}}{2}, u^{2}-u+\frac{1}{2}\right),
$$

где первая компонента вектора описывает вероятность того, что был осуществлен удачный выбор из первой группы, вторая - удачный выбор из второй, а третья - это вероятность того, что наилучший элемент не был выбран.

Назовем распределение исходов $\left(p_{1}^{\prime}, p_{2}^{\prime}\right)$ неэффективным, если существует другое распределение исходов $\left(p_{1}, p_{2}\right)$, порожденное некоторой другой стратегией выбора, такое, что $p_{1} \geq p_{1}^{\prime}, p_{1}+p_{2} \geq p_{1}^{\prime}+p_{2}^{\prime}$, и одно из двух неравенств является строгим.

Поскольку более эффективное распределение исходов является лучшим ответом на любую стратегию противника, то стратегии, порождающие неэффективные исходы заведомо не являются эффективными.

Следует заметить, что все распределения исходов, порожденные не пороговыми стратегиями, являются неэффективными (доказательство этого факта простое, но громоздкое, поэтому не включено в данную статью). Поэтому равновесное состояние следует искать в классе пороговых стратегий. Поскольку распределение исходов выбора каждого из игроков случайно, то искомое равновесие является равновесием Байеса-Нэша.

(C) С.И. Доценко, 2015 
Пусть оба игрока применяют пороговые стратеги, первый - с порогом $x$, второй - с порогом $y$; тогда, согласно приведенных правил игры, выигрыш первого игрока составит:

$W_{1}(x, y)=p_{1}(x)\left(1-p_{1}(y)\right)+p_{2}(x)\left(1-p_{1}(y)-p_{2}(y)\right)+\frac{1}{2} p_{1}(x) p_{1}(y)+\frac{1}{2} p_{2}(x) p_{2}(y)$.

Подставляя (1) в (2), и группируя подобные по степеням $x$, имеем:

$$
W_{1}(x, y)=\frac{1}{8}\left[\left(-4 y^{2}+2 x-5\right) x^{2}+\left(6 y^{2}-4 y+4\right) x+\left(y^{2}+3\right)\right] .
$$

Наилучший выбор значения порога первым игроком в ответ на выбор второго игрока составляет:

$$
x_{0}=B R(y)=\frac{3 x^{2}-2 x+2}{4 x^{2}-2 x+5} .
$$

Равновесие Байеса-Нэша находится из системы $\left\{\begin{array}{l}x=B R(y) \\ y=B R(x)\end{array}\right.$.

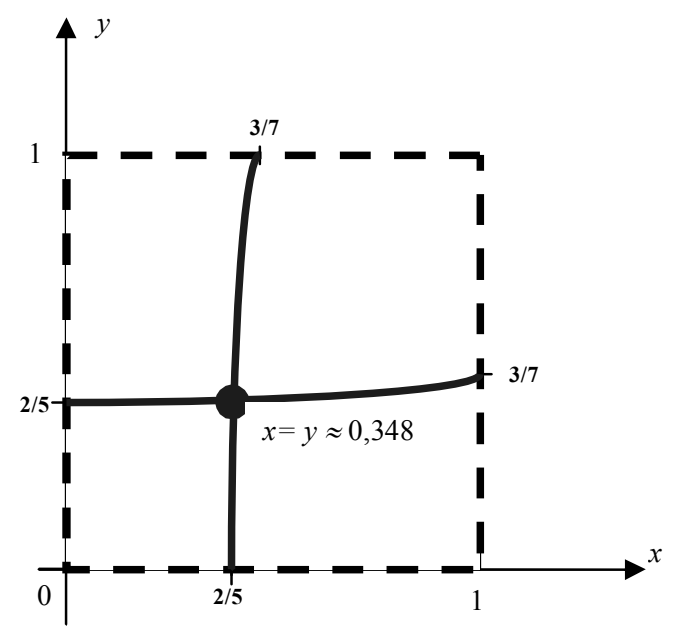

Рис. 1. Точка равновесия Нэша

Оказывается, что решение этой системы единственно и находится из соотношения $x=\mathrm{BR}(x), y=x$, что, в свою очередь, сводится к решению кубического уравнения $4 x^{3}-5 x^{2}+7 x-2=0$. Это уравнение на интервале [0;1] имеет единственный корень, примерно равный 0.348 (Рис. 1), при этом выигрыши игроков примерно равны 0.463 .

Для сравнения, рассмотрим ситуацию корпоративного поведения игроков. В этом случае каждому из участников игры безразлично, кому именно достанется выигрыш - ему или другому игроку. Игроки должны минимизировать вероятность ситуации, в которой выигрыш не достанется никому. Вероятность такого события равна:

$$
p_{3}(x) p_{3}(y)=\left(x^{2}-x+\frac{1}{2}\right)\left(y^{2}-y+\frac{1}{2}\right)
$$


Данное выражение достигает минимума, равного $\frac{1}{16}$, при $x=y=\frac{1}{2}$. Значит, оба игрока должны применять пороговые стратегии со значением порога, равным $\frac{1}{2}$, тогда их суммарный выигрыш составит $\frac{15}{16} \approx 0.938$.

Таким образом, цена анархии равна $\frac{0.938}{2 \cdot 0.463} \approx 1.013$.

\section{ВТОРОЙ ВАРИАНТ ИГРЫ - АСИММЕТРИЧНАЯ ИНФОРМИРОВАННОСТЬ}

Пусть, как и в предыдущем варианте игры, игроки знают, как разделено их множество элементов, и не знают, как разделено множество элементов противника. Пусть второй игрок применяет какую-либо пороговую стратегию и исход его выбора (т. е. удачный выбор в 1-й группе, удачный выбор во 2-й группе либо неудачный выбор) становится известным первому игроку. Используя эту информацию, первый игрок выбирает пороговую стратегию, наиболее выгодную для себя. После этого исходы игроков сравниваются, и происходит выплата выигрыша согласно приведенным правилам.

Рассмотрим вспомогательный пример. Пусть принимающему решение игроку за удачный выбор в 1-й группе платят 1, а за удачный выбор во 2-й $0 \leq \alpha \leq 1$. Тогда он должен выбирать значение порога, максимизирующее ожидаемую выплату:

$$
p_{1}(u)+\alpha \cdot p_{2}(u) \rightarrow \max .
$$

Пусть исход выбора 2-го игрока - 1 (удачный выбор в 1-й группе). Тогда, узнав об этом, 1-й игрок решает задачу (4) с $\alpha=0$ (удачный выбор во 2-й группе для него не имеет никакой ценности). В этом случае он полагает $x=0$, и ожидаемые выигрыши игроков составляют $V_{2}=1 / 4, V_{1}=3 / 4$.

Пусть исход выбора 2-го игрока - 2 (удачный выбор во 2-й гр.). Тогда, узнав об этом, 1-й игрок решает задачу (4) с $\alpha=1 / 2$ (при исходе 1 он заберет выигрыш целиком, а при исходе 2 - разделит поровну со 2-м игроком). В этом случае он полагает $x=1 / 3$, и ожидаемые выигрыши игроков составляют $V_{1}=7 / 12, V_{2}=5 / 12$.

Пусть исход выбора 2-го игрока - 3 (неудача). Тогда, узнав об этом, 1-й игрок решает задачу (4) с $\alpha=1$ (его одинаково устраивает как 1-й, так и 2-й исход). В этом случае он полагает $x=1 / 2$, и ожидаемые выигрыши игроков составляют $V_{1}=3 / 4, V_{2}=0$.

Таким образом, второй игрок, полагая, что первый, узнав о его выборе, будет действовать оптимальным для себя образом, решает задачу максимизации своего ожидаемого выигрыша:

$$
\frac{3}{4} p_{1}(y)+\frac{5}{12} p_{2}(y)=\frac{1}{24}\left(-14 y^{2}+10 y+9\right) \rightarrow \max .
$$


Данное выражение достигает максимума при $y_{0}=\frac{5}{14}$, тогда распределение исходов 2-го игрока составляет

$$
\left(p_{1}\left(\frac{5}{14}\right), p_{2}\left(\frac{5}{14}\right), p_{3}\left(\frac{5}{14}\right)\right) \approx(0.436,0.293,0.271) .
$$

Тогда ожидаемый выигрыш 2-го игрока составит

$$
0.436 \cdot \frac{3}{4}+0.293 \cdot \frac{5}{12} \approx 0.449,
$$

а ожидаемый выигрыш 1-го

$$
0.436 \cdot \frac{1}{4}+0.293 \cdot \frac{7}{12}+0.271 \cdot \frac{3}{4} \approx 0.483
$$

Выигрыш 1-го игрока, как и следовало ожидать, несколько больше, чем 2-го, поскольку он более информирован. При этом суммарный выигрыш игроков составляет 0.932 , а цена анархии $-\frac{0.938}{0.932} \approx 1.006$.

\section{ТРЕТИЙ ВАРИАНТ ИГРЫ - ПОЛНАЯ СИММЕТРИЧНАЯ ИНФОРМИРОВАННОСТЬ}

Пусть теперь каждый из игроков знает, как разделено на группы множество его элементов и множество элементов противника. Сложившуюся ситуацию будем описывать парой $(x, y)$, где $x$ и $y$ - доли элементов первой группы для 1-го и 2-го игроков, соответственно. Проанализировав данную информацию, они независимо (и в тайне друг от друга) принимают решение о стратегии выбора. В данном случае стратегия каждого из игроков состоит из двух возможных альтернатив - из какой из двух групп выбирать максимальный элемент. Поэтому профиль стратегий игроков можно описать в виде $\operatorname{str}(i, j)$, где $(i, j) \in\{0 ; 1\}$, а игра сводится к биматричной с такими матрицами:

$$
A=\left(\begin{array}{cc}
x\left(1-\frac{y}{2}\right) & x \\
(1-x)(1-y) & \frac{(1-x)(1+y)}{2}
\end{array}\right), \quad B=\left(\begin{array}{cc}
y\left(1-\frac{x}{2}\right) & (1-y)(1-x) \\
y & \frac{(1-y)(1+x)}{2}
\end{array}\right) .
$$

Профиль стратегий $\operatorname{str}(1,1)$ является равновесным по Нэшу, если выполнены условия $A_{11}(x, y) \geq A_{21}(x, y), \quad B_{11}(x, y) \geq B_{21}(x, y)$, которые сводятся к системе неравенств

$$
\left\{\begin{array}{l}
\left(x-\frac{2}{3}\right)\left(y-\frac{4}{3}\right) \leq \frac{2}{9} \\
\left(x-\frac{4}{3}\right)\left(y-\frac{2}{3}\right) \leq \frac{2}{9} .
\end{array}\right.
$$

Профиль стратегий $\operatorname{str}(2,2)$ является равновесным по Нэшу, если выполнены условия, $A_{22}(x, y) \geq A_{12}(x, y), \quad B_{22}(x, y) \geq B_{21}(x, y)$, которые 
сводятся к системе неравенств

$$
\left\{\begin{array}{l}
y \leq 1-\frac{2}{x+3} \\
x \leq 1-\frac{2}{y+3}
\end{array}\right.
$$

Области равновесий по Нэшу, соответствующие профилям стратегий $\operatorname{str}(1,1)$ и $\operatorname{str}(2,2)$, показаны на рис. 2 и рис. 3 соответственно.

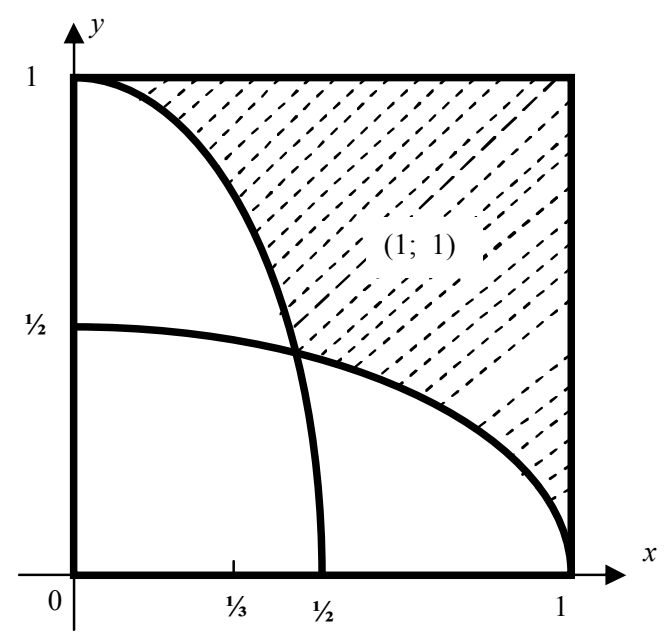

Рис. 2. Область равновесия для профиля $\operatorname{str}(1,1)$

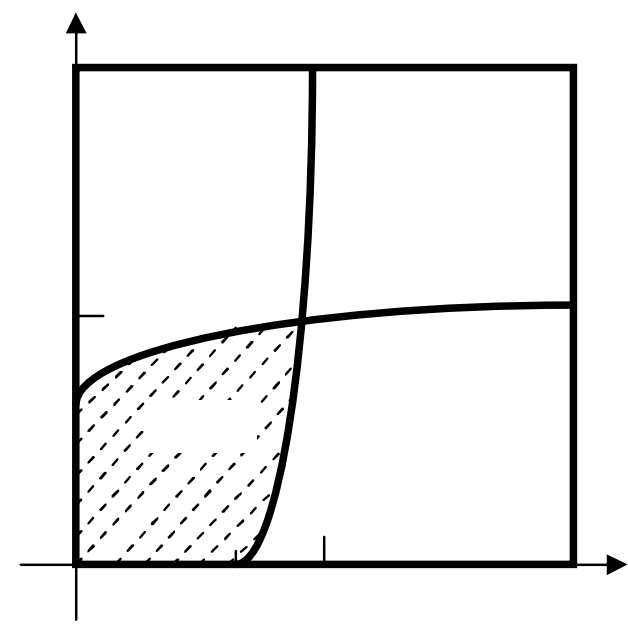

Рис. 3. Область равновесия для профиля $\operatorname{str}(2,2)$

Ситуация $(1,2)$ является равновесной по Нэшу, если выполнены условия, $A_{12}(x, y) \geq A_{22}(x, y), \quad B_{12}(x, y) \geq B_{11}(x, y)$, которые сводятся к системе неравенств 


$$
\left\{\begin{array}{c}
\left(x-\frac{4}{3}\right)\left(y-\frac{2}{3}\right) \geq \frac{2}{9}, \\
x \geq 1-\frac{2}{y+3} .
\end{array}\right.
$$

Поскольку игра симметричная, то область, в которой ситуация $(2,1)$ является равновесной по Нэшу, имеет вид

$$
\left\{\begin{array}{c}
\left(x-\frac{2}{3}\right)\left(y-\frac{4}{3}\right) \geq \frac{2}{9}, \\
y \geq 1-\frac{2}{x+3} .
\end{array}\right.
$$

Области равновесий по Нэшу, соответствующие профилям стратегий $\operatorname{str}(1,2)$ и $\operatorname{str}(2,1)$, показаны на рис. 4 и рис. 5 соответственно.

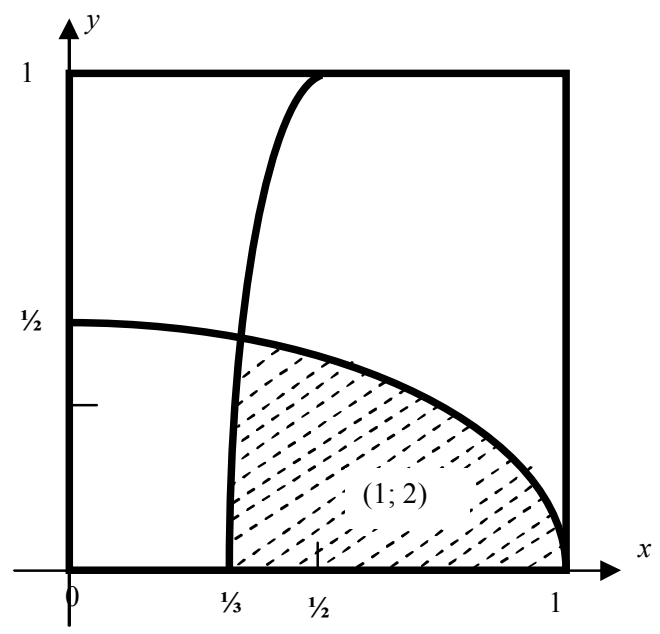

Рис. 4. Область равновесий для профиля $\operatorname{str}(1,2)$

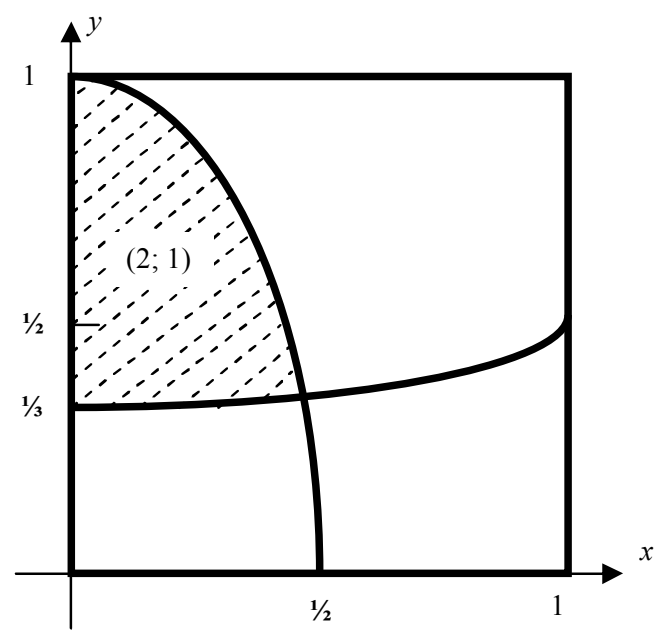

Рис. 5. Область равновесий для профиля $\operatorname{str}(2,1)$

(C) С.И. Доценко, 2015

22

ISSN 0452-9910. Кибернетика и вычисл. техника. 2015. Вып. 180 
Области равновесия для профилей стратегий $\operatorname{str}(1,2)$ и $\operatorname{str}(2,1)$ имеют общую часть. Например, при $x=0.416, y=0.416$ имеет место биматричная игра с такими платежными матрицами:

$$
A=\left(\begin{array}{ll}
0.329 & 0.416 \\
0.341 & 0.413
\end{array}\right), \quad B=\left(\begin{array}{ll}
0.329 & 0.321 \\
0.416 & 0.413
\end{array}\right) .
$$

Данная игра имеет две точки равновесия $(1,2)$ и $(2,1)$, в чем легко убедиться непосредственно.

\section{Выводы}

Найденные ситуации равновесия Байеса-Нэша в задаче поиска наилучшего элемента с возможностью группового просмотра показывают, что в зависимости от постановки задачи вид равновесия может принимать различные формы - как точечное равновесие (примеры 1 и 2), так и семейство равновесий, описываемых замкнутой геометрической областью (пример 3), причем в первых двух случаях имеет место незначительный эффект анархии.

1. Bruss T. Sum the odds to one and stop / T. Bruss // The annals of probability. -2000 . Vol. 28, No. 3. - P 1384-1391.

2. Мазалов В. Математическая теория игр и ее приложения / В. Мазалов. - СПб.: Изд-во «Лань», 2010. - 446 с.

\section{UDC 519.83}

\section{SOLUTION OF THE PROBLEM OF OPTIMAL CHOICES WITH A GROUP BROWSING BY A GAME-THEORETIC APPROACH}

\section{S.I. Dotsenko \\ Taras Shevchenko National University of Kyiv}

Introduction. The problem of optimal choice in the case when the objects are divided into groups and carried out the simultaneous viewing of candidates in each group was considered by Bruss T. If watching the group of candidates is similar to the classical problem and the group is presented by the best candidate among all previously viewed items (such an element is called a maximum) to make a decision - choose this candidate and finish viewing or reject it and continue - the returning to the previously rejected candidates prohibited.

In this case, the optimal rule for selecting the best candidate based on the so-called «choice theorem» (or «Bruss theorem»).

For the particular case of two groups the search strategy is trivial - namely to ignore the smaller group and to view the bigger one. However, if this case is considered as two person game, the problem appeared to be intriguing.

The purpose of the article is to find Nash equilibrium for two persons game, associated to group search secretary problem at the following set of rules.

1) Each player makes his choice at his own set of elements.

2) At the beginning the set of searched elements are divided at random into two subsets according to uniform distribution.

(C) С.И. Доценко, 2015 
3) Each of two players searches the best element (i.e. solve the secretary problem for two groups). After that, the prize is paid to one or two players according to the following rules. If one of the players made his lucky choice and the other one not, then the first one got 1 . If the both players made their lucky choice at the same group then they share the price and got $1 / 2$ each. If one of the players got his lucky choice at the first group, and another one at the second group, then the first one got 1, and the second one got nothing.

Results. For the considered game the Bayes-Nash equilibrium is obtained for three different cases. Equilibrium points are shown at two-dimensional diagram. Depending on the problem statement, Nash equilibrium area may take different shapes - either single point (cases 1 and 2) or family of points inside the closed curve (case 3). In first two cases, the slight effect of anarchy is observed.

Conclusion. The results confirm the general principle, that the game situation solutions, based on even trivial optimization problems, makes this solutions to be complicated. Based on the concept of a complex rational behavior for each of the cases found equilibrium situations of the game.

Keywords: problem of optimal choice,threshold strategy, group search, BayesNash equilibrium, the price of anarchy.

1. Thomas Bruss. Sum the odds to one and stop. The annals of probability, 2000, vol. 28, no. 3 , pp. 1384-1391.

2. V. Mazalov. Mathematical theory of games and it's applications. Saint Peterburg: Lan, 2010, 446 p. (in Russian).

Получено 30.03.2015 


\title{
ТЕХНОЛОГИЯ РЕШЕНИЯ ЗАДАЧИ МОДЕЛИРОВАНИЯ И ПРОГНОЗИРОВАНИЯ НА ОСНОВЕ ИНДУКТИВНОГО ПОДХОДА
}

\section{Е.А. Савченко}

\begin{abstract}
Международный научно-учебный центр информационных технологий и систем НАН Украины и МОН Украины
\end{abstract}

Разработана и описана технология решения задачи моделирования и прогнозирования на основе индуктивного подхода. В ее основе лежит комбинаторный алгоритм МГУА и метод доопределения. Технология позволяет автоматически, по выборке экспериментальных данных, находить модель объекта или процесса с использованием двух внешних критериев выбора: точности и несмещенности. Применение технологии в реальных задачах моделирования и прогнозирования подтвердило ее эффективность.

Ключевые слова: индуктивный подход, комбинаторный алгоритм метода группового учета аргументов, моделирование, прогнозирование, технология.

Розроблено та описано технологію розв'язання задачі моделювання та прогнозування на основі індуктивного підходу. В iii основі лежить комбінаторний алгоритм МГУА та метод довизначення. Технологія дозволяє автоматично, за вибіркою експериментальних даних, знаходити модель об'єкту або процесу з використанням двох зовнішніх критеріїв вибору: точності і незміщеності. Застосування технології в реальних задачах моделювання та прогнозування підтвердило iï ефективність.

Ключові слова: індуктивний підхід, комбінаторний алгоритм методу групового урахування аргументів, моделювання, прогнозування, технологія.

\section{ВВЕДЕНИЕ}

Задача построения моделей, которые описывают или прогнозируют поведение наблюдаемого объекта или процесса, является неотъемлемой составной частью решения задач управления и принятия решений. Для построения математической модели в общем случае следует определить ее структуру и оценить параметры, то есть решить задачу структурнопараметрической идентификации или моделирования по выборке статистических или экспериментальных данных.

Часто реальные прикладные задачи характеризуются нестабильностью измерений, трудно учесть все факторы, влияющие на процесс. Для моделирования и прогнозирования таких данных целесообразно использовать индуктивный подход как метод построения моделей по данным наблюдений. Индуктивные методы позволяют автоматически находить зависимости, скрытые в выборке экспериментальных данных.

Один из основных алгоритмов индуктивного моделирования комбинаторный алгоритм метода группового учета аргументов (МГУА). Однако применение этого алгоритма в реальных задачах показало, что не всегда удается однозначно определить модель по одному заданному критерию. Для такого случая разработан метод доопределения модели на 
основе комбинаторного алгоритма МГУА.

Цель работы - разработать технологию решения задачи моделирования и прогнозирования по выборке экспериментальных данных на основе комбинаторного алгоритма МГУА и метода доопределения, которая позволяла бы строить оптимальную модель в реальных прикладных задачах.

\section{ОСОБЕННОСТИ ИНДУКТИВНОГО ПОДХОДА}

Индуктивное моделирование базируется на переборе множества моделей-кандидатов по внешним критериям селекции и решает задачу структурно-параметрической идентификации. Одним из наиболее эффективных методов моделирования по экспериментальным данным в условиях неполноты информации является метод группового учета аргументов, автором которого является академик А.Г. Ивахненко [1-2].

В отличие от регрессионного анализа, где структура модели задается, в МГУА структура оптимальной модели и ее параметры находятся посредством самоорганизации моделей, то есть испытания многих моделейкандидатов по внешним критериям селекции. В [3] проводилось сравнение эффективности применения регрессионного анализа и МГУА для прогнозирования экономических процессов, где сравнивались прогнозирующие свойства моделей, построенных на основе метода наименьших квадратов (МНК) и МГУА, на примерах анализа объема производства легкой промышленности и инфляции. Было показано, что прогнозирующие свойства модели МГУА значительно выше, причем модель оптимальной сложности значительно проще, чем полная модель, построенная по МНК. Некоторые факторы, которые не вошли в модель, построенную по МГУА, были не просто лишними и малоинформативными, а также «вредными» в условиях имеющейся короткой выборки данных.

МГУА построен на принципах самоорганизации, а именно, на основе следующих двух принципов.

1. При постепенном усложнении структуры значения внешних критериев сначала уменьшаются, а затем увеличиваются, то есть в их зависимости от сложности моделей существует минимум; только внешние критерии, рассчитанные по данным, которые не использовались для построения моделей, имеют такой минимум. Поэтому в МГУА применяется деление не менее чем на две независимые выборки данных [1]. Одна из них используется для оценки параметров, а вторая - для оценки качества модели. Применять только один критерий принципиально нельзя, поскольку может возникнуть ситуация: «чем сложнее модель, тем она точнее». Все критерии, которые используются в МГУА, основаны на разбиении выборки, т.е. на «внешней» информации, поэтому названы внешними критериями.

2. Особенностью МГУА является обеспечение «свободы выбора». При постепенном усложнении структуры моделей на каждом ряду из лучших по внешним критериям моделей отбирается не одна, а несколько лучших моделей [2]. Наиболее известным среди методов индуктивного моделирования является комбинаторный алгоритм МГУА [4]. Это метод, 
который не требует доказательства сходимости, поскольку выполняет полный перебор всех возможных моделей-кандидатов. МГУА разработан для решения задач моделирования сложных систем, прогнозирования, идентификации и аппроксимации многофакторных систем, диагностики, распознавания образов и кластеризации данных.

\section{ПОСТРОЕНИЕ ТЕХНОЛОГИИ МОДЕЛИРОВАНИЯ И ПРОГНОЗИРОВАНИЯ НА ОСНОВЕ ИНДУКТИВНОГО ПОДХОДА}

Применение комбинаторного алгоритма МГУА в прикладных задачах показало, что возникает необходимость в повышении эффективности выбора оптимальной модели, поскольку часто вместо оптимальной модели избирается множество моделей-кандидатов с близкими значениями внешнего критерия регулярности. Поэтому на основе комбинаторного алгоритма МГУА был разработан метод поиска оптимальной модели - метод доопределения с применением критерия несмещенности [5].

Это двухкритериальный метод доопределения с использованием критерия несмещенности ошибок, который позволяет устранить неоднозначность выбора модели в реальных задачах моделирования за счет применения целесообразной последовательности критериев селекции. Исследование различных последовательностей внешних критериев МГУА показало, что следующая последовательность является целесообразной [6]: сначала применяется критерий точности, а затем критерий несмещенности. Такое последовательное применение двух внешних критериев повышает помехоустойчивость модели.

Индуктивное моделирование при самоорганизации моделей выдвигает определенные требования к выборке исходных данных. Во-первых, выборка должна содержать те аргументы, которые влияют на исходную переменную. Способы выбора информативных аргументов приведены в [7]. Во-вторых, выборка должна быть полной, то есть не содержать пропущенных значений [8]. Если данные в выборке имеют разную размерность, желательно их нормировать.

Разработана компьютерная технология моделирования и прогнозирования, которая включает следующие этапы [9]:

1) предварительная обработка выборки данных;

2) построение модели идентификации или прогноза.

На рис. 1 показана общая схема компьютерной технологии моделирования и прогнозирования.

Выборка данных (1) подается на вход блока предварительной обработки данных (2), после чего поступает на вход комбинаторного алгоритма МГУА (3), на выходе которого получаем оптимальную модель (4) или множество моделей-кандидатов, которые имеют минимальное значение внешнего критерия. В случае необходимости при построении модели осуществляется доопределение выбора модели с целью отбора оптимальной модели.

Блок 1 - Исходная выборка данных представляет собой матрицу экспериментальных данных, содержащую $n$ наблюдений за ходом процесса 
(строк выборки), $m$ входных переменных (столбцов выборки) и вектор $n$ наблюдений выходной переменной.

Блок 2 - Предварительная обработка выборки данных.

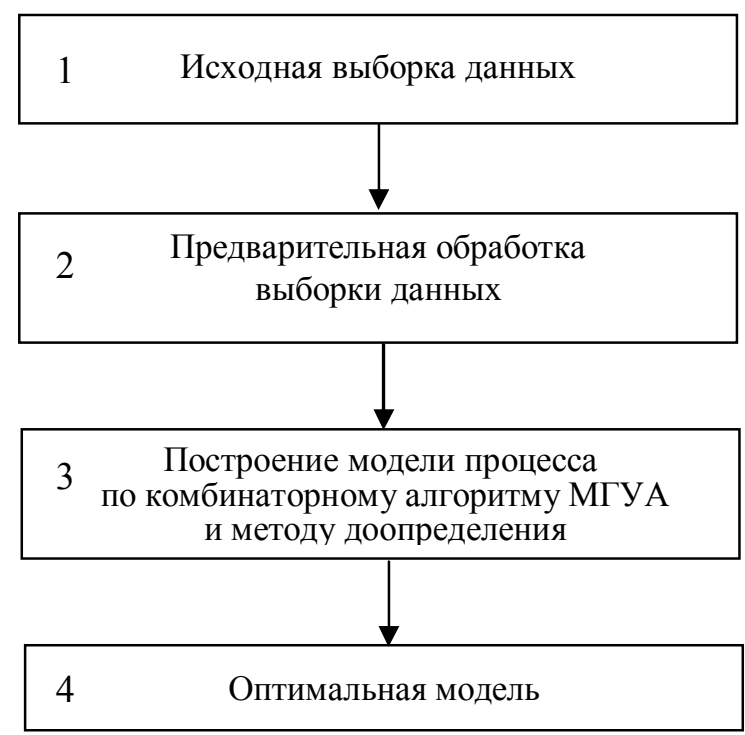

Рис. 1. Построение прогнозирующей модели с использованием комбинаторного алгоритма МГУА и метода доопределения

Предварительная обработка выборки данных включает следующие задачи [8]:

- восстановление пропущенных данных наблюдений и расширение состава аргументов за счет дополнительных;

- нормирование исходных данных;

- расширение выборки данных за счет введения дополнительных аргументов;

— выбор наиболее информативных аргументов;

- оптимизация размера выборки за счет поиска аналогов в предыстории.

Рассмотрим этапы этого блока подробнее.

1. Сначала выборка проверяется на наличие пропущенных значений. Для восстановления пропущенных данных в выборке данных применяется комбинаторный алгоритм МГУА с различными шаблонами считывания данных [9]. По заданному шаблону формируется расширенная выборка данных, по которой определяется лучшая модель и рассчитывается значение пропущенного элемента. Для каждого пропуска в данных рассчитывается новая модель.

2. Следующим шагом является нормирование данных, которое целесообразно применять только тогда, когда данные имеют разную размерность или очень разные масштабы чисел.

3. Далее формируются дополнительные аргументы. Расширение пространства моделирования за счет введения таких аргументов может повысить точность полученной модели. К данным исходной выборки 
добавляются попарные произведения начальных переменных, запаздывания и тому подобное [7].

4. Оценка информативности входных и сформированных переменных осуществляется с помощью расчета значений модуля коэффициента корреляции каждой переменной с выходной переменной. Переменные, которые имеют малое значение модуля, могут быть исключены из множества входных аргументов.

5. Для оптимизации размера выборки рассчитывается значение евклидовых расстояний вектор-строк таблицы данных, а затем отбирается из всего множества наблюдений необходимое количество ближайших к исходному наблюдению.

Блок 3 - Построение модели процесса по комбинаторному алгоритму МГУА и методу доопределения.

Рассмотрим подробнее структуру комбинаторного алгоритма МГУА с доопределением по критерию несмещенности ошибок. Сначала все наблюдения ранжируются по значениям дисперсии:

$$
D_{j}^{2}=\sum_{i=1}^{m}\left(x_{i j}-\bar{x}_{i}\right)^{2},
$$

где $j=1,2, \ldots, m, m$ - число аргументов,

$$
\bar{x}_{i}=\frac{1}{n} \sum_{j=1}^{n} x_{i j},
$$

где $n$ - общее число точек.

Для определения оптимальной модели выборки должны как можно больше отличаться [1]. Поэтому исходная выборка делится на две части: первые из всех наблюдений, которые имеют наибольшее значение дисперсии, составляют обучающую подвыборку, оставшаяся часть всех наблюдений проверочную подвыборку (с наименьшим значением дисперсии). Для всех генерируемых в процессе работы алгоритма моделей различной сложности рассчитываются значения критерия регулярности. По минимуму этого критерия выбирается множество лучших моделей, для каждой модели рассчитывается значение критерия несмещенности ошибок и по его минимуму выбирается оптимальная модель (Блок 4).

На рис. 2 показана процедура доопределениюя по критерию несмещенности ошибок.

На основе разработанной технологии разработан комплекс программ, который позволяет решать задачи предварительной обработки выборки данных для моделирования и прогнозирования. Комплекс программ, ядром которого является комбинаторный алгоритм МГУА, реализован на языке программирования $\mathrm{C}++$. 


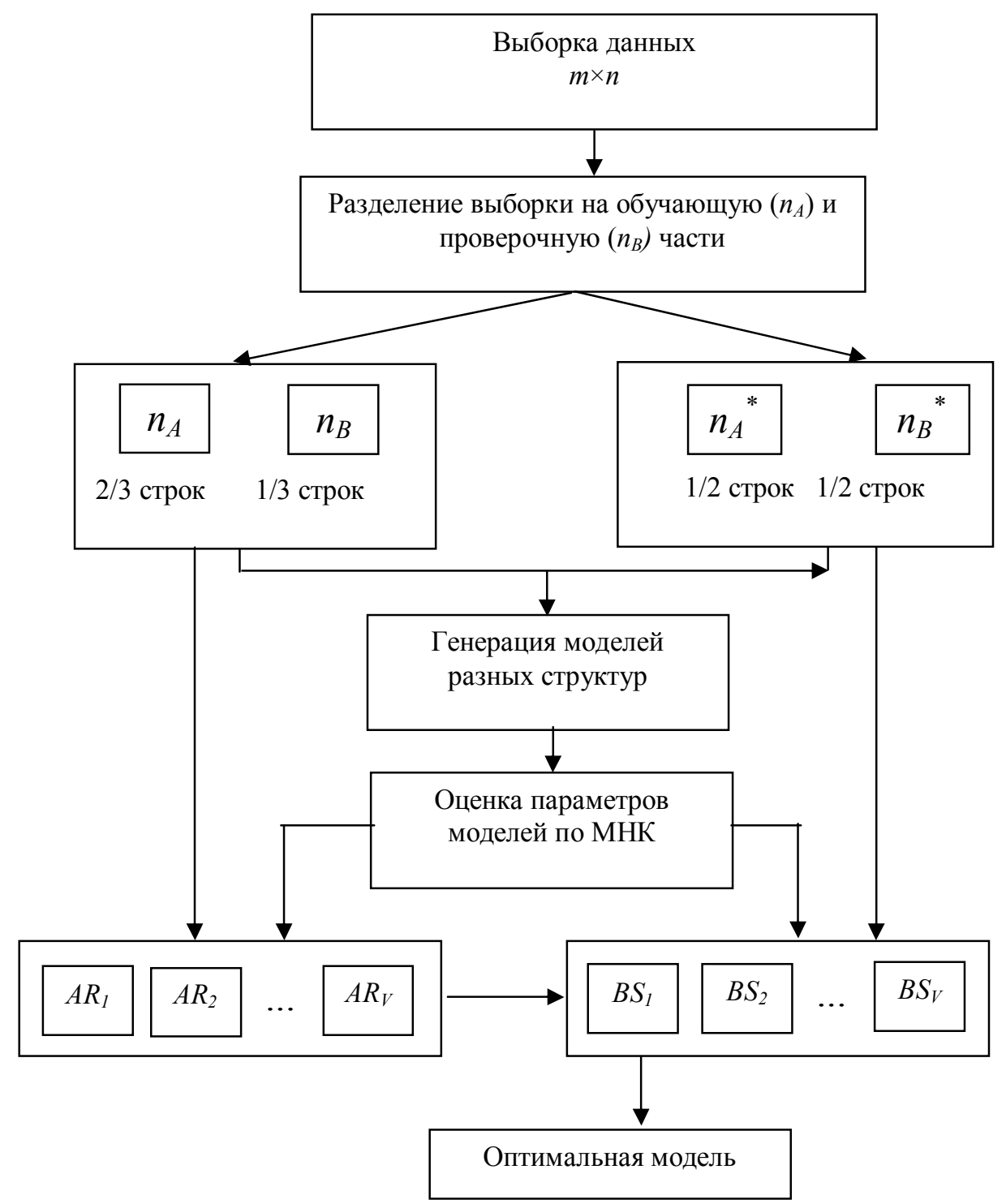

Рис. 2. Процедура доопределения по критерию несмещенности ошибок:

$n_{A}$ - длина обучающей части выборки данных; $n_{B}$ - длина проверочной части выборки данных; $A R_{1}, A R_{2}, \ldots, A R_{V}$ - значения критерия регулярности для лучших моделей, которые попали в интервал неопределенности; $B S_{1}, B S_{2}, \cdots, B S_{V} \quad$ значения критерия несмещенности для лучших по критерию регулярности моделей.

\section{ПРИМЕНЕНИЕ ТЕХНОЛОГИИ МОДЕЛИРОВАНИЯ И ПРОГНОЗИРОВАНИЯ ДЛЯ РЕШЕНИЯ ПРАКТИЧЕСКИХ ЗАДАЧ}

Проведено исследование эффективности описанной технологии на основе метода доопределения для выбора заданных моделей на тестовых примерах. Примеры показали, что полученная в результате работы данной 
технологии модель является наиболее точной не только на обучающей и проверочной частях выборки, но и на третьей, независимой, экзаменационной части [10].

Разработанная компьютерная технология апробирована при решении прикладных задач моделирования и прогнозирования: в задачах лечения диабета в условиях надомного мониторинга и задачах моделирования взаимодействия ионов струи газов с поверхностью материалов.

В задаче лечения диабета по данным надомного мониторинга проведено восстановление отсутствующих значений и получен краткосрочный прогноз уровня глюкозы в крови. Пример восстановления единичных пропусков исходных данных с различными шаблонами показал, что наиболее точными для восстановления единичных пропусков является крестообразный и квадратный шаблоны [9]. Выполнено сравнение результатов восстановления с МГУА и по методу экспоненциального сглаживания и показана эффективность первого метода.

При моделировании взаимодействия ионов струи газов с поверхностью материалов задача состояла в подборе материала покрытия поверхности космического аппарата, который содержит наименьший коэффициент распыления [11]. Совместно с учеными Московского авиационного института на основе экспериментальных данных, содержащих значения коэффициента распыления ряда материалов ионами ксенона при фиксированных параметрах газа, найдена зависимость коэффициента распыления от характеристик этих материалов. С помощью описанной технологии получена модель, которая имеет полиномиальную структуру, и хотя она выбрана не из физических соображений, но с помощью самоорганизации модели по внешнему критерию, оказалось, что она потенциально способна описать систему точнее, чем традиционные модели с физической параметризацией. Ошибка полученной модели около 20-40\%, в отличие от моделей с физической параметризацией, которые могут давать ошибку намного больше.

\section{Выводы}

Разработана и описана компьютерная технология, которая обеспечивает эффективное решение задач моделирования и прогнозирования по экспериментальным данным, что подтверждено тестовыми примерами. Эта технология обеспечивает повышение помехоустойчивости моделей за счет последовательного применения внешних критериев МГУА: критерия регулярности и несмещенности.

Применение разработанной технологии в решении реальных задач моделирования и прогнозирования показало ее эффективность.

1. Ивахненко А.Г. Помехоустойчивость моделирования / А.Г. Ивахненко, В.С. Степашко. - Киев : Наукова думка, $1985 .-215$ с.

2. Madala H.R. Inductive Learning Algorithms for Complex Systems Modeling / H.R. Madala, A.G. Ivakhnenko - Boca Raton: CRC Press Inc., 1994. — 384 p. 
3. Ивахненко А.Г. Метод группового учета аргументов - конкурент метода стохастической аппроксимации / А.Г. Ивахненко // Автоматика. - 1968. — № 3. C. $58-72$.

4. Степашко В.С. Комбинаторный алгоритм МГУА с оптимальной схемой перебора моделей / В.С. Степашко // Автоматика. — 1981. - № 3. - С.31-36.

5. Ивахненко А.Г. Исследование эффективности метода доопределения выбора модели в задачах моделированием с применением МГУА / А.Г.Ивахненко, Е.А. Савченко // Проблемы управления и информатики. - 2008. - № 2. - С. 65-76.

6. Савченко Е.А., Степашко В.С. Аналитическое и численное исследование селективных свойств критерия несмещенности ошибок в задачах индуктивного моделирования / Е.А. Савченко, В.С. Степашко // Проблемы управления и информатики. - № 2. 2012. - С. 39-50.

7. Ивахненко А.Г. Концепция последовательных алгоритмических приближений (спусков) к точному решению интерполяционных задач искусственного интеллекта / А.Г. Ивахненко, Г.А. Ивахненко, Е.А. Савченко // Кибернетика и вычислительная техника. - № 124. - 1999. - С. 40-60.

8. Савченко Е.А. Предварительная обработка выборки данных в задаче индуктивного моделирования / Е.А. Савченко // Управляющие системы и машины. - № 2. 2015. - С. 82-87.

9. Ивахненко А.Г. Применение алгоритмов МГУА для восстановления пропущенных данных и прогноза уровня глюкозы в крови при надомном мониторинге диабета / А.Г. Ивахненко, Е.А. Савченко, Г.А. Ивахненко, Т. Гергей // Проблемы управления и информатики. - 2002. - № 3. - С. 123-133.

10. Савченко Е. А. Анализ селективных свойств критериев МГУА при их последовательном применении / Е.А. Савченко, В.С. Степашко // Моделювання та керування станом еколого-економічних систем регіону. Збірник праць. - Київ: МННЦ ITC, 2008. - C. 199-210.

11. Ивахненко А.Г. Обнаружение закономерностей взаимодействия ионов с поверхностью по комбинаторному алгоритму МГУА / А.Г. Ивахненко, Е.А. Савченко, Г.А. Ивахненко [та ін.] // Проблемы управления и информатики. — 2003. — № 2. C. $80-89$.

UDC 681.513

\section{TECHNOLOGY FOR SOLVING THE PROBLEM OF MODELING AND FORECASTING BASED ON INDUCTIVE APPROACH}

\section{E.A. Savchenko}

International Research and Training Center for Information Technologies and Systems of National Academy of Sciences of Ukraine and Ministry of Education and Science of Ukraine

Introduction. The advantage of inductive algorithms is in their ability to automatically find dependencies hidden in a sample of experimental data. Combinatorial algorithms of GMDH (group method of data handling) are the main inductive modeling algorithms. These algorithms applied to real problems showed that it's not always possible to unambiguously determine a model by one criterion. Method of a model after-determination based on the Combinatorial GMDH algorithm is developed for such case. A technology based on the combinatorial GMDH algorithm and the after-determination method was developed for the modeling and forecasting.

The purpose of this article is to develop the technology for modeling and forecasting on the experimental data sample based on the combinatorial algorithm 
GMDH method and the after-determination method. This technology will help to find the optimal model in real applications.

Results. A technology for solving the problem of modeling and forecasting on the basis of the inductive approach was developed and described. This approach is based on the combinatorial algorithm GMDH method and completions. This technology, based on a sample of experimental data, automatically finds the object model or process using two external selection criteria: accuracy and bias. The developed computer technology was tested in solving applied problems of modeling and prediction: in problems of diabetes in a home-based monitoring and problems of modeling the interaction of ions with the surface of the jet gas materials.

Conclusion. A computer technology that provides an effective solution for the problems of modeling and prediction of the experimental data was developed and described. Numerical examples demonstrate its efficiency. This technology provides increased noise immunity models due to the consistent application of external criteria GMDH: the criterion of regularity and bias. This technology was used in real applications for modeling and forecasting and its effectiveness has been confirmed.

Keywords: inductive approach, combinatorial algorithm of group method of data handling, modeling, forecasting, technology.

1. Ivakhnenko A.G. Noise-immunity of modeling. Kiev: Naukova Dumka, 1985, 215 p. (in Russia).

2. Madala H.R. Inductive Learning Algorithms for Complex Systems Modeling. Boca Raton: CRC Press Inc., 1994, 384 p.

3. Ivakhnenko A.G. Group method of data handling as competitor to the method of stochastic approximation. Soviet Automatic Control, 1968, no. 3, pp. 64-78.

4. Stepashko V.S. Combinatorial GMDH algorithm with optimal scheme of models sorting. Automatics, 1981, no. 3, pp. 31-36 (In Russian).

5. Ivakhnenko A.G. Investigation of Efficiency of Additional Determination Method of the Model Selection in the Modeling Problems by Application of the GMDH Algorithm. Journal of Automation and Information sciences. Begell House: Inc. Publishers, 2008, vol. 40, no. 3 , pp. 47-58.

6. Savchenko E.A. Analytical and Numerical Study of the Selective Properties of the Errors Unbiasedness Criterion in the Problems of Inductive Modeling. Journal of Automation and Information sciences. Begell House: Inc. Publishers, 2012, vol. 44, no. 4, pp. 1-12.

7. Ivahnenko A.G. Conception of the successive algorithmic approaching (lowering) to the exact decision of interpolation tasks of artificial intelligence. Cybernetics and computing engineering, 1999, vol. 124, pp. 40-60 (In Russian).

8. Savchenko E.A. Preprocessing of data sample in inductive modeling problem. Control Systems and Computers, 2015, N2, pp. 82-87.

9. Ivakhnenko A.G. Application of Algorithms of the Method of Batch Assessment of Arguments for Recovering Missed Data and Prediction of the Glucose Level in Blood on at Home Diabetes Monitoring. Journal of Automation and Information sciences. Begell House: Inc. Publishers, 2002, vol. 34, no. 6, pp.123-133.

10. Savchenko E.A. Analysis of the selective properties of the GMDH criteria when applying them consistently. Modeling and control as ecological and economic systems of the region. Kyiv: IRTC ITS, 2008, no. 4, pp. 199-210 (In Russian). 


\title{
Интеллектуальное управление
}

\section{и системы}

УДК 519.8

\section{КРИТЕРИЙ МИНИМАКСНОГО СОЖАЛЕНИЯ В ЗАДАЧЕ ПРИНЯТИЯ РЕШЕНИЙ С НЕЧЕТКИМ МНОЖЕСТВОМ СОСТОЯНИЙ ОКРУЖАЮЩЕЙ СРЕДЫ}

\author{
С.О. Мащенко ${ }^{1}$, Ю.В. Шушарин ${ }^{2}$ \\ ${ }^{1}$ Киевский национальный университет им. Тараса Шевченко \\ ${ }^{2} \mathrm{Ku}$ \\ иевский национальный экономический университет им. Вадима Гетьмана
}

Предлагается метод принятия решений в условиях неопределенности с нечетким множеством состояний окружающей среды, который основан на принципе минимаксного сожаления. Определено понятие нечеткого множества гарантированных сожалений, построена его функция принадлежности. Рассмотрен подход к выбору лучшей по полезности альтернативы со степенью принадлежности нечеткому множеству гарантированных сожалений не менее заданного числа.

Ключевые слова: принятие решений, неопределенность, нечеткое множество, критерий Сэвиджа, максимизирующее решение.

Пропонується метод прийняття рішень в умовах невизначеності з нечіткою множиною станів навколишнього середовища, який заснований на принципі мінімаксного жалю. Визначено поняття нечіткої множини гарантованих жалів, побудована їі функція належності. Розглянуто підхід до вибору кращої за корисністю альтернативи за ступенем належності нечіткій множині гарантованих жалів не менше заданого числа.

Ключові слова: прийняття рішень, невизначеність, нечітка множина, критерій Севіджа, максимізуюче рішення.

\section{ВВЕДЕНИЕ}

Одной из актуальных проблем принятия решений является проблема выбора в условиях неопределенности состояний окружающей среды. Об этом свидетельствуют многочисленные ссылки на публикации в этом направлении, представленные в работах [1-3].

В общем случае задача принятия решений (ЗПР) в условиях неопределенности определяется на триаде множеств: $X-$ множество альтернатив; $Y$ - множество исходов; $S$ - множество состояний окружающей среды. Альтернативы - это то, что выбирает лицо, принимающее решение (ЛПР). Альтернативами могут служить объекты различной природы, планы, программы, действия и т.п. Исходы характеризуют результат выбора альтернатив. В ЗПР в условиях 
неопределенности каждой альтернативе может отвечать множество исходов, и поэтому связь между альтернативами и исходами может быть неоднозначной. Множество $S$ характеризует проявление неопределенности в принятии решений, причем конкретная интерпретация состояний зависит от постановки задачи (например, спрос на ту или другую продукцию, погода и т.п.).

Поскольку любой исход ЗПР в нормальной форме [1] однозначно определяется парой $(x, s) \in X \times S$, то ЛПР может задать функцию полезности исходов $u(x, s)$ на множестве $X \times S$. Цель ЛПР заключается в выборе альтернативы $x \in X$, максимизирующей функцию полезности исходов $u(x, s)$.

Таким образом, возникает задача выбора некоторой альтернативы, удовлетворяющей условиям

$$
v(x, s) \rightarrow \max _{x \in X}, s \in S
$$

Известно достаточно много разнообразных подходов к решению ЗПР в нормальной форме [1]. Основная идея решения задачи (1) заключается в построении так называемого критерия $E(x)$ (функции полезности альтернатив) [1], вид которого зависит от наличия некоторой дополнительной информации: о состояниях окружающей среды (чаще всего связанной с распределением вероятностей на множестве $S$ ), об особенностях ЛПР (склонность к риску и т. п.).

\section{ПОСТАНОВКА ЗАДАЧИ}

Нечеткий критерий минимаксного сожаления принятия решений в условиях неопределенности с нечетким множеством состояний окружающей среды, который мы будем рассматривать, основывается на известном критерии Сэвиджа [1] (принцип относительного пессимизма, или принцип минимаксного сожаления). Для каждого состояния окружающей среды $s \in S$ ЛПР определяет наибольшее значение функции полезности исходов, а именно, вычисляет $\max _{z \in X} v(z, s)$.

Разность $u(x, s)=\max _{z \in X} v(z, s)-v(x, s)$ выражает «сожаление» ЛПР о том, что для состояния окружающей среды $s \in S$ он использует $x$, а не альтернативу $x^{*}$, для которой $v\left(x^{*}, s\right)=\max _{z \in X} v(z, s)$. Затем ЛПР стремится выбрать такое решение $x \in X$, при котором максимально возможное (гарантированное) сожаление

$$
E_{S}(x)=\max _{s \in S} u(x, s)
$$


будет наименьшим. Таким образом, искомая альтернатива $x^{*}$ выбирается из условия

$$
E_{S}\left(x^{*}\right)=\min _{x \in X} E_{S}(x)
$$

ЦЕль. В некоторых случаях может быть так, что ЛПР не может четко задать множество состояний окружающей среды, которые будут влиять на исходы принятия решений задачи (3). Цель данной работы состоит в реализации принципа минимаксного сожаления в этих условиях.

\section{НЕЧЕТКОЕ МНОЖЕСТВО ГАРАНТИРОВАННЫХ СОЖАЛЕНИЙ}

Пусть $\widetilde{S}$ - некоторое нечеткое множество универсального множества состояний окружающей среды $S$ с функцией принадлежности $\mu(s)$, $\mu: S \rightarrow[0,1]$. В этом случае для каждой фиксированной альтернативы $x \in X$ критерий (2) принимает вид задачи нечеткого математического программирования [2]:

$$
\widetilde{E}_{S}(x)=\max _{s \in \widetilde{S}} u(x, s)
$$

Задачи нечеткого математического программирования достаточно изучены. Согласно [3] считается, что для каждой фиксированной альтернативы $x \in X$ ЛПР хочет максимизировать как ее функцию полезности $u(x, s)$, так и функцию $\mu(s), s \in S$, принадлежности нечеткого множества состояний окружающей среды $\widetilde{S}$. Решением задачи (4) для фиксированной альтернативы $x \in X$ называется нечеткое множество состояний окружающей среды $S^{*}(x)$, носителем которого будет множество оптимальных по Парето решений (обозначим его $\left.S^{P O}(x)\right)$ двухкритериальной задачи оптимизации:

$$
u(x, s) \rightarrow \max , \quad \mu(s) \rightarrow \max , s \in S .
$$

Напомним, что для задачи (5) множество $S^{P O}(x)$ можно представить в виде:

$$
S^{P O}(x)=\left\{s^{*} \in S \mid\left(\mu(s)<\mu\left(s^{*}\right)\right) \vee\left(u(x, s)<u\left(x, s^{*}\right)\right), \forall s \in S\right\} .
$$

Функцией принадлежности $\pi_{x}(s)$ нечеткого множества $S^{*}(x)$ будет сужение функции принадлежности $\mu(s), s \in S$, с универсального множества $S$ на множество $S^{P O}(x) \subseteq S$. Другими словами, эта функция принадлежности будет иметь вид: 


$$
\pi_{x}(s)= \begin{cases}\mu(s), & s \in S^{P O}(x), \\ 0, & s \notin S^{P O}(x) .\end{cases}
$$

Множеству решений задачи (5), которым является нечеткое множество $S^{*}(x)$ с функцией принадлежности $\pi_{x}(s), s \in S$, согласно [3] отвечает нечеткое множество $\widetilde{E}_{S}(x) \subseteq E(x) \subseteq R^{1}$ оптимальных значений целевой функции этой задачи с функцией принадлежности

$$
\varphi_{x}(y)=\max _{s \in S, u(x, s)=y} \pi_{x}(s), \quad y \in E(x),
$$

где $E(x)=\{u=u(x, s) \mid s \in S\} \subseteq R^{1}$ - универсальное множество возможных полезностей альтернативы $x \in X$ при всех возможных состояниях окружающей среды.

На основе представленных выше рассуждений определим понятие нечеткого множества гарантированных сожалений.

Нечетким множеством гарантированных сожалений для альтернативы $x \in X$ ЗПР в условиях неопределенности с нечетким множеством состояний окружающей среды $\widetilde{S}$ с функцией принадлежности $\mu(s), s \in S$, будем называть нечеткое множество $\widetilde{E}_{S}(x) \subseteq R^{1}$ с функцией принадлежности

$$
\varphi_{x}(y)=\max _{s \in S, u(x, s)=y} \pi(x, s) .
$$

Значение $y \in E(x)$ будем называть гарантированным сожалением для альтернативы $x \in X$, а величину $\varphi_{x}(y)$ - ее степенью принадлежности множеству $\widetilde{E}_{S}(x)$.

Определим $E \supseteq \bigcup_{x \in X} E(x), \quad E \subseteq R^{1}-$ универсальное множество возможных сожалений для альтернатив универсального множества $X$ при всех возможных состояниях окружающей среды. Отметим, что для всего множества $X$ функция $\varphi_{x}(y)$ определяет функцию принадлежности $\varphi(x, y)$ некоторого нечеткого отображения $\widetilde{\Phi}$ из множества альтернатив $X$ в множество сожалений $E \subseteq R^{1}$ для них:

$$
\varphi(x, y)=\left\{\begin{array}{cl}
\max _{s \in S, u(x, s)=y} \pi(x, s), & y \in E(x), \\
0, & y \in E \backslash E(x) .
\end{array}\right.
$$

Таким образом, выходит, что в случае нечеткого множества состояний окружающей среды альтернативы $x \in X$ нужно сравнивать между собой по соответствующим им нечетким множествам $\widetilde{E}_{V}(x)$ значений сожалений. 
Поэтому решением задачи будет нечеткое множество, которое обеспечивает наилучшее гарантированное сожаление. Для реализации этой идеи используем технику, которая развита в [3].

\section{НАИЛУЧШЕЕ ГАРАНТИРОВАННОЕ СОЖАЛЕНИЕ}

Обозначим $\mu_{R}: E \times E \rightarrow[0,1]-$ функцию принадлежности четкого отношения $R$, которое является отношением «не больше», заданного на $E \subseteq R^{1}$. Очевидно, что

$$
\mu_{R}(z, y)= \begin{cases}1, & z \leq y \\ 0, & z>y\end{cases}
$$

Для решения поставленной задачи построим на множестве альтернатив $X$ нечеткое отношение предпочтения, индуцируемое исходным отношением предпочтения $R$ и нечеткой целью, которая задана нечетким отображением $\widetilde{\Phi}$. После этого выделим в $X$ нечеткое подмножество недоминируемых альтернатив, которое будет множеством решений задачи (2).

Произвольной альтернативе $x^{*}$ заданное нечеткое отображение $\widetilde{\Phi}$ ставит в соответствие нечеткое множество сожалений для этой альтернативы в форме нечеткого подмножества множества сожалений $E \subseteq R^{1}$ с функцией принадлежности $\varphi\left(x^{*}, y\right)$.

Пусть $\tilde{\eta}$ - нечеткое отношение предпочтения, индуцируемое отношением предпочтения $R$ на классе $\Psi$ всех нечетких подмножеств множества $E$. Пользуясь этим отношением, можно сравнивать между собой нечеткие сожаления для альтернатив, а следовательно, и сами альтернативы. Другими словами, степенью предпочтения альтернативы $x_{1} \in X$ альтернативе $x_{2} \in X$ будем считать степень предпочтения $\eta\left(x_{1}, x_{2}\right)$ нечеткого множества сожалений $\varphi\left(x_{1}, y\right)$ нечеткому множеству сожалений $\varphi\left(x_{2}, y\right)$.

Таким образом, используя определение [3] обобщенного нечеткого отношения предпочтения, получим нечеткое отношение предпочтения на множестве альтернатив $X$ следующего вида:

$$
\eta\left(x_{1}, x_{2}\right)=\max _{z, y \in E} \min \left\{\varphi\left(x_{1}, z\right), \varphi\left(x_{2}, y\right), \mu_{R}(z, y)\right\} .
$$

Нетрудно убедиться в том, что когда функция принадлежности $\varphi$ удовлетворяет условию

$$
\max _{y \in E} \varphi(x, y)=1, \quad \forall x \in X,
$$


т. е. когда множество сожалений для произвольной альтернативы образует нормальное нечеткое множество, то нечеткое отношение предпочтения $\eta$ будет рефлексивним, т. е. $\forall x \in X \eta(x, x)=1$.

После того, как и на множестве альтернатив определено нечеткое отношение предпочтения, исходная задача сводится к ЗПР с целью, заданной нечетким отношением предпочтения [3].

Выделим теперь во множестве $X$ наилучшее гарантированное сожаление как нечеткое множество недоминируемых альтернатив. Согласно [3] оно будет иметь вид:

$$
\begin{gathered}
\widetilde{\eta}^{P O}(x)=1-\max _{x^{\prime} \in X}\left\{\eta\left(x^{\prime}, x\right)-\eta\left(x, x^{\prime}\right)\right\}= \\
=1-\max _{x^{\prime} \in X}\left\{\max _{z, y \in E} \min \left\{\varphi\left(x^{\prime}, z\right), \varphi(x, y), \mu_{R}(z, y)\right\}-\right. \\
\left.-\max _{z, y \in E} \min \left\{\varphi(x, z), \varphi\left(x^{\prime}, y\right), \mu_{R}(y, z)\right\}-\max _{z, y \in E} \min \left\{\varphi(x, z), \varphi\left(x^{\prime}, y\right), \mu_{R}(y, z)\right\}\right\} .
\end{gathered}
$$

Тогда согласно (9) окончательно получаем:

$$
\widetilde{\eta}^{P O}(x)=1-\max _{x^{\prime} \in X}\left\{\max _{\substack{z, y \in E \\ z \leq y}} \min \left\{\varphi\left(x^{\prime}, z\right), \varphi(x, y)\right\}-\max _{\substack{z, y \in E \\ z \leq y}} \min \left\{\varphi(x, z), \varphi\left(x^{\prime}, y\right)\right\}\right\} .
$$

Необходимо отметить, что если функция $\varphi(x, y)$ такова, что для некоторой альтернативы $x^{*}$ имеет место неравенство $\sup \varphi\left(x^{*}, y\right)=\alpha<1$, то $y \in E$

значение $\widetilde{\eta}^{P O}\left(x^{*}\right)$ может не отвечать фактической степени недоминируемости этой альтернативы.

Для того, чтобы исключить такие аномальные случаи, величину $\widetilde{\eta}^{P O}(x)$ необходимо скорректировать. Для этого значения функции $\widetilde{\eta}^{P O}(x)$ нужно сравнивать с соответствующими значениями $\max _{y \in E} \varphi(x, y)$.

Опираясь на эти рассуждения, решением исходной задачи будем считать нечеткое множество не с функцией принадлежности $\widetilde{\eta}^{P O}$, а со скорректированной функцией

$$
\eta^{P O}(x)=\min \left\{\widetilde{\eta}^{P O}(x), \max _{y \in E} \varphi(x, y)\right\} .
$$

Нетрудно показать, что для произвольного $x$ имеет место равенство $\max \varphi(x, y)=\eta(x, x)$. Таким образом, приходим к следующему понятию. $y \in E$ 
Нечеткое множество $X^{*}$ с функцией принадлежности

$$
\eta^{P O}(x)=\min \left\{\widetilde{\eta}^{P O}(x), \eta(x, x)\right\}
$$

будем называть решением ЗПР с нечетким множеством состояний окружающей среды (2) по нечеткому критерию минимаксного сожаления.

Если вдруг ЛПР по каким-либо причинам потребуется максимизирующее решение $x^{*}$, то оно может быть найдено как $\max _{x \in X} \eta^{P O}(x)$.

В некоторых случаях ЛПР может интересовать лучшая по сожалению альтернатива со степенью принадлежности нечеткому множеству $X^{*}$ решений не менее заданного числа $\alpha \in(0,1]$. В [3] показано, что для ее нахождения достаточно решить задачу математического программирования:

$$
\begin{aligned}
& y \rightarrow \min , \\
& \varphi(x, y) \geq \alpha, \\
& x \in X, \quad y \in E \subseteq R^{1} .
\end{aligned}
$$

Решение задачи (10) можно существенно упростить, если использовать следующую теорему.

Теорема. Предположим, что функиия принадлежности нечеткого множества состояний окружающей среды $\mu(s)>0 \quad \forall s \in S$. Альтернатива $x^{*} \in X$ будет лучшей по сожалению со степенью принадлежности множеству решений $X^{*}$ не менее заданного числа $\alpha \in(0,1]$ по нечеткому критерию минимаксного сожаления тогда и только тогда, когда будет удовлетворять системе двух задач математического программирования:

$$
\begin{gathered}
u\left(x^{*}, s^{*}\right)=\min _{x \in X} \max _{s \in S, \mu(s) \geq \alpha} u(x, s), \\
\mu\left(s^{*}\right)=\max \left\{\mu(s) \mid u\left(x^{*}, s\right) \geq u\left(x^{*}, s^{*}\right)\right\}, \quad s \in S .
\end{gathered}
$$

Доказательство. Пусть $x^{*} \in X \quad$ и $s^{*} \in S$ удовлетворяют (11) (12). Покажем, что $x^{*}$ и $y^{*}=u\left(x^{*}, s^{*}\right)$ удовлетворяют (10). Действительно, из (11), (12) следует, что

$$
\begin{gathered}
u\left(x^{*}, s\right) \leq u\left(x^{*}, s^{*}\right) \leq u\left(x, s^{*}\right), \quad \forall x \in X, \\
\forall s \in\{s \in S \mid \mu(s) \geq \alpha\} \\
\mu\left(s^{*}\right) \geq \alpha
\end{gathered}
$$




$$
\mu\left(s^{*}\right) \geq \mu(s), \quad \forall s \in\left\{s \in S \mid u\left(x^{*}, s\right) \geq u\left(x^{*}, s^{*}\right)\right\} .
$$

Покажем, что $s^{*} \in S^{P O}\left(x^{*}\right)$. Предположим противное: $s^{*} \notin S^{P O}\left(x^{*}\right)$. Тогда согласно (6) $\exists s \in S$, для которого выполняются неравенства:

$$
\mu(s) \geq \mu\left(s^{*}\right), \quad u\left(x^{*}, s\right)>u\left(x^{*}, s^{*}\right),
$$

или

$$
\mu(s)>\mu\left(s^{*}\right), \quad u\left(x^{*}, s\right) \geq u\left(x^{*}, s^{*}\right) .
$$

Если имеют место неравенства (16), то получаем противоречие с (13), (14). Если же справедливы неравенства (17), то получим противоречие с (15). Таким образом, $s^{*} \in S^{P O}\left(x^{*}\right)$.

Предположим противное, что $x^{*}$ и $y^{*}=u\left(x^{*}, s^{*}\right)$ не удовлетворяют (10). Возможны следующие случаи.

1. Предположим, что $\varphi\left(x^{*}, y^{*}\right)<\alpha$. Тогда $\underset{s \in S, u\left(x^{*}, s\right)=y^{*}}{\max } \pi\left(x^{*}, s\right)<\alpha$. Отсюда $\pi\left(x^{*}, s\right)<\alpha$ для таких $s \in S$, что $u\left(x^{*}, s\right)=y^{*}$. В том числе и для $s^{*}$. Поэтому $\pi\left(x^{*}, s^{*}\right)<\alpha . \quad$ Поскольку $s^{*} \in S^{P O}, \quad$ то $\quad \pi\left(x^{*}, s^{*}\right)=\mu\left(s^{*}\right)$. Отсюда $\mu\left(s^{*}\right)<\alpha$, что противоречит (14).

2. Предположим, что $\exists \bar{y}<y^{*}$ и $\exists x \in X$ такие, что

$$
\varphi(x, \bar{y}) \geq \alpha, \quad \bar{y} \in E(x)=\{y=u(x, s) \mid s \in S\} .
$$

Это означает, что $\exists \bar{s} \in S \quad$ и $\exists x \in X$, для которых выполняются неравенства:

$$
\begin{gathered}
\varphi(x, u(x, \bar{s})) \geq \alpha, \\
u(x, \bar{s})<u\left(x^{*}, s^{*}\right) .
\end{gathered}
$$

Из (18) следует, что $\varphi(x, u(x, \bar{s}))=\max _{s \in S, u(x, s)=u(x, \bar{s})} \pi(x, s) \geq \alpha$. Отсюда $\exists \tilde{s} \in S$, для которого $\pi(x, \tilde{s}) \geq \alpha \quad$ и $u(x, \widetilde{s})=u(x, \bar{s})$. Тогда из (19) следует неравенство $u(x, \tilde{s})<u\left(x^{*}, s^{*}\right)$. Поэтому $\widetilde{s} \notin S^{P O}\left(x^{*}\right)$. Тогда $\pi(x, \tilde{s})=0$ и мы получаем противоречие $0 \geq \alpha>0$.

Условие достаточности теоремы доказано.

Покажем необходимость. Пусть $y^{*}$ удовлетворяет (10). Отсюда следуют неравенства

$$
y^{*} \leq y \quad \forall y \in Y=\left\{y \in R^{1} \mid \varphi(x, y) \geq \alpha, y \in E(x), x \in X\right\} .
$$


Поскольку на основании (8)

$$
\varphi(x, y)=\max _{s \in S, u(x, s)=y} \pi(x, s)
$$

то эти неравенства можно переписать в виде:

$$
y^{*} \leq y \quad \forall y \in Y=\left\{y \in R^{1} \mid \max _{s \in S, u(x, s)=y} \pi(x, s) \geq \alpha, y \in E(x), x \in X\right\} .
$$

Обозначим через $x^{*} \in X$ такую альтернативу, что $y^{*}=u\left(x^{*}, s^{*}\right)$, где состояние окружающей среды $s^{*}=\arg \underset{s \in S, u\left(x^{*}, s\right)=y^{*}}{\max } \pi\left(x^{*}, s\right)$. Отметим, что поскольку по предположению задача (10) имеет решение, то и (20) имеет решение. Поэтому $x^{*}$ и $s$ с существуют. Тогда

$$
\pi\left(x^{*}, s^{*}\right) \geq \alpha \text {. }
$$

На этом основании (20) можно переписать в виде

$$
\begin{gathered}
u\left(x^{*}, s^{*}\right) \leq u(x, s) \quad \forall x \in X \\
\forall u(x, s) \in\left\{u(x, s) \in E(x) \mid \max _{v \in S, u(x, v)=u(x, s)} \pi(x, v) \geq \alpha\right\} .
\end{gathered}
$$

Эти неравенства, в свою очередь, можно записать таким образом:

$$
\begin{gathered}
u\left(x^{*}, s^{*}\right) \leq u(x, s) \\
\forall x \in X \quad \forall s \in S(x)=\left\{s \in S \mid \max _{v \in S, u(x, v)=u(x, s)} \pi(x, v) \geq \alpha\right\} .
\end{gathered}
$$

Из (22), в частности, следует, что для $s=s^{*}$ справедливы неравенства

$$
u\left(x^{*}, s^{*}\right) \leq u\left(x, s^{*}\right) \quad \forall x \in X .
$$

Также из (22), в частности, следует, что для фиксированного $x=x^{*}$

$$
u\left(x^{*}, s^{*}\right) \leq u\left(x^{*}, s\right) \quad \forall s \in S\left(x^{*}\right)=\left\{s \in S \mid \max _{v \in S, u\left(x^{*}, v\right)=u\left(x^{*}, s\right)} \pi\left(x^{*}, v\right) \geq \alpha\right\} .
$$

Поскольку согласно постановке задачи (10) $\alpha>0$, то из (24), очевидно, выплывает, что $\pi\left(x^{*}, \bar{s}\right)>0 \quad \forall \bar{s} \in S\left(x^{*}\right)$. Поэтому в соответствии с предположением теоремы о том, что $\mu(s)>0 \forall s \in S$, учитывая (7), получаем

$$
\mu(\bar{s})=\pi\left(x^{*}, \bar{s}\right) \geq \alpha,
$$




$$
\bar{s} \in S^{P O}\left(x^{*}\right) \quad \forall \bar{s} \in S\left(x^{*}\right) .
$$

Таким образом, из (25) и (24) следует неравенство

$$
u\left(x^{*}, s^{*}\right) \leq u\left(x^{*}, s\right) \quad \forall s \in S\left(x^{*}\right)=\{s \in S \mid \mu(s) \geq \alpha\} .
$$

Отметим также, что следствием (21) и (24) является включение

$$
s^{*} \in S\left(x^{*}\right) \text {. }
$$

Поэтому из (25), (26), (28) получаем

$$
\begin{gathered}
\mu\left(s^{*}\right) \geq \alpha, \\
s^{*} \in S^{P O}\left(x^{*}\right) .
\end{gathered}
$$

Покажем, что пара $x^{*}, s^{*}$ удовлетворяет (13) - (15). Предположим противное, что условия (13) - (15) не выполняются. Тогда возможны следующие случаи.

1. Предположим, что $\exists x \in X \quad u\left(x^{*}, s^{*}\right)>u\left(x, s^{*}\right)$. Тогда получаем противоречие с (23).

2. Предположим, что $\exists \bar{s} \in S$, для которого $\mu(\bar{s}) \geq \alpha$ и $u\left(x^{*}, s^{*}\right)>u\left(x^{*}, \bar{s}\right)$. В этом случае получаем противоречие с (27).

3. Предположим $\mu\left(s^{*}\right)<\alpha$. Тогда получаем противоречие с (29).

4. Предположим, что $\exists \bar{s} \in S$, для которого выполняются неравенства $\mu\left(s^{*}\right)<\mu(\bar{s})$ и $\left.u\left(x^{*}, \bar{s}\right) \geq u\left(x^{*}, s^{*}\right)\right\}$. В этом случае согласно (6) получаем противоречие с (30).

Таким образом, пара $x^{*}, s^{*}$ удовлетворяет (13) - (15) и задачи (10) и (11), (12) имеют одни и те же решения.

Теорема доказана.

\section{Выводы}

Рассмотренный выше метод решения задачи принятия решений в условиях неопределенности с нечетким множеством состояний окружающей среды можно обобщить на случай нечеткого множества альтернатив и нечетких оценок полезности исходов. Для этого достаточно использовать известные методы дефаззификации [3].

1. Трухаев Р.И. Модели принятия решений в условиях неопределенности / Р.И. Трухаев. - М.: Наука, 1981. — 258 с.

2. Мащенко С.О. Обобщение критерия Гермейера в задаче принятия решений в условиях неопределенности с нечетким множеством состояний природы / С.О. Мащенко // Проблемы управления и информатики. — 2012. — № 5 . - С. 102-110.

(C) С.О. Мащенко, Ю.В. Шушарин, 2015 
3. Орловский С.А. Проблемы принятия решений при нечеткой исходной информации / С.А. Орловский. — М.: Наука, 1981. — 208 с.

\section{UDC 519.8 ENVIRONMENT STATES

\author{
S.O. Mashchenko ${ }^{1}$, Yu.V. Shusharin ${ }^{2}$ \\ ${ }^{1}$ Taras Shevchenko National University of Kyiv \\ ${ }^{2}$ Kyiv National Economic University
}

MINIMAX REGRET CRITERION FOR DECISION MAKING PROBLEM WITH THE FUZZY SET OF

Introduction. In this work, the problem of alternatives rational choice in the conditions of uncertainties with the fuzzy set of states of the environment is considered. A need for the solving of such problems arises, when a decision making person cannot expressly indicate, what states of environment will affect the consequences of the alternatives choice in a problem which appeared at the moment of the decision making. In this case, it can only set the belonging function of a fuzzy set of relevant environment states.

The purpose of the article is the application of the known minimax regret principle for construction of a fuzzy criterion of minimax regret in the conditions of uncertainties with the fuzzy set of environment states.

Methods. The methods of the fuzzy set theory, fuzzy mathematical programming, multicriterion optimization are used in this work.

Results. It is suggested to estimate every alternative of the decision making problem by a fuzzy set of the guaranteed regrets, which can be obtained as a set of maximal values function of results utility on the fuzzy set of environment states. The solving of the problem with a fuzzy set of environment states by the fuzzy criterion of minimax regret is determined as a fuzzy set of non-dominated alternatives according to a specially built fuzzy relation of preference. The method of choice of the best-by-regret alternative with the degree of membership to the fuzzy set of decisions not less than the set number is offered. For this purpose, it is suggested to solve the system of two problems of mathematical programming of the special kind.

Conclusion. The offered method of problem solving in the conditions of uncertainties with the fuzzy set of nature states can be easily generalized in the case of a fuzzy set of alternatives and fuzzy estimations of results utility. For this purpose, it is enough to use the known technique of defuzzification.

Keywords: decision making, uncertainties, fuzzy set, the L. Savage criterion, maximizing decision.

1. Truhaev R.I. Models of decision makig in the conditions of uncertainties. Moskow: Science, 1981, 258 p. (in Russian).

2. Mashchenko S.O. Generalization Germeyer's criterion in the decision making problem in conditions of uncertainty with the fuzzy set of nature states. Journal of Automation and Information Sciences, 2012, no. 5, pp. 102-110 (in Russian).

3. Orlovsky S.A. Problems of decision makig at fuzzy initial information. Moskow: Science, 1981, 208 p. (in Russian).

Получено 17.02.2015 


\title{
ИНВАРИАНТНАЯ СЕТЕЦЕНТРИЧЕСКАЯ СИСТЕМА УПРАВЛЕНИЯ КОНФЛИКТНЫМИ СИТУАЦИЯМИ ВОЗДУШНЫХ КОРАБЛЕЙ НА ЭТАПЕ ЗАХОДА НА ПОСАДКУ
}

\author{
В.В. Павлов, А.Е. Волков, Д.А. Волошенюк
}

Международный научно-учебный центр информационных технологий и систем НАН Украины и МОН Украины

Рассмотрен вопрос необходимости создания системы управления конфликтными ситуациями воздушных кораблей на этапе захода на посадку. Предлагается идея применения сетецентрических технологий для создания новой структуры системы управления воздушным движением в области гражданской авиации с целью обеспечения высокого гарантированного уровня безопасности полетов. Предложен подход к решению задачи, основанный на исследованиях в области дифференциальных игр и теории инвариантности.

Ключевые слова: сетецентрическая система, безопасность полетов, инвариантность, конфликтные ситуации, дифференциальные игры, свободный полет.

Розглянуто питання необхідності створення системи керування конфліктними ситуаціями повітряних кораблів на етапі заходу на посадку. Пропонується ідея застосування мережецентричних технологій для створення нової структури системи керування повітряним рухом в галузі цивільної авіації з метою забезпечення високого гарантованого рівня безпеки польотів. Запропоновано підхід до вирішення завдання, заснований на дослідженнях в області диференціальних ігор та теорії інваріантності.

Ключові слюва: мережецентрична система, безпека польотів, інваріантність, конфліктні ситуації, диференціальні ігри, вільний політ.

\section{ВВЕДЕНИЕ}

В соответствии со сформулированной стратегией развития организации воздушного движения Европейская организация по безопасности аэронавигации среди приоритетных направлений отвела важное место разработке новых принципов управления воздушным движением (УВД) и организации воздушного пространства, которые призваны обеспечить высокую пропускную способность сети маршрутов и возможность выполнять полеты по наиболее эффективным траекториям при гарантированном уровне безопасности, которого требуют современные авиационные стандарты.

Современные требования к УВД заключаются в следующем: увеличение пропускной способности системы УВД; гибкое использование воздушного пространства; возможность выполнять полеты по оптимальным траекториям; динамическая коррекция планов полетов; уменьшение временных задержек прибытия рейсов; минимизация отклонения самолетов от запланированных траекторий; обеспечение высокого гарантированного уровня безопасности полетов; упрощение и унификация процедур взаимодействия «землясамолет-земля» и т.д.

Для реализации этих требований была разработана концепции "FreeFlight" [1]. 
Основным заданием концепции "FreeFlight" является повышение эффективности воздушного движения в целом и оптимизация выполнения полета отдельного воздушного корабля (ВК) путем предоставления экипажу полной свободы в выборе траектории движения с соблюдением гарантированного уровня безопасности. Главной идеей концепции свободного полета «FreeFlight» является предоставление экипажу возможности выбирать траекторию полета по маршруту, скорости и профилю полета по приборам в большей степени, чем это позволяют правила визуального полета. Но такая автономность должна эффективно сосуществовать с надежностью безопасного расхождения ВК средствами бортовой аппаратуры.

Постепенный переход к полетам по произвольным маршрутам [2], который предусмотрено осуществить в течение ближайших 10-15 лет, несет в себе дополнительные трудности, такие, как рост количества и сложности потенциально-конфликтных ситуаций, возникающих между ВК. Все это влечет за собой снижение уровня безопасности полетов и, соответственно, именно этим обусловлена актуальность данной темы.

Соответственно, ставится задача создания математического и программного аппарата, позволяющего эффективно решать сложные конфликтные ситуации с произвольным количеством задействованных ВК, что является чрезвычайно актуальным для режима FreeFlight. Необходимым условием для обеспечения этого процесса является четкая координация действий между всеми ВК, согласно установленной последовательности решения конфликта.

Целью данной работы является разработка способа и системы решения конфликтных ситуаций ВК на маршрутах полета, этапах взлета и посадки, с условием обеспечения гарантированного уровня безопасности полетов, и применением сетецентрических технологий, основываясь на теории инвариантности.

\section{ПОСТАНОВКА ЗАДАЧИ}

Необходимость проводимых в работе исследований подтверждается тем, что современное состояние мировой авиатранспортной системы характеризуется стабильным увеличением объемов авиаперевозок, которое уже приводит к достижению системой своих предельных возможностей. Дальнейшее увеличение интенсивности воздушного движения неизбежно приводит к снижению безопасности полетов, увеличению задержек ВК как на земле, так и в воздухе, увеличению расхода топлива и снижению эффективности выполнения полетов, неприемлемому увеличению экологических издержек. Повышение интенсивности движения в европейской сети воздушных маршрутов непосредственно влияет на увеличение количества потенциально-конфликтных ситуаций, о чем свидетельствуют статистические данные. Как следствие, наземные диспетчерские центры испытывают значительные перегрузки при регулировании потоков воздушного движения. 
Столкновение ВК в воздухе друг с другом вызывает большое число катастроф, поэтому очень важна количественная оценка безопасности полета величиной допустимого риска столкновения воздушных судов. Однако, даже с помощью высокоэффективных и высоконадежных систем УВД невозможно обеспечить необходимую безопасность полетов. Дело в том, что до сих пор часть земной поверхности не охвачена зоной действия систем УВД, а кроме того, существующие системы УВД не позволяют надежно контролировать полеты на малых высотах и в труднодоступных для наблюдения регионах земного шара.

Одной из самых важных причин опасных сближений ВК является то, что диспетчер принимает решения по прогнозированию и определению конфликтов на основании неполной информации в условиях дефицита времени.

Ожидаемым результатом данной работы является создание новой универсальной системы управления конфликтными ситуациями воздушных кораблей, построенной на сетецентрических технологиях и на принципах теории инвариантности, которая позволит удовлетворить современным требованиям организации воздушного движения (ОВД) по обеспечению гарантированного уровня безопасности полетов.

Попытки провести классификацию современных методов и алгоритмов решения конфликтных ситуаций между ВК можно найти в работе [3]. Обобщенный вид этой классификации имеет весьма обширную и распределенную структуру и позволяет сделать несколько важных выводов:

- значительная часть методов и алгоритмов рассчитана на конфликтные ситуации с участием только двух ВК, а для более сложных случаев использует попарный перебор, при этом не учитывается возможное влияние решения одного конфликта на другие конфликты, которые расположены сравнительно недалеко по времени и расстоянию;

- наибольшая эффективность решения конфликтов достигается при использовании распределенных наземно-бортовых систем, которые пока полноценно не реализованы.

\section{АНАЛИЗ СОВРЕМЕННОГО СОСТОЯНИЯ ПРОБЛЕМЫ}

Глобальный аэронавигационный план Международной организации гражданской организации (ИКАО) на 2013-2028 года предусматривает повышение уровня безопасности и обслуживания в аэронавигации за счет создания новых систем предотвращения опасных сближений ВК, основанных на управлении цифровой аэронавигационной информацией (ЦАИ) [4]. Управление ЦАИ позволит сократить время, необходимое для распространения информации о статусе воздушного пространства, обеспечить более эффективное использование воздушного пространства, повысить качество передачи данных, упростить процедуры проверки данных, организовать поток воздушного движения с минимальными задержками, осуществлять мониторинг за эксплуатационной средой на этапе полета в целях обеспечения своевременной выдачи предупреждений об увеличении 
риска для безопасности полетов. Все это является технологической основой при создании новых систем предотвращения столкновений ВК.

Необходимо помнить, что одним из самых обширных классов систем управления является класс дифференциально-игровых систем или динамических игр [5].

В настоящее время достаточно хорошо развита теория динамических игр. Существующие подходы - прямые методы Л.С. Понтрягина, правило экстремального прицеливания Н.Н. Красовского, метод групповых операторов Б.Н. Пшеничного, техника, связанная с основными уравнениями теории дифференциальных игр Р. Айзекса, метод решающих функций, позиционные дифференциальные игры Н.Н. Красовского - А.И. Субботина и ряд других эффективных процедур, позволяют исследовать широкие классы конфликтно-управляемых процессов на предмет игрового сближения траекторий. Весьма разнообразны и убедительны методы уклонения от встречи: метод маневра обхода Л.С. Понтрягина - Е.Ф. Мищенко, методы постоянных и переменных направлений, метод инвариантных подпространств, рекурсивный метод и др.

Однако, большинство методов, в том числе и самые распространенные методы, предложенные Н.Н. Красовским в работе [6], где рассматривается игровая задача уклонения жестко скоординированных убегающих в шаре от группы инерционных преследователей и приводится подробный математический анализ этой проблемы, описывают только линейные и стационарные системы, в то время как полет самолета и конфликт ВК являются динамическим нелинейным процессом.

Большое внимание рассмотрению конфликтно-управляемых процессов уделено в работах А.А. Чикрия [7, 8]. Приведем предлагаемый им пример конфликтно-управляемого процесса в евклидовом пространстве. Для этого рассмотрим конфликтно-управляемый процесс в конечномерном евклидовом пространстве $R^{n}, n \geq 2$ :

$$
z(t)=g(t)+\int_{0}^{t} \Omega(t, \tau)(u(\tau), v(\tau)) d \tau .
$$

Вектор-функция $g(t), g: R_{+} \rightarrow R^{n}, R_{+}=\{t: t \geq 0\}$, измерима по Лебегу и почти везде ограниченна, матричная функция $\Omega(t, \tau)$ определена в треугольнике $\Delta=\{(t, \tau): t \geq \tau \geq 0\}$, измерима по $t$ и суммируема по $\tau$ для каждого $t \in R_{+}$. Блок управления задается функцией $\varphi(u, v), \varphi: U \times V \rightarrow R^{n}$, непрерывной по совокупной переменных на прямом произведении непустых компактов $U$ и $V, U \in K\left(R^{m}\right), V \in K\left(R^{l}\right), \quad m, l-$ натуральные числа. Допустимые управления игроков $u(\tau), u: R_{+} \rightarrow U$, и v( $\left.\tau\right), v: R_{+} \rightarrow V-$ это измеримые функции времени.

Задано терминальное множество $M^{*}$ цилиндрического вида: $M^{*}=M_{0}-M$, где $M_{0}$ - линейное подпространство в $R^{n}$, а $M$ - компакт из ортогонального дополнения $L$ к $M_{0}$ в $R^{n} M \in K(L)$.

Первый игрок $(u)$ стремится вывести траекторию процесса на терминальное множество за кратчайшее время, а второй (v) - максимально оттянуть этот момент.

Указанный выше пример конфликтно-управляемого процесса имеет подробное описание и подходит для многих систем. Однако, он сложно 
применим для современной авиации, также, как и работы Н.Н. Красовского, так как описывает линейные системы и процессы, в то время, как полет ВК и конфликтная ситуация являются динамическими нелинейными процессами. К тому же, не учтены здесь и особенности распределенной среды, в которой находятся ВК, влияние внешних факторов и возмущений, математическая модель самого ВК.

Несмотря на значительные результаты в области автоматического управления движений комплексов летательных аппаратов, актуальными остаются работы по формированию алгоритмов управления, разработке структуры и исследованию динамических систем управления этими комплексами. Объектом управления здесь является строй летательных аппаратов, имеющий вид потока одиночных объектов или звеньев. При полете самолетов строем одной из наиболее важных проблем является проблема безопасности (предотвращения опасных сближений) взаимодействующих объектов группы.

В силу ряда причин задача автоматического управления полетом воздушных судов в групповом порядке представляет собой одну из наиболее сложных и специфических научно-технических проблем авиации, требующую комплексного решения. Характерной особенностью управления группой летательных аппаратов является нестационарность параметров полета. Вопросами автоматизации управления полетом строя активно занимались В.А. Боднер, А.А. Красовский, Ю.П. Доброленский, В.Г. Тарасов, С.В. Петров. В работах этих ученых были сформулированы общие требования к системам автоматического управления группой самолетов, представлено математическое описание строя как объекта управления, проведены исследования задачи управления самолетами в группе, даны технические предложения по построению систем управления строем самолетов.

В работе В.А. Боднера [9] подробно рассматривается вопрос решения конфликтных ситуаций ВК с позиции управления строем самолетов. Приводится подробная математическая модель строя самолетов, на основе которой можно сделать вывод, что непосредственное управление строем, учет интервала между самолетами, учет математической модели каждого из самолетов, определение ведущего и ведомых самолетов, соблюдение равенства скоростей и ускорений, высоты и курса - все это очень близко к проблеме расхождения ВК в воздухе и необходимости соблюдения норм эшелонирования. Однако, создание системы решения конфликтных ситуаций на основании управления полетом строя самолетов не получило своего развития из-за технических и принципиальных трудностей ее создания: необходимости учета нелинейных динамических нестационарных условий полета, существенных недостатков современных систем обмена данными между ВК.

Руфус Айзекс в своей работе, посвященной теории дифференциальных игр [10], очень подробно и интересно рассматривает задачи преследования, что соответствует и конфликтам ВК. Им подробно рассматриваются различные виды преследований, подходы к решению этих задач, критерии оптимальности их выполнения. Стратегии решения задачи преследования Айзекс разделяет на дискретные, аналитические и геометрические. Отдельно (C) В.В. Павлов, А.Е. Волков, Д.А. Волошенюк, 2015 
им ставится задача преследования как задача качества с некоторым критерием оптимальности. Работу Айзекса можно считать основополагающей в теории дифференциальных игр, как и его задачу преследования-убегания, которая является одним из примеров постановки задачи решения конфликтной ситуации ВК.

Необходимо помнить и о том, что поскольку в контуре управления ВК участвует пилот, то в качестве основы для разрешения навигационных конфликтов целесообразно использовать теорию технических эргатических организмов. Вопросы конфликтов в технических эргатических системах очень подробно рассмотрены в работе В.В. Павлова [11]. Там же приводится и подробное описание понятия функционального гомеостазиса.

В настоящее время существует много публикаций, посвященных исследованию процессов управления сближением конфликтующих подвижных объектов. При этом в большинстве известных работ рассматривается такая ситуация, когда о положении подвижных объектов и их управлении имеется полная информация. Значительно меньшее число работ посвящено рассмотрению тех случаев, когда измерения координат объектов управления производятся при наличии помех, относительно которых предполагается их стохастическая природа с известными вероятностными характеристиками.

В работе В.М.Кунцевича [12] делается акцент на случаи управления сближением конфликтующих объектов, когда определение фазовых координат объектов управления осуществляется с учетом стохастической природы имеющихся помех. Рассматривается также случай наличия неконтролируемых помех, свойства которых зависят от фазовых координат, что характерно для подвижных объектов в трехмерном пространстве. Автором рассматривается подход к решению проблемы, основанный на минимальных инвариантных множествах динамической системы, подверженных воздействию аддитивных ограниченных возмущений. Показано и доказано, что учет помех приводит к увеличению окрестности фазовых координат и, соответственно, - к возникновению зон неопределенностей положения объекта в пространстве.

В процессе решения задач по модернизации современных систем УВД и повышению безопасности полетов необходимо уделять достаточное внимание и вопросам, в которых рассматривается использование математических моделей для планирования, оптимизации и корректировки полета, а также систем устранения конфликтных ситуаций на земле и воздухе, учитывающих необходимость обеспечения вихревой безопасности [13].

Необходимо помнить, что после посадки самолета требуется время для затухания образовавшихся вихрей и зон турбулентности (рис. 1.). Это вызывает время ожидания, которое к тому же зависит от многих метеоусловий: наличия поперечных воздушных потоков, атмосферной турбулентности, температуры воздуха и т.д. Желание минимизировать задержки и увеличить пропускную способность упирается в проблему возникновения вихрей и спутного следа от приземляющихся самолетов. 


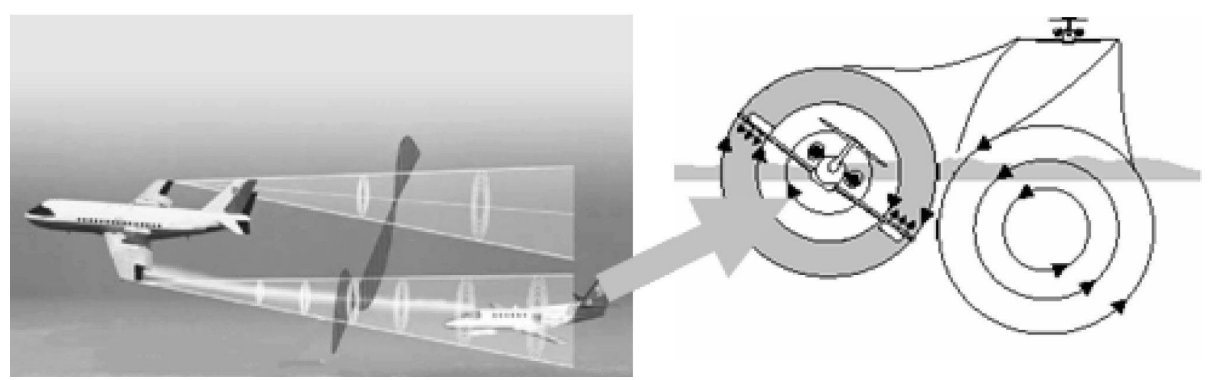

Рис. 1. Модель вихря, образующегося за летящим самолетом

Одним из важных направлений исследований в области организации УВД является целесообразность применения регулярной сетки для структурирования воздушных потоков (рис. 2 и 3) [13]. Регулярная сетка совмещает в себе две идеи: повторяющуюся структуру воздушных потоков и наличие выделенных слоев с параллельными маршрутами следования.

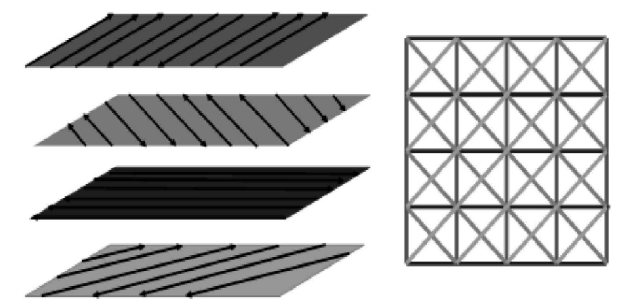

Рис. 2. Вертикально расположенные слои регулярной сетки и её горизонтальная структура

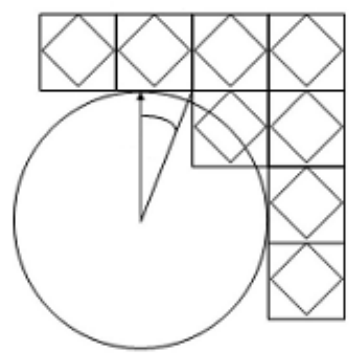

Рис. 3. Процесс присоединения самолета к регулярной сетке

Одним из обобщенных методов повышения безопасности полетов за счет уменьшения рисков конфликтных ситуаций является внедрение и применение концепции зональной навигации [14]. Суть зональной навигации в следующем. Применение все более сложного бортового навигационного оборудования, навигационных средств космического базирования и автономных инерциальных навигационных систем предполагает возможность осуществления выгодной замены полета от одного навигационного средства к другому на протяжении постоянных маршрутов по такой форме, по которой выполняется полет ВК по наиболее прямому маршруту между пунктами вылета и назначения. Зональная навигация предусматривает траекторию планового полета, которую можно задавать и в горизонтальной плоскости в виде маршрута, и в вертикальной - через высоты прохождения пунктов маршрута, углов и градиентов наклона (C) В.В. Павлов, А.Е. Волков, Д.А. Волошенюк, 2015 
траектории. Кроме того, можно задавать пространственно-временную траекторию, а не время прохождения некоторых пунктов.

Проблема внедрения зональной навигации состоит в том, что необходимо обеспечить полет по свободным траекториям с высокой точностью ее соблюдения и соответствием установленным в конкретном регионе требованиям. Соответственно, возникает проблема нормативного урегулирования и технического обеспечения полетов по зональной навигации, что требует больших материальных затрат.

Проведенный анализ последних исследований позволяет сделать следующие выводы.

- Проблемой всех методов и алгоритмов обнаружения и предотвращения конфликтов является неопределенность прогнозируемого положения самолетов, обусловленная погрешностями оценки текущей воздушной обстановки, неадекватностью математических моделей, описывающих движение самолета, влиянием ветра, навигационными погрешностями, ошибками управления, а также непредсказуемыми изменениями траектории, связанными с коррекцией движения пилотом или наземной системой УВД.

- Время прогноза является важнейшим фактором в системах обнаружения и предотвращения конфликтов. С одной стороны, с увеличением времени прогноза резко снижается достоверность оценки ситуации. С другой стороны, с уменьшением времени прогноза сокращается время принятия решения и ограничивается количество возможных вариантов устранения конфликта. Рассмотренные методы характеризуются большими временными затратами, что не решает проблемы повышения безопасности.

- При разработке методов обнаружения и оценки конфликтов естественным является желание получить аналитические выражения для оценки характеристик алгоритмов. Аналитические выражения позволяют более точно оценивать вероятность конфликта. Рассмотренные подходы и методы решения задачи обнаружения конфликтов достаточно сложные и для получения аналитического решения нужно сделать существенные упрощения, чтобы записать выражения, позволяющие произвести их алгоритмизацию и компьютерную реализацию. Эти методы не дают адекватного математического описания для прогнозирования развития неопределенности будущего положения самолетов на участках маневрирования.

- Значительное количество методов и алгоритмов решения конфликтных ситуаций рассчитаны на участие только двух ВК, а для более сложных случаев рассматриваются варианты парного перебора. При этом в парном переборе не учитывается влияние решения одного конфликта на другие.

- Основной проблемой рассмотренных работ является то, что они рассматривают стохастические подходы к решению задачи определения конфликтов, рассматривают только оценку их вероятности и не решают непосредственно задачу предотвращения конфликтов. 
Именно эти вышеперечисленные проблемы и недоставки и являются нерешенной частью общей проблемы, для решения которой необходимо искать новые подходы и методы в области систем и процессов управления.

\section{ИНВАРИАНТНАЯ СЕТЕЦЕНТРИЧЕСКАЯ СИСТЕМА УПРАВЛЕНИЯ КОНФЛИКТНЫМИ СИТУ АЦИЯМИ ВОЗДУШНЫХ КОРАБЛЕЙ}

Опишем подробно современный процесс обнаружения конфликта и его разрешения с предлагаемыми изменениями для реализации новой системы управления. Процессы обнаружения конфликта и его разрешения, как правило, разделены. Сигнал обнаружения является стартовым для начала работы алгоритма решения конфликта.

Универсальный алгоритм определения наличия конфликтной ситуации показан в работе [3]. Нами предлагается дополнить его алгоритмом определения приоритетов ВК в процессе конфликта. Система приоритетов позволяет ускорить процесс поиска оптимального маневра, поскольку содержит предварительно оцененное значение оптимизационного функционала для данного маневра. Такой алгоритм определения «пассивного/активного» (или «приоритетного») ВК для выполнения маневра представлен на рисунке 4.

Далее следует непосредственно процесс работы алгоритма решения конфликтной ситуации ВК и избегания опасного сближения (рис. 5).

При определении параметров необходимых маневров следует использовать построение математических функций областей управляемости каждого ВК для определения его возможностей. Решение этой задачи основывается на построении графиков зависимостей управляющих характеристик ВК друг от друга. Используется математический аппарат кинематики и динамики полета ВК [15] с учетом аэродинамических характеристик каждого ВК. Приведя результаты к выводу сил путем взятия производных, получим:

$$
\begin{aligned}
& \ddot{L}=F_{1}(\theta, \psi, m, P, \alpha,, \beta, X, \gamma, Y, Z, \delta)= \\
& =F_{1}\left(t,(H, L, Z, m(t)),(\theta, \psi, \delta)^{u^{\prime}},(\alpha, \beta, \gamma)^{u^{\prime \prime}},\left(\delta_{\text {pred }}, \delta_{z a k}\right)^{u^{\prime \prime \prime}},\left(V_{1}, V_{2}, V_{3}\right) ;\right. \\
& \ddot{H}=F_{2}(\theta, m, P, \alpha,, \beta, X, \gamma, Y, Z, \delta)= \\
& =F_{2}\left(t,(H, L, Z, m(t)),(\theta, \delta)^{u^{\prime}},(\alpha, \beta, \gamma)^{u "},\left(\delta_{\text {pred }}, \delta_{z a k}\right)^{u^{\prime \prime \prime}},\left(V_{1}, V_{2}, V_{3}\right) ;\right. \\
& \ddot{Z}=F_{3}(\theta, \psi, m, P, \alpha,, \beta, X, \gamma, Y, Z, \delta)= \\
& =F_{3}\left(t,(H, L, Z, m(t)),(\theta, \psi, \delta)^{u^{\prime}},(\alpha, \beta, \gamma)^{u^{\prime \prime}},\left(\delta_{\text {pred }}, \delta_{z a k}\right)^{u^{\prime \prime \prime}},\left(V_{1}, V_{2}, V_{3}\right),\right.
\end{aligned}
$$

где $(\theta, \psi, \delta)^{u^{\prime}}$ — параметры элементов траекторного управления; $(\alpha, \beta, \gamma)^{u^{\prime \prime}}$ параметры элементов кинематического управления; $\left(\delta_{\text {pred }}, \delta_{z a k}\right)^{u^{\prime \prime}}-$ параметры элементов аэродинамического управления.

Подставив аэродинамические данные конкретных ВК и построив функции зависимостей управляющих характеристик, получим графики, пример которых приведен на рисунках 6 и 7. 


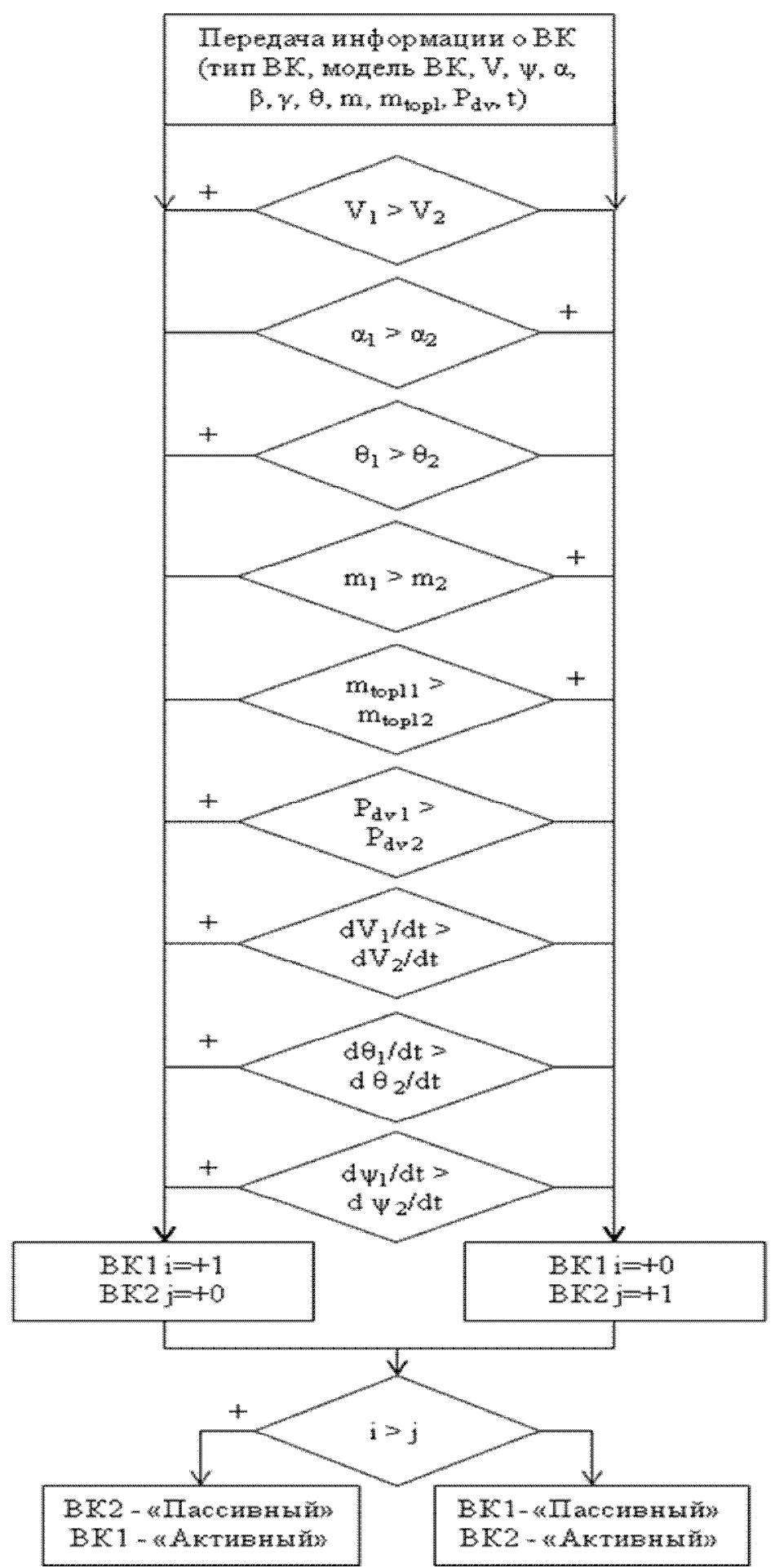

Рис. 4. Алгоритм определения «пассивного/активного» ВК 


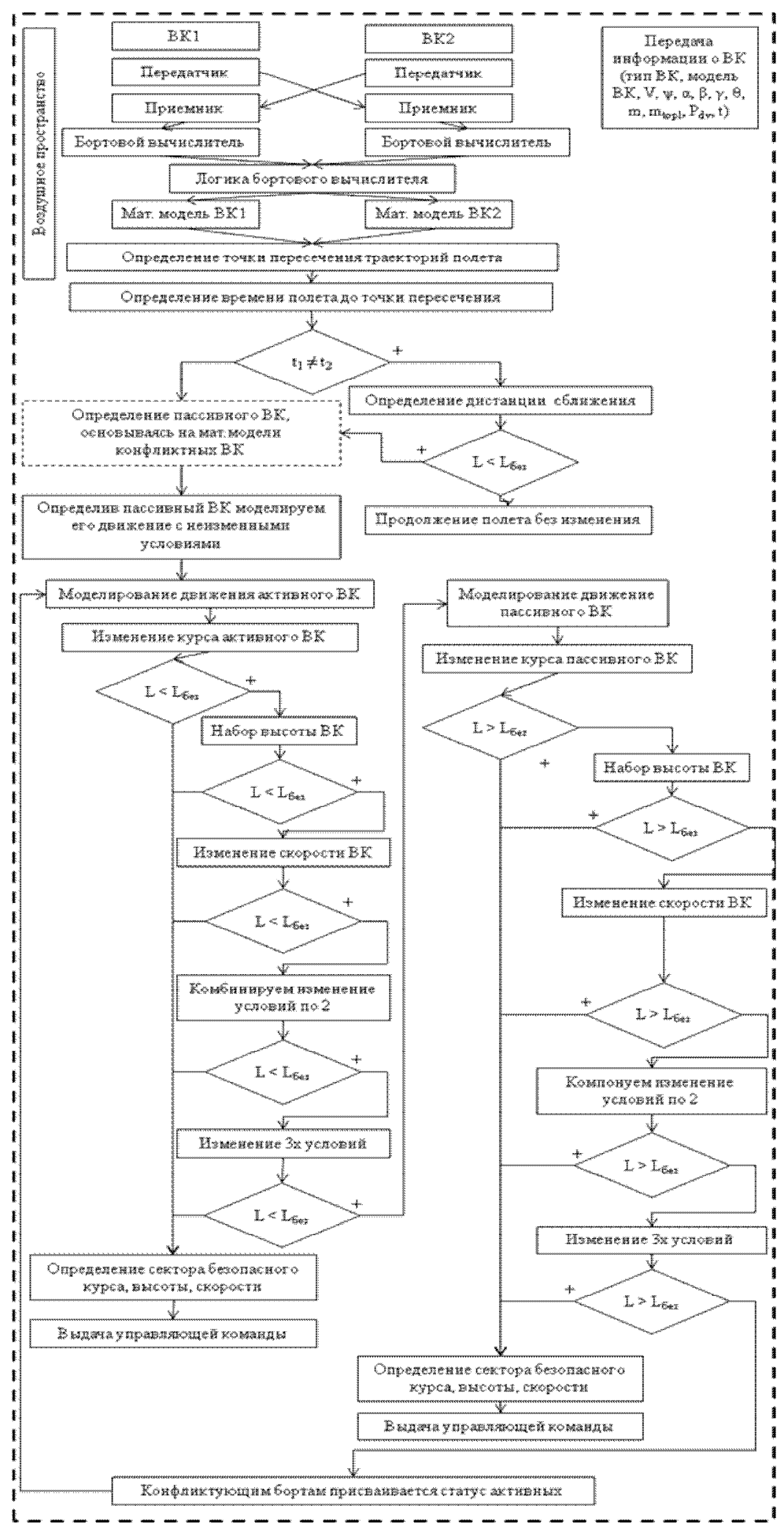

Рис. 5. Алгоритм решения конфликтной ситуации ВК и избегания опасного сближения

(C) В.В. Павлов, А.Е. Волков, Д.А. Волошенюк, 2015 


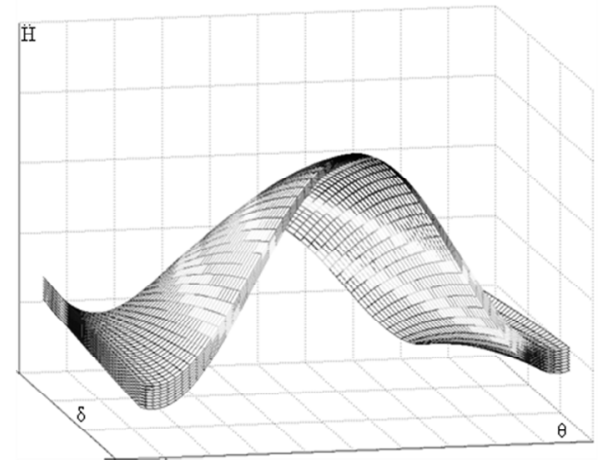

Рис. 6. Влияние параметров траекторного управления ВК на вызываемое ускорение

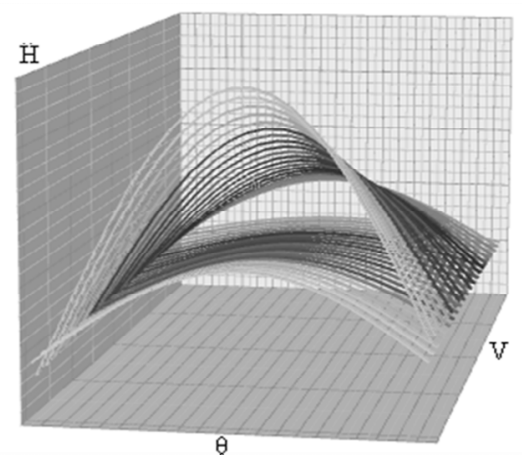

Рис. 7. Область управляемости ускорением ВК в зависимости от скорости и наклона траектории

Необходимо отметить, что современная система управления воздушным движением, является управляемой динамической системой, информация о ее состоянии периодически уточняется и обновляется. Траектория движения управляемых объектов - самолетов, является непрерывной и математически описывается непрерывными уравнениями динамики. Анализ воздушной обстановки, которая составляется и прогнозируется, а также принятие решения по предотвращению прогнозируемого конфликта, выполняются в дискретные моменты времени. Таким образом, задача предотвращения конфликтов имеет гибридную структуру, в которой дискретно-временной процесс анализа и принятия решений соединен с непрерывной динамикой процесса реализации решения. Такая гибридная структура должна производить команды, которые могут быть реализованы бортовой системой управления полетом, основанной на сетецентрических технологиях управления.

Поэтому, новая концепции УВД и предлагаемая технология управления конфликтными ситуациями предполагают применение современных технологий связи, навигации, наблюдения (CNS), позволяющих обеспечить самолет точной навигационной информацией глобальной системы спутниковой навигации (GNSS), а также наличие информационной связи с ближайшими самолетами с использованием автоматического независимого наблюдения в режиме радиопередачи и цифрового канала передачи данных 
(рис. 8) [16]. Особенностями такой современной системы является применение высокоскоростных линий передачи данных со специальным видом модуляции, широкое применение глобальной системы спутниковой навигации на всех стадиях полета, гибкое, скоординированное использование воздушного пространства с учетом всех участников и унификация систем обработки данных с целью последующей интеграции в региональные и глобальные сети. Это позволит повысить точность траекторной оценки, осуществить достоверное прогнозирование потенциально конфликтных ситуаций на время от 10 до 30 минут, что даст возможность реализовать стратегию кооперативного предотвращения конфликта с применением критериев оптимальности, которые учитывают движение всех конфликтующих самолетов.

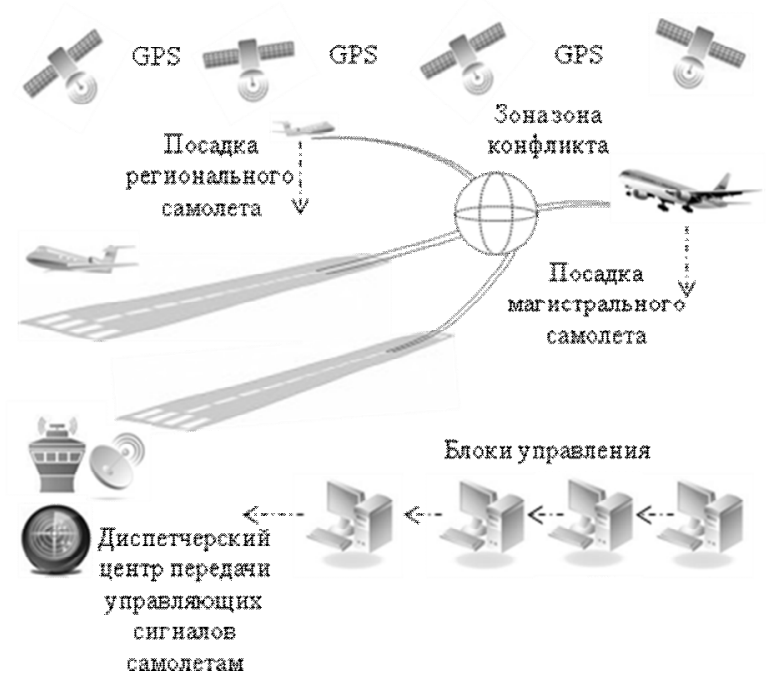

Рис. 8. Обобщенная структура сетецентрической системы управления конфликтами

Такая реализованная система управления в итоге получит название сетецентрической системы управления, так как будет иметь интеллектуальную распределенную структуру (рис. 9).

\section{МАТЕМАТИЧЕСКОЕ ОПИСАНИЕ ПРОЦЕССА ОПРЕДЕЛЕНИЯ И ПРЕДОТВРАЩЕНИЯ ОПАСНОГО СБЛИЖЕНИЯ ВК}

Вероятность конфликта определяется как вероятность того, что расстояние между двумя самолетами станет меньше некоторого установленного порога. Задача системы предотвращения конфликтов состоит в том, чтобы удерживать значение вероятности конфликта ниже некоторого допустимого уровня.

Математическое описание процесса определения и предотвращения опасного сближения ВК выглядит следующим образом $[2,3,9,15]$. 


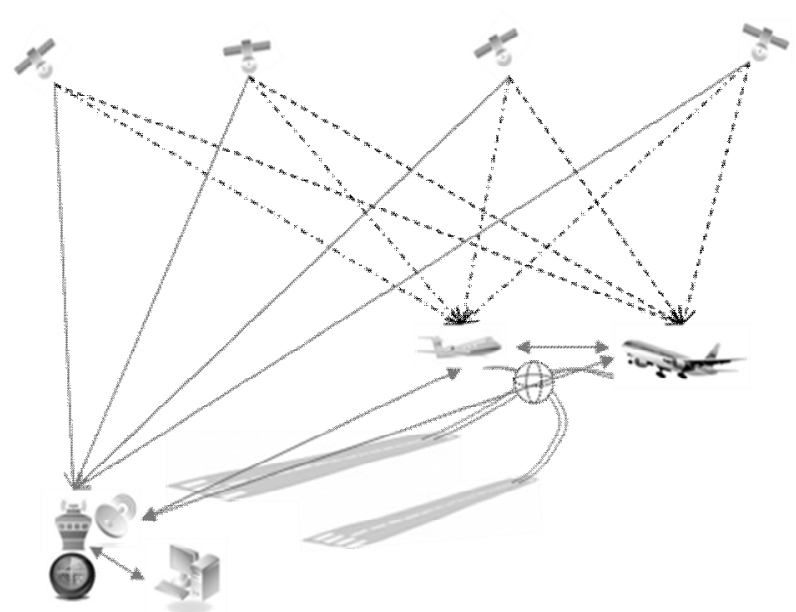

Рис. 9. Линии связи в структуре сетецентрической системы управления конфликтными ситуациями

В общем виде, если движение двух ВК в пространстве задано векторами скоростей $\bar{V}_{1}$ и $\bar{V}_{2}$, то максимальное сближение самолетов (расстояние расхождения) определяют из выражения:

$$
r_{\min }=\bar{V}_{12} * \bar{d} / V_{12},
$$

где $\bar{V}_{12}=\bar{V}_{1}-\overline{V_{2}}$ - вектор относительной скорости; $\bar{d}$ - вектор расстояния между самолетами.

Время достижения максимального сближения определяется:

$$
t_{\min }=1 / \bar{V}_{12} * \sqrt{d^{2}-r_{\min }^{2}} .
$$

Расстояние между самолетами:

$$
d(t)=\left(V_{\text {omt }}^{2} t^{2}-2 d_{0} V_{c \sigma . t} t+d_{0}^{2}\right)^{1 / 2},
$$

где $V_{\text {отн }}=\left(V_{1}^{2}-2 V_{1} V_{2} \cos \varphi+V_{2}^{2}\right)^{1 / 2}-$ относительная скорость самолетов; $V_{c б л}=V_{1} \cos \theta_{1}-V_{2} \cos \left(\theta_{1}+\varphi\right)-$ скорость сближения самолетов; $\theta_{1}-$ пеленг второго самолета относительно направления полета первого; $\varphi-$ относительный курс; $d_{0}-$ расстояние между самолетами в начальный момент времени.

Время до наибольшего сближения:

$$
t_{c б л}=d_{0} V_{\text {сбл }} / V_{\text {отн }}^{2} \text {. }
$$

Расстояние между самолетами $d_{c б, l}$ в момент наибольшего сближения:

$$
d_{\text {сбл }}=d_{0} V_{H} / V_{\text {отн }}{ }^{1 / 2} \text {, }
$$

где $V_{H}=V_{2} \sin \left(\theta_{1}+\varphi\right)-V_{1} \sin \theta_{1}-$ составная относитель-ной скорости, направленная перпендикулярно к линии, которая соединяет самолеты.

В случае пересечения трас полета двух самолетов, и если известны их координаты, то расстояние между самолетами можно определить:

$$
L_{12}=\sqrt{\left[x_{1}(t)-x_{2}(t)\right]^{2}+\left[y_{1}(t)-y_{2}(t)\right]^{2}},
$$

где $x$ и $y$ - координаты самолетов. 


\section{МАТЕМАТИЧЕСКАЯ ПОСТАНОВКА ЗАДАЧИ РЕШЕНИЯ КОНФЛИКТНОЙ СИТУАЦИИ САМОЛЕТОВ НА ЭТАПЕ ПОСАДКИ}

Пусть в начальный момент времени $t_{0}$ в некоторой зоне полетов ограниченного размера $Q$ находится множество из $n$ самолетов:

$$
P=\left\{P_{1}, P_{2}, P_{3}, \ldots, P_{n}\right\} .
$$

Будем считать, что в начальный момент времени $t_{0}$ положение всех самолетов из множества $P$ определяется случайным образом, но так, чтобы между самолетами не возникало конфликтных ситуаций на интервале времени $\left(t_{0}, t_{b}\right)$, где $t_{b}-$ некоторый момент буферного времени. Также предположим, ни один из самолетов из множества $P$, двигаясь по плану, не успеет выйти за границы зоны полетов, и ни один из самолетов не зайдет в зону полетов извне на промежутке времени $\left(t_{0}, t_{g}\right)$, где $t_{g}-$ некоторый граничный момент времени, на который прогнозируется траектория каждого самолета. Значит, множество $P$ можно считать неизменным на интервале времени $\left(t_{0}, t_{g}\right)$. Допустим, что методы и алгоритмы определения конфликтной ситуации являются известными. Тогда пусть существует достаточно большое количество обнаруженных конфликтных пар самолетов $P_{i}$ и $P_{j}$ на промежутке времени $\left(t_{b}, t_{g}\right)$, для которых не выполняется условие безопасного эшелонирования:

$$
L\left(P_{i}, P_{j}, t\right) \geq L_{n}, t \in\left(t_{b}, t_{g}\right),
$$

где $L\left(P_{i}, P_{j}, t\right)$ - расстояние между самолетами $P_{i}$ и $P_{j}$ в момент времени $t$; $L_{n}$ - норма безопасного эшелонирования самолетов.

Кроме того, предположим, что плотность самолетов в зоне полета сравнительно большая, поэтому ситуацию можно рассматривать как групповую конфликтную ситуацию.

Решение конфликтной ситуации заключается в определении для каждого конфликтующего самолета $P_{i} \in P$ таких траекторий, которые бы обеспечивали отсутствие пересечений со всеми другими самолетами из множества $P$ на интервале времени $\left(t_{b}, t_{g}\right)$. При этом полученное решение должно соответствовать глобальному оптимуму по критерию минимального отклонения самолетов от начальных траекторий.

Задачу определения оптимального маневрирования по устранению конфликтной ситуации можно сформулировать как задачу оптимального управления динамической системой. Оптимальность определяется как минимизация затрат при выполнении маневрирования по предотвращению потенциально конфликта при заданных ограничениях.

При оптимизации безопасных маневров используют следующие основные критерии: различные меры пространственного отклонения от первоначальной траектории; минимум безопасного расстояния между конфликтующими ВК; временное отклонение от запланированного прибытия в точку; продолжительность маневра; степень отклонения управляющих скоростных и угловых параметров; расход топлива; приоритеты ВК; приоритеты типов маневров; комфорт пассажиров; сложность маневров.

Глобальный оптимум по критерию минимального отклонения самолетов от начальных траекторий - это один из основных критериев оптимальности варианта решения конфликтной ситуации $[3,4]$. В его качестве выступает (C) В.В. Павлов, А.Е. Волков, Д.А. Волошенюк, 2015 
показатель, который характеризует отклонения конфликтующих самолетов от начальных планов полета, а именно, площадь необходимого маневра

$$
S=\int_{x_{0}}^{x_{k}}\left|y_{0}(x)-y_{k}(x)\right| d x,
$$

где $y_{k}(x), y_{0}(x)$ - начальные и маневровые линии пути самолета на плоскости, $x_{0}, x_{k}$ - координаты точки начала и конца маневра. Площадь маневра учитывает временные и пространственные потери на выполнение маневра, но не учитывает временную разницу с планом после завершения маневра.

Решение игровой задачи уклонения-преследования предлагается решать и с точки зрения теории инвариантности [17]. Необходимо определять стратегию преследуемого игрока $\left(P_{i}\right)$ таким образом, чтобы состояние дифференциальной игры было инвариантным к стратегии игрокапреследователя $\left(P_{j}\right)$. При этом качество изменения инвариантного состояния стратегии преследуемого игрока $\left(P_{i}\right)$ и дифференциальной игры в целом должно быть оптимальным с точки зрения минимизации некоторых условных критериев (например, глобального оптимума).

В работе [17] показан пример решения такой задачи с помощью теории нелинейной инвариантности и доказана рациональность и перспективность такого подхода. Существенным преимуществом является то, что такое решение основывается на применении в нелинейных динамических системах в распределенной среде, каковым и является самолет в целом. Отметим также, что использование для синтеза эргатической системы управления движением ВК метода нелинейной интегральной инвариантности позволяет обеспечить наибольшие возможности для проявления свойств функционального гомеостазиса.

Соответственно, вариант решения поставленной задачи решения конфликтной ситуации самолетов с помощью теории инвариантности будет звучать так: для существования решения данной дифференциальной игры уклонения-преследования (решения конфликтной ситуации самолетов) в некоторой области необходимо и достаточно, чтобы для параметра управления динамическим процессом изменения инвариантного состояния дифференциальной игры возможно было выбрать такое значение, которое обеспечит существование функции Ляпунова для такой замкнутой системы.

Проведено соответствующее моделирование, позволяющее проанализировать такой подход к решению задачи конфликтной ситуации ВК (рис. 10).

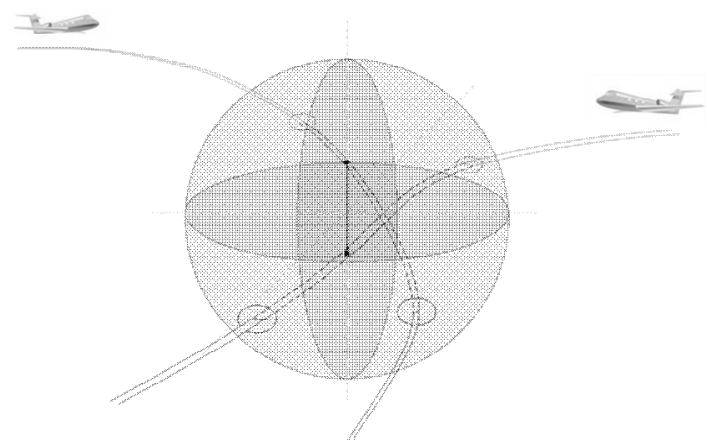

Рис. 10. Зона возникновения конфликтной ситуации ВК на этапе посадки 
Результаты моделирования и итогового расхождения воздушных кораблей при возникшей угрозе столкновения с помощью новой сетецентрической системы управления конфликтными ситуациями, с учетом глобального оптимума, основанной на теории инвариантности, показали очень обнадеживающие и перспективные результаты (рис. 11).

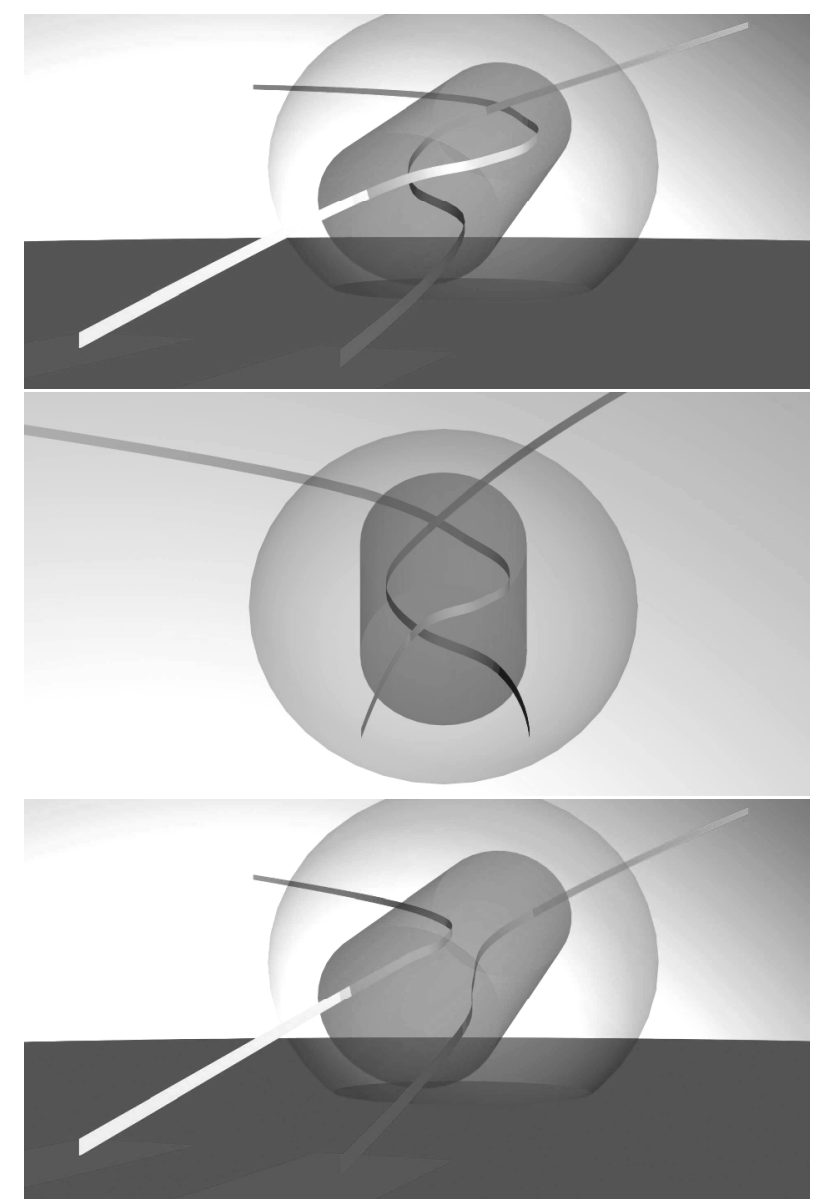

Рис. 11. Модели области зоны конфликтной ситуации ВК с её успешным решением

\section{Выводы}

Рассматривая процесс конфликта воздушных кораблей через призму дифференциальных игр, можно сделать вывод, что конструктивное аналитическое решение удается построить лишь для игр с двумя убегающими. Для большего числа убегающих оптимальное поведение игроков находится только численно. Но и численное исследование возможно только для количества игроков не более пяти, иначе задачи уже трудноразрешимы. Сложность этих задач становится очевидной, если учесть тот факт, что принятие решений в них должно происходить в режиме реального времени. Следовательно, методы и алгоритмы, реализующие принятие решений, должны быть весьма быстрыми. 
Движение самолета является управляемым процессом, поэтому параметры траектории его полета коррелированы во времени. Такой подход позволяет более адекватно учитывать динамику полета при прогнозировании траектории на участках наиболее опасного сближения самолетов. Корреляционная зависимость позволяет получить более достоверный прогноз траектории полета.

Большинство используемых сейчас методов решения конфликтной ситуации, ее моделирования и решения описывают только линейные и стационарные системы, в то время как полет самолета и конфликт ВК является динамическим нелинейным процессом.

Основной проблемой при разработке систем предотвращения конфликтной ситуации является достоверное прогнозирование воздушной обстановки, которое зависит, в первую очередь, от степени адекватности принятой математической модели движения самолета реальному процессу воздушного движения. При прогнозировании случайного процесса основными факторами, влияющими на неопределенность будущего положения самолета, считаются ошибки учета ветра и его изменчивость, навигационные ошибки, ошибки пилотирования и системы управления полетом, непредвиденные перепланировки маршрута полета. Особо следует отметить неопределенность будущего положения самолета из-за ошибок моделирования процесса его полета. Причиной ошибки может быть несоответствие принятых предположений реальным условиям полета или неопределенность значений параметров принятой модели. В некоторых случаях математическая модель не может быть решена аналитически. Тогда решение находится численным приближением, ошибка которого также вносит свою долю неопределенности. Соответственно, это вызывает вопрос о внесении в модель конфликтно-управляемого процесса не только детерминированной, но и стохастической неопределенности.

Наибольшая эффективность решения конфликтов достигается при использовании распределенных наземно-бортовых систем. К сожалению, на данный момент такие системы полноценно не реализованы. В данной работе предлагается структура такой системы.

Несмотря на сравнительную простоту необходимого тактического маневра во взаимодействиях конфликтующих ВК, математическое решение задачи отыскания соответствующих оптимальных траекторий представляет собой достаточно сложную задачу. Ее специфика заключается в неоднозначности искомых траекторий, в том числе из-за немонотонности поведения параметров целевых объектов, и поэтому освещение рассматриваемого вопроса приобретает смысл поиска общего подхода к получению точных решений в зависимости от конкретных требований.

Важным недостатком современных систем обнаружения конфликта ВК является то, что в модели прогнозируемого движения ВК не учитывается неопределенность, связанная с двумя главными обстоятельствами: погрешностями в определении текущего состояния ВК и неопределенностью в модели поведения ВК на прогнозируемом интервале времени. Именно поэтому вокруг ВК необходимо строить виртуальную защитную зону неопределенности в виде сферической области. И в данном случае 
конфликтом будет считаться уже именно нарушение границ этой зоны зоной другого ВК.

В условиях наличия обнаруженных конфликтов алгоритм решения конфликтных ситуаций ВК должен формировать рекомендации, которые должны обеспечить, в общем случае, пространственный маневр уклонения с целью предотвращения возможного опасного сближения ВК, а после разрешения конфликта — обеспечить возвращение ВК на плановую траекторию и ее дальнейшее выдерживание. При этом в процессе выдачи рекомендаций по выполнению маневра должны учитываться критерии оптимальности.

Это перспективная задача, требующая своего решения. В данной работе предложены новые подходы к решению этой задачи.

1. Евроконтроль. Аэрокосмическая стратегия для участников ЕКГА. — ИКАО: 2001. — $91 \mathrm{c}$.

2. Харченко В.П. Риски столкновения и эшелонирование воздушных кораблей / В.П. Харченко, Г.Ф. Аргунов, С.А. Закора и др. - К.: НАУ, 2011. - 326 с.

3. Закора С.А. Анализ методов решения конфликтных ситуаций в условиях свободного полета / С.А. Закора. - Вестник Национального авиационного университета, 2005, № $1 .-$ C. $42-74$.

4. Глобальный аэронавигационный план ИКАО на 2013-2028 года. — Канада, 2013.

5. Красовский Н.Н. Позиционные дифференциальные игры / Н.Н. Красовский, А.И. Субботин. - М.: Наука, 1974. — 458 с.

6. Красовский Н.Н. Игровые задачи о встрече движений / Н.Н. Красовский. - М.: Наука, 1970. - $424 \mathrm{c}$.

7. Чикрий А.А. Гарантированный результат в игровых задачах управления движением / А.А. Чикрий. - М.: Труды института математики РАН, 2010. - С. 223-232.

8. Пшеничный Б.Н. Задача об уклонении от встречи в дифференциальных играх / Б.Н. Пшеничный, А.А.Чикрий. - М.: Журнал вычислительной математики и физики, 1974, № 6. - С. 1416-1426.

9. Боднер В.А. Системы управления летательными аппаратами / В.А. Боднер. - М.: Машиностроение, 1973. - $501 \mathrm{c}$.

10. Айзекс Р. Дифференциальные игры / Руфус Айзекс. - М.: Мир, 1967. — 480 с.

11. Павлов В.В. Конфликты в технических системах / В.В. Павлов. - К.: Вища школа, 1982. $-183 \mathrm{c}$.

12. Кунцевич В.М. Оптимальное управление сближением конфликтующих подвижных объектов в условиях неопределенности / В.М. Кунцевич, А.В. Кунцевич. - К.: Кибернетика и системный анализ, 2002, №2. - С. 95-104.

13. Золотухин В.В. Некоторые актуальные задачи управления воздушным движением / В.В. Золотухин, В.К. Исаев, Б.Х. Давидсон. - М.: Труды МФТИ, 2009, № 3. C. $94-114$.

14. Харченко В.П. Решение конфликтных ситуаций между воздушными кораблями маневрированием курса самолета / В.П. Харченко, Д.В. Васильев. - Вестник Национального авиационного университета, 2011, № 2. - С. 15-20.

15. Мхитарян А.М. Динамика полета / А.М. Мхитарян, П.С. Лазнюк, В.С. Максимов и др. - М.: Машиностроение, 1978. - 424 с.

16. Бочкарев В.В. Концепция и системы CNS/ATM в гражданской авиации / В.В. Бочкарев, В.Ф. Кравцов, Г.А. Крыжановский. - М.: Академкнига, 2003. - 415 с.

17. Павлов В.В. Инвариантность и автономность нелинейных систем управления / В.В. Павлов. - К.: Наукова думка, 1971. — 272 с. 
UDC 656.7.084

\title{
INVARIANT NET-CENTRIC CONTROL SYSTEM FOR CONFLICT AVOIDANCE OF AIRCRAFTS IN THE LANDING PHASE
}

\author{
V.V. Pavlov, A.E. Volkov, D.A. Voloshenyuk \\ International Research and Training Center for Information Technologies and \\ Systems of National Academy of Sciences of Ukraine and Ministry of Education and \\ Science of Ukraine
}

Introduction. The article discusses the question of the need to create a control system of conflict situations between the aircrafts in the landing phase.

The purpose of this research is to create a method and system of conflict resolution between the aircrafts on the route of flight, takeoff and landing phases with the condition to provide a high and guaranteed level of flight safety. The approach considered in the article will be based on the principles of using the network-centric technologies and the theory of invariance.

Results. The expected result of this work is to create a new universal control system of conflict situations between the aircrafts based on network-centric technologies and principles of the theory of invariance, which will meet all the requirements of modern air traffic management (ATM) to provide a guaranteed level of safety.

Conclusion. Summarizing the results it is shown that a new approach to the problem of creating a control system of conflict situations between the aircrafts based on research in the field of differential games and the theory of invariance is proposed.

Keywords: net-centric system, flight safety, invariance, conflict situations, differential games, free flight.

1. Eurocontrol. Airspace Strategy for the ECAC States. ICAO: 2001, 91 p. (in Russian).

2. Harchenko V.P., Argunov G.F, Zakora S.A. et al. The risks of collision and the flight level of aircrafts. K.: NAU, 2011, 326 p. (in Russian).

3. Zakora S.A. Classification of conflict resolution modeling methods for free flight. Bulletin of the National Aviation University, 2005, no. 1, pp. 42-74 (in Russian).

4. The ICAO Global Air Navigation Plan for 2013-2028 years. ICAO: Canada, 2013. (in Russian).

5. Krasovskiy N.N., Subbotin A.I. The positional differential games. Moskow: Science, 1974, 458 p. (in Russian).

6. Krasovskiy N.N. Game Problems of counter movements. Moskow: Science, 1970, 424 p. (in Russian).

7. Chikriy A.A. The guaranteed result in game problems of traffic control. M.: Proceedings of the RAS Institute of Mathematics, 2010, pp. 223-232. (in Russian).

8. Pshenichnyiy B.N., Chikriy A.A. The problem of collision avoidance in differential games. M.: Bulletin of Computational Mathematics and Physics, 1974, no. 6, pp. 1416-1426 (in Russian).

9. Bodner V.A. Aircraft Control System. M.: Mashinostroenie, 1973, 501 p. (in Russian).

10. Ayzeks R. The differential games. M.: Mir, 1967, 480 p. (in Russian).

11. Pavlov V.V. The conflicts in technical systems. K.: Vischa shkola, 1982, 183 p. (in Russian).

12. Kuntsevich V.M. Optimal control of convergence of conflicting moving objects under uncertainty. K.: Cybernetics and systems analysis, 2002, no. 2, pp. 95-104 (in Russian).

13. Zolotuhin V.V. Some actual problems of air traffic control. M.: Proceedings of MFTI, 2009, no. 3, pp. 94-114 (in Russian). 
14. Harchenko V.P. Aircraft conflicts resolution by course maneuvering. Bulletin of the National Aviation University, 2011, no. 2, pp. 15-20 (in Russian).

15. Mhitaryan A.M. Aircraft flight dynamics. M.: Mashinostroenie, 1978. 424 p. (in Russian).

16. Bochkarev V.V., Kravtsov V.F., Kryizhanovskiy G.A. The concept and systems of CNS/ATM in civil aviation. M.: Akademkniga, 2003, 415 p. (in Russian).

17. Pavlov V.V. The invariance and autonomy of nonlinear control systems. K.: Naukova Dumka, 1971, 272 p. (in Russian).

Получено 23.02.2015 


\title{
ОПТИМАЛЬНОЕ УПРАВЛЕНИЕ НЕСТАЦИОНАРНЫМ РЕЖИМОМ КАСКАДНОГО ТЕРМОЭЛЕКТРИЧЕСКОГО ОХЛАДИТЕЛЯ
}

\author{
М.П. Коцур ${ }^{1}$, А.Г. Наконечный ${ }^{2}$ \\ ${ }^{1}$ Черновицкий национальный университет имени Юрия Федьковича \\ ${ }^{2}$ Киевский национальный университет имени Тараса Шевченко
}

Предложен способ решения задачи оптимального управления объектом с распределенными параметрами, который описывается системой нелинейных дифференциальных уравнений параболического типа с нестандартными граничными условиями. Способ заключается в дискретизации объекта и переходе к объекту с сосредоточенными параметрами, для оптимизации которого применяется принцип максимума Понтрягина. Такой метод использован для оптимизации нестационарного процесса термоэлектрического охлаждения. Рассчитаны оптимальные зависимости тока питания каскадного термоэлектрического модуля от времени, которые обеспечивают минимальную температуру охлаждения.

Ключевые слова: объект с распределенными параметрами, оптимальное управление, принцип максимума Понтрягина, оптимизация, нестационарное термоэлектрическое охлаждение.

Запропоновано спосіб розв'язування задачі оптимального керування об'єктом 3 розподіленими параметрами, що описується системою нелінійних диференційних рівнянь параболічного типу 3 нестандартними крайовими умовами. Спосіб полягає у дискретизації об’єкту і переході до об'єкта 3 зосередженими параметрами, для оптимізації якого використовується принцип максимуму Понтрягіна. Такий метод застосовано для оптимізації нестаціонарного процесу термоелектричного охолодження. Розраховано оптимальні залежності струму живлення каскадного термоелектричного модуля від часу, які забезпечують мінімальну температуру охолодження.

Ключові слова: об'єкт з розподіленими параметрами, оптимальне керування, принцип максимуму Понтрягіна, оптимізація, нестаціонарне термоелектричне охолодження.

\section{ВВЕДЕНИЕ}

Развитие теории и методов оптимального управления объектами с распределенными параметрами, поведение которых описывается дифференциальными уравнениями в частных производных, весьма актуально для многих областей науки и техники. Многие явления и процессы в физике и технике моделируются как объекты с распределенными параметрами. Процессы нагрева и охлаждения тел [2-4], химической диффузии [5], явления деформации и вибрации [6,7], распространение акустических, световых, электромагнитных волн $[2,8]$, движение потоков жидкостей и газов, процессы квантовой механики $[9,10]$, газодинамики, горения, химических и ядерных реакций [11-14] — все это примеры объектов с распределенными 
параметрами, которые описываются функциями пространственных координат и времени. Эффективное функционирование таких объектов возможно при решении задач оптимального управления, результатами которых являются оптимальные функции управления.

Теория оптимального управления широко используется для оптимизации объектов с сосредоточенными параметрами, состояние которых описывается системами обыкновенных дифференциальных уравнений [15-18]. Для них сформулированы необходимые условия оптимальности, например, принцип максимума Понтрягина [19], на основании которых создаются алгоритмы и компьютерные методы поиска функций управлений, оптимизирующих объект.

Развитие теории оптимального управления для объектов с распределенными параметрами и методов определения их характеристик является более сложной проблемой по сравнению с аналогичной задачей для систем с сосредоточенными параметрами, так как приходится решать дифференциальные уравнения в частных производных, учитывая сложность реальных граничных условий, которые характеризуют поведение объекта. Граничные условия задачи могут содержать частные производные, функции управления и т.п. Необходимые условия оптимальности получены только для некоторых задач с распределенными параметрами с простыми граничными условиями [20-23]. Поэтому актуальной задачей является разработка теории оптимального управления для объектов, которые описываются системами дифференциальных уравнений в частных производных с нетривиальными граничными условиями.

Примером объекта с распределенными параметрами является нестационарный процесс термоэлектрического охлаждения. Самое глубокое охлаждение достигается при использовании каскадного охладителя, работающего в нестационарном режиме. В таких устройствах каждый последующий каскад охлаждает предыдущий, обеспечивая таким образом существенное снижение температуры. Анализ результатов научных исследований $[18,24]$ показывает, что задачи оптимизации такого объекта были решены только для простейших физических моделей термоэлектрического преобразователя, или вообще не рассматривались. Это объясняется сложностью математической модели нестационарного процесса каскадного охлаждения.

Цель работы - разработка метода оптимизации управления нестационарным режимом термоэлектрического охладителя путем построения метода оптимального управления и алгоритма численного решения соответствующей математической задачи оптимизации нестационарных одномерных процессов с распределенными параметрами.

\section{ПОСТАНОВКА ЗАДАЧИ ОПТИМАЛЬНОГО УПРАВЛЕНИЯ НЕСТАЦИОНАРНЫМ ПРОЦЕССОМ КАСКАДНОГО ТЕРМОЭЛЕКТРИЧЕСКОГО ОХЛАЖДЕНИЯ}

На примере нестационарного процесса каскадного термоэлектрического охлаждения рассмотрим постановку задачи оптимального управления (C) М.П. Коцур, А.Г. Наконечный, 2015 
объектом с распределенными параметрами, поведение которого описывается системой дифференциальных уравнений параболического типа с нестандартными граничными условиями. Объектом исследования является охлаждающий каскадный модуль (рис. 1a). Каждый каскад состоит из термоэлементов (рис. 1б), соединенных последовательно в электрическую цепь и параллельно по отношению к тепловому потоку. Каждый термоэлемент содержит две ветви из полупроводниковых материалов $n$ - и $p$-типов проводимости. При протекании электрического тока указанной на рис. $1 a$ полярности, спай 4 охлаждается вследствие поглощения тепла Пельтье, а противоположный спай нагревается. Для обеспечения более глубокого охлаждения каждый последующий каскад охлаждает предыдущий. Каскады отделены один от другого электроизоляционными пластинами из материала с высокой теплопроводностью. Как правило, электрическое соединение каскадов последовательное, поэтому сила тока в ветвях термоэлементов разных каскадов одинаковая. Охлаждаемый объект размещается на теплопоглощающей поверхности верхнего (холодного) каскада. Обычно путем интенсивного отвода тепла температура горячих спаев термоэлементов нижнего (горячего) каскада поддерживается на уровне температуры окружающей среды.

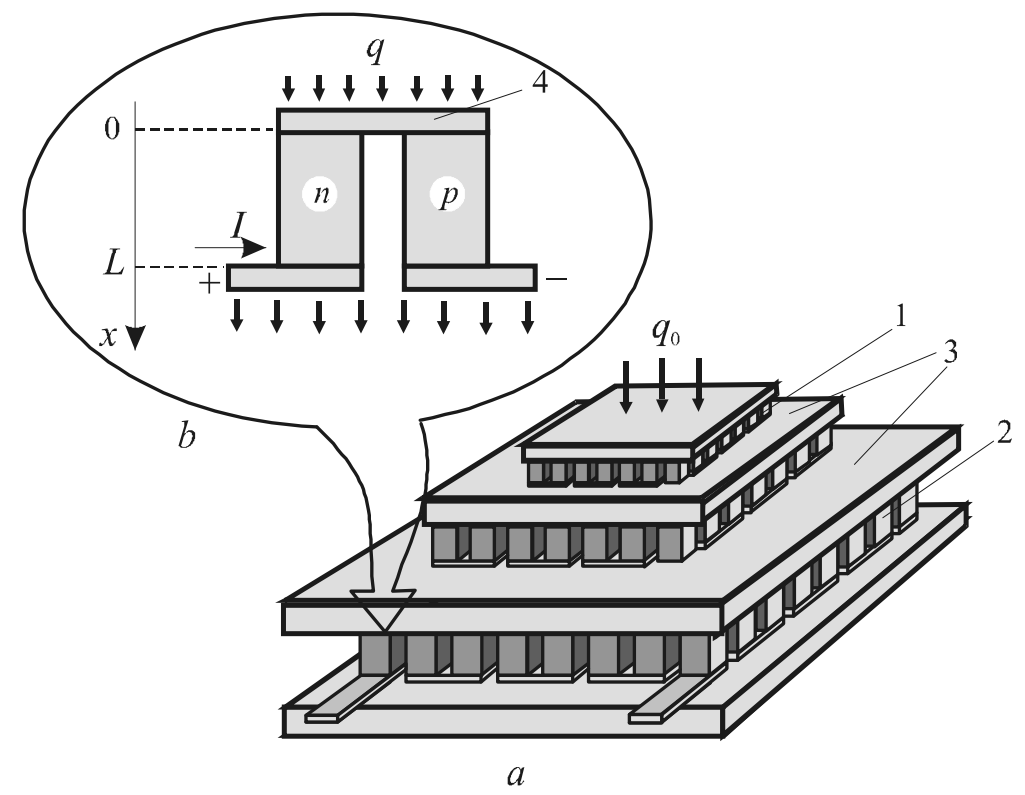

Рис. 1. Схема охлаждающего каскадного модуля $(a)$ и термоэлемента (б).

$1-1$-й (холодный) каскад, $2-K$-й (горячий) каскад, $3-$ электроизоляционные пластины, 4 - охлаждающий спай термоэлемента.

Нестационарный режим работы каскадного термоэлектрического охладителя описывается системой одномерных уравнений нестационарной теплопроводности в ветвях термоэлементов $n$ - и $p$-типов проводимости для $K$-каскадного устройства, которая имеет вид [18]: 


$$
\begin{aligned}
& \frac{\partial T_{n, p}^{(k)}}{\partial t}=\frac{1}{c_{n, p}}\left[\frac{\partial}{\partial x_{k}}\left(\kappa_{n, p} \frac{\partial T_{n, p}^{(k)}}{\partial x_{k}}\right)+\rho_{n, p} \frac{I^{2}}{s_{n, p}{ }^{2}}-T_{n, p}^{(k)} \frac{I}{s_{n, p}} \frac{\partial \alpha_{n, p}}{\partial T} \frac{\partial T_{n, p}^{(k)}}{\partial x_{k}}\right], \\
& k=1, \ldots, K
\end{aligned}
$$

где $T$ - температура, $I$ - ток питания ветвей термоэлементов. Начальные условия задачи следующие:

$$
T_{n, p}^{(k)}(x, 0)=T_{a},
$$

Совокупность граничных условий записывается следующим образом:

- условие теплового баланса для холодной поверхности первого каскада, на которой находится охлаждаемый объект с теплоемкостью $g_{1}$, выделяющий тепло $q_{0}$ :

$$
\begin{aligned}
\frac{\partial T_{n}^{(1)}(0, t)}{\partial t}= & \frac{n_{1}}{g_{1}}\left[q_{0}-K_{H}\left(s_{n}+s_{p}\right)\left(T_{n}^{(1)}-T_{a}\right)+\kappa_{n} s_{n} \frac{\partial T_{n}^{(1)}}{\partial x_{1}}-\alpha_{n} I T_{n}^{(1)}+\right. \\
& \left.+\kappa_{p} s_{p} \frac{\partial T_{p}^{(1)}}{\partial x_{1}}-\alpha_{p} I T_{p}^{(1)}+I^{2}\left(\frac{r_{0}}{s_{n}}+\frac{r_{0}}{s_{p}}\right)\right]_{x_{1}=0} ;
\end{aligned}
$$

- условия теплового баланса между каскадами:

$$
\begin{gathered}
\frac{\partial T_{n}^{(k+1)}(0, t)}{\partial t}= \\
=\frac{n_{k}}{g_{k+1}}\left[\left(\alpha_{n}+\alpha_{p}\right) I T_{n}^{(k)}-\kappa_{n} s_{n} \frac{\partial T_{n}^{(k)}}{\partial x_{k}}-\kappa_{p} s_{p} \frac{\partial T_{p}^{(k)}}{\partial x_{k}}+I^{2}\left(\frac{r_{0}}{s_{n}}+\frac{r_{0}}{s_{p}}\right)\right]_{x_{k}=L}- \\
-\frac{n_{k+1}}{g_{k+1}}\left[\left(\alpha_{n}+\alpha_{p}\right) I T_{n}^{(k+1)}-\kappa_{n} s_{n} \frac{\partial T_{n}^{(k+1)}}{\partial x_{k+1}}-\kappa_{p} s_{p} \frac{\partial T_{p}^{(k+1)}}{\partial x_{k+1}}-\right. \\
\left.-I^{2}\left(\frac{r_{0}}{s_{n}}+\frac{r_{0}}{s_{p}}\right)\right]_{x_{k+1}=0}, \quad k=1, \ldots, K-1 ;
\end{gathered}
$$

- условия равенства температур на спаях $n$ - и $p$-ветвей:

$$
T_{p}^{(k)}(0, t)=T_{n}^{(k)}(0, t), \quad T_{p}^{(k)}(L, t)=T_{n}^{(k)}(L, t), \quad k=1, \ldots, K,
$$

- условия равенства температур между каскадами:

$$
T_{n}^{(k+1)}(0, t)=T_{n}^{(k)}(L, t), \quad k=1, \ldots, K-1,
$$


- условие стабилизации температуры основания охладителя:

$$
T_{n}^{(N)}(L, t)=T_{a}
$$

В этих уравнениях характеристики полупроводниковых материалов ветвей, а именно: коэффициент Зеебека $\alpha(T)$, удельное сопротивление $\rho(T)$, теплопроводность к $(T)$ являются функциями температуры. Экспериментальные температурные зависимости этих характеристик аппроксимируются полиномами. Удельная теплоемкость $c_{n, p}$, площадь сечения ветвей $s_{n, p}$, их длина $L$, количество термоэлементов в каскадах $n_{k}$, электрическое контактное сопротивление $r_{0}$, коэффициент теплообмена $K_{H}$ холодной поверхности устройства с окружающей средой, теплоемкости охлаждаемого объекта и электроизоляционных пластин $g_{k}$, мощность тепловой нагрузки $q_{0}$, температура окружающей среды $T_{a}$ являются константами. Током $I$, который протекает в ветвях и обеспечивает охлаждение, можно управлять, меняя во времени его величину в диапазоне $I \in G_{I}, \quad G_{I}=\left\{0, \quad I_{\max }\right\}$. Одна из рациональных оптимизационных задач заключается в определении оптимальной функции тока $I(t)$, которая минимизирует температуру охлаждения $T_{n}^{(1)}(0, \theta)$ каскадного устройства за определенный промежуток времени $\theta$.

Анализ публикаций $[18,24,25]$ позволяет утверждать, что до настоящего времени для общего случая зависимых от температуры характеристик материалов термоэлемента $\alpha_{n, p}(T), \rho_{n, p}(T), \kappa_{n, p}(T)$ способов решения данной задачи для каскадного охладителя не предложено.

\section{СПОСОБ РЕШЕНИЯ ОПТИМИЗАЦИОННОЙ ЗАДАЧИ}

Эффективным способом решения задачи оптимизации объекта с распределенными параметрами является его дискретизация и переход к объекту с сосредоточенными параметрами, поведение которого описывается системой обыкновенных дифференциальных уравнений.

Переход в уравнениях (1), (3) к безразмерной координате $x=\frac{x_{k}}{L}$, $x \in(0,1)$ и их дискретизация по $x$ позволяют записать уравнения (1), (3) в виде системы обыкновенных дифференциальных уравнений следующим образом.

Обозначим неизвестные температуры $T_{n, p}^{k}(x)$ через $T_{k, m, i}$, где индексы означают: $k=1, \ldots, K$ - номер каскада; $m=1,2$ - номер ветви ( $m=1$ $n$-ветвь, $m=2$ - $p$-ветвь); $i=1, \ldots, N+1$ - номер узла по координате $(N=1 / h, h$ - шаг по координате). Тогда дискретизированные уравнения (1), (3), (4) запишутся в виде системы 


$$
\begin{gathered}
\frac{d T_{k, 1,1}}{\partial t}=F_{k}, \quad k=1, \ldots, K, \\
\frac{d T_{k, m, i}}{\partial t}=f_{k, m, i}, \quad k=1, \ldots, K, \quad m=1,2, \quad i=1, \ldots, N,
\end{gathered}
$$

с начальными условиями

$$
T_{k, m, i}(0)=T_{a}, \quad k=1, \ldots, K, \quad m=1,2, \quad i=1, \ldots, N+1,
$$

и дополнительными условиями на границах (5)-(7), которые принимают вид

$$
\begin{gathered}
T_{k, 2,1}(t)=T_{k, 1,1}(t), \quad T_{k, 2, N+1}(t)=T_{k, 1, N+1}(t), \quad k=1, \ldots, K, \\
T_{k, 1, N+1}(t)=T_{k+1,1,1}(t), \quad k=1, \ldots, K-1, \\
T_{K, 1, N+1}(t)=T_{a} .
\end{gathered}
$$

$F_{k}$ и $f_{k, m i}$ - дискретизированные правые части уравнений (1), (3), (4), которые принимают вид

$$
\begin{gathered}
F_{1}=\frac{n_{1}}{g_{1}}\left[q_{0}-K_{H}\left(s_{n}+s_{p}\right)\left(T_{1,1,1}-T_{a}\right)+\right. \\
+\kappa_{n}\left(T_{1,1,1}\right) \frac{s_{n}}{L} \frac{T_{1,1,2}-T_{1,1,1}}{h}-\alpha_{n}\left(T_{1,1,1}\right) I(t) T_{1,1,1}+ \\
\left.+\kappa_{p}\left(T_{1,2,1}\right) \frac{s_{p}}{L} \frac{T_{1,2,2}-T_{1,2,1}}{h}-\alpha_{p}\left(T_{1,2,1}\right) I(t) T_{1,2,1}+I^{2}(t) r_{0}\left(\frac{1}{s_{n}}+\frac{1}{s_{p}}\right)\right], \\
+\alpha_{n}\left(T_{k-1,1, N+1}\right) I(t) T_{k-1,1, N+1}-\kappa_{p}\left(T_{k-1,2, N+1}\right) \frac{s_{p}}{L} \frac{T_{k-1,2, N+1}-T_{k-1,2, N}}{h}+ \\
\left.+\alpha_{p}\left(T_{k-1,2, N+1}\right) I(t) T_{k-1,2, N+1}+I^{2}(t) r_{0}\left(\frac{1}{s_{n}}+\frac{1}{s_{p}}\right)\right]- \\
-\frac{F_{k}}{g_{k}}\left[-\kappa_{n-1}\left[-\kappa_{n}\left(T_{k-1,1, N+1}\right) \frac{s_{n}}{L} \frac{T_{k-1,1, N+1}-T_{k-1,1, N}}{h}+\right.\right. \\
\left.-\kappa_{p}\left(T_{k, 2,1}\right) \frac{s_{p}}{L} \frac{T_{k, 2,2}-T_{k, 2,1}}{h}+\alpha_{p}\left(T_{k, 2,1}\right) I(t) T_{k, 2,1}-I^{2}(t) r_{0}\left(\frac{1}{s_{n}}+\frac{1}{s_{p}}\right)\right] \\
k=2, \ldots, K, \\
h
\end{gathered}
$$




$$
\begin{gathered}
f_{k, m, i}=\frac{1}{c_{m} L^{2} h^{2}}\left[\kappa_{m}\left(T_{k, m, i+1}\right) T_{k, m, i+1}-\left(\kappa_{m}\left(T_{k, m, i+1}\right)+\kappa_{m}\left(T_{k, m, i}\right)\right) T_{k, m, i}+\right. \\
\left.+\kappa_{m}\left(T_{k, m, i}\right) T_{k, m, i-1}\right]+\rho_{m}\left(T_{k, m, i}\right) \frac{I^{2}(t)}{c_{m} s_{m}^{2}}-\frac{I(t) T_{k, m, i}}{c_{m} s_{m} L} \frac{\partial \alpha_{m}\left(T_{k, m, i}\right)}{\partial T} \frac{T_{k, m, i}-T_{k, m, i-1}}{h} \\
k=1, \ldots, K, \quad m=1,2, \quad i=2, \ldots, N
\end{gathered}
$$

Задача состоит в определении оптимальной функции управления $I(t)$, которая обеспечивает минимум функционала Ф:

$$
\Phi=T_{1,1,1}(\theta) .
$$

Такая оптимизационная задача относится к задачам со свободным правым концом и фиксированным временем.

Решение такой оптимизационной задачи определяется принципом максимума Понтрягина [19]. Для каждого момента времени $t$ должно удовлетворяться условие максимума

$$
H(T(t), I(t), \psi(t))=\max _{I \in G_{I}} H(T(t), I, \psi(t)),
$$

где функция Гамильтона $H$ имеет вид

$$
H=\sum_{k=1}^{K}\left(\Psi_{k} F_{k}+\sum_{m=1}^{2} \sum_{i=2}^{N} \psi_{k, m, i} f_{k, m, i}\right) .
$$

Вспомогательные (сопряженные $T$ ) переменные $\Psi_{k}, \psi_{k, m, i}$ должны удовлетворять системе дифференциальных уравнений

$$
\begin{gathered}
\frac{d \Psi_{1}}{d t}=-\Psi_{1}\left(\frac{\partial F_{1}}{\partial T_{1,1,1}}+\frac{\partial F_{1}}{\partial T_{1,2,1}}\right)-\psi_{1,1,2} \frac{\partial f_{1,1,2}}{\partial T_{1,1,1}}-\psi_{1,2,2} \frac{\partial f_{1,2,2}}{\partial T_{1,2,1}} \\
\frac{d \Psi_{k}}{d t}=-\Psi_{k}\left(\frac{\partial F_{k}}{\partial T_{k, 1,1}}+\frac{\partial F_{k}}{\partial T_{k, 2,1}}+\frac{\partial F_{k}}{\partial T_{k-1,1, N+1}}+\frac{\partial F_{k}}{\partial T_{k-1,2, N+1}}\right)-\psi_{k, 1,2} \frac{\partial f_{k, 1,2}}{\partial T_{k, 1,1}}-\quad(17) \\
-\Psi_{k, 2,2} \frac{\partial f_{k, 2,2}}{\partial T_{k, 2,1}}-\psi_{k-1,1, N} \frac{\partial f_{k-1,1, N}}{\partial T_{k-1,1, N+1}}-\psi_{k-1,2, N} \frac{\partial f_{k-1,2, N}}{\partial T_{k-1,2, N+1}}, \quad k=2, \ldots, K, \\
\frac{d \psi_{k, m, 2}}{d t}=-\Psi_{k} \frac{\partial F_{k}}{\partial T_{k, m, 2}}-\psi_{k, m, 2} \frac{\partial f_{k, m, 2}}{\partial T_{k, m, 2}}-\psi_{k, m, 3} \frac{\partial f_{k, m, 3}}{\partial T_{k, m, 2}}, k=1, \ldots, K, m=1,2, \\
\frac{d \psi_{k, m, i}}{d t}=-\psi_{k, m, i-1} \frac{\partial f_{k, m, i-1}}{\partial T_{k, m, i}}-\psi_{k, m, i} \frac{\partial f_{k, m, i}}{\partial T_{k, m, i}}- \\
-\Psi_{k, m, i+1} \frac{\partial f_{k, m, i+1}}{\partial T_{k, m, i}}, k=1, \ldots, K, m=1,2, i=3, \ldots, N-1,
\end{gathered}
$$




$$
\begin{gathered}
\frac{d \psi_{k, m, N}}{d t}=-\psi_{k, m, N-1} \frac{\partial f_{k, m, N-1}}{\partial T_{k, m, N}}-\psi_{k, m, N} \frac{\partial f_{k, m, N}}{\partial T_{k, m, N}}-\Psi_{k+1} \frac{\partial F_{k+1}}{\partial T_{k, m, N}}, \\
k=1, \ldots K-1, m=1,2, \\
\frac{d \psi_{K, m, N}}{d t}=-\psi_{K, m, N-1} \frac{\partial f_{K, m, N-1}}{\partial T_{K, m, N}}-\psi_{K, m, N} \frac{\partial f_{K, m, N}}{\partial T_{K, m, N}}, m=1,2 .
\end{gathered}
$$

с конечными условиями в виде

$$
\begin{gathered}
\Psi_{1}(\theta)=-1, \\
\Psi_{k}(\theta)=0, \quad k=2, \ldots, K, \\
\Psi_{k, m, i}(\theta)=0, \quad k=1, \ldots, K, \quad m=1,2, \quad i=2, \ldots, N .
\end{gathered}
$$

Производные в уравнениях (17) учитывают дополнительные условия (10).

Совокупностью уравнений (8)-(18) определяется решение поставленной оптимизационной задачи нестационарного термоэлектрического охлаждения.

\section{АЛГОРИТМ ДЛЯ РЕШЕНИЯ ЗАДАЧИ}

Нелинейность правых частей основных дифференциальных уравнений (1), (3), (4) и сложность задачи в целом позволяют решить ее только численными методами. Для этого можно воспользоваться следующим алгоритмом метода последовательных приближений.

Задаем начальное приближение функции управления, например, в виде $I^{(0)}(t)=I_{0}$, где $I_{0} \in G_{I}$.

1. С этим приближением решаем основную систему дифференциальных уравнений (8) с начальными условиями (9), учитывая дополнительные условия (10), и находим вектор-функцию состояний объекта $T(t)$. Для численного метода решения этой системы можно использовать метод конечных разностей.

2. Определяем значение функционала Ф (14).

3. Вычисленную вектор-функцию состояния объекта $T(t)$ и начальное приближение $I^{(0)}(t)$ используем для решения вспомогательной системы $(17) \mathrm{c}$ конечными условиями (18), чтобы вычислить вектор-функции $\Psi(t), \psi(t)$. Для численного решения системы (17) можно применить метод конечных разностей.

4. Используем решения основной и вспомогательной систем для вычисления максимальных значений функции Гамильтона $H$ (16) по переменной $I$ для каждого момента времени $t$. Для выбора оптимального значения $I$ из области $G_{I}$ можно воспользоваться, например, методом золотого сечения. Получим новое приближение вектор-функции управления $I^{(1)}(t)$. 
5. С новой функцией управления решаем основную систему уравнений (8)-(10) и определяем новое значение функционала Ф (14). Повторяем итерационный процесс до достижения изменения функционала Ф в пределах заданной точности.

Такой алгоритм можно реализовать компьютерными методами и найти решение поставленной оптимизационной задачи.

\section{РЕЗУЛЬТАТЫ ОПТИМИЗАЦИИ НЕСТАЦИОНАРНОГО ПРОЦЕССА ТЕРМОЭЛЕКТРИЧЕСКОГО ОХЛАЖДЕНИЯ}

Оценки характеристик нестационарного процесса термоэлектрического охлаждения проводились на примерах одно- и двухкаскадного модулей, ветви которых изготовлены из полупроводниковых материалов на основе $B i-T e \quad n$ - и $p$-типа проводимости. Типичные экспериментальные температурные зависимости термоэлектрических параметров $\alpha_{n, p}(T), \rho_{n, p}(T)$, $\kappa_{n, p}(T)$ этих материалов [18] аппроксимировались полиномами и использовались для расчетов. Теплоемкость материалов $n$ - и $p$-типов предполагалась одинаковой и равной $c=1.4$ Дж/см³. Оценки выполнялись для термоэлементов с высотой ветвей $L=0.14$ см, площадью поперечного сечения $s_{n, p}=0.1 \times 0.1 \mathrm{~cm}^{2}, \quad$ величиной контактного сопротивления $r_{0}=5 \cdot 10^{-6} \mathrm{Oм} \cdot \mathrm{cm}^{2}$. Учитывался теплообмен теплопоглощающей поверхности модулей с окружающей средой, температура которой $T_{0}=300 \mathrm{~K}$, коэффициент теплообмена $K_{H}=10^{-3} \mathrm{BT} / \mathrm{cm}^{2} \mathrm{~K}$. Рассматривалась работа модулей в режиме без тепловой нагрузки, т.е. количество тепла, выделяемого охлаждаемым объектом, принималось равным $q_{0}=0$ Вт. Теплоемкость изоляционных пластин в расчете на один термоэлемент равнялась $g_{k} / n_{k}=0.0012$ Дж/К, коэффициент каскадирования для 2-каскадного модуля $n_{k-1} / n_{k}=1 / 2$.

Предложенный алгоритм решения задачи оптимизации нестационарного процесса термоэлектрического охлаждения был реализован компьютерными методами в среде MATLAB.

Сначала задача (8)-(10) решалась для одно- и двухкаскадного охладителей при условии их питания постоянным током $I$. Примеры решения задачи, которые демонстрируют поведение нестационарного процесса термоэлектрического охлаждения, показаны на рис. 2-3. На рис. 2 показано, как изменяется температура охлаждения $T_{c}$ во времени $t$ при различных значениях постоянного тока питания однокаскадного охладителя. Аналогичные данные были получены в работе [26] несколько иным методом. Сравнение результатов, полученных двумя различными способами, показало, что они совпадают с точностью до $1-1.5 \%$.

На рис. 3 приведены зависимости $T_{c}(t)$ для двухкаскадного устройства. Тут также показано поведение межкаскадной температуры (пунктирные линии). 


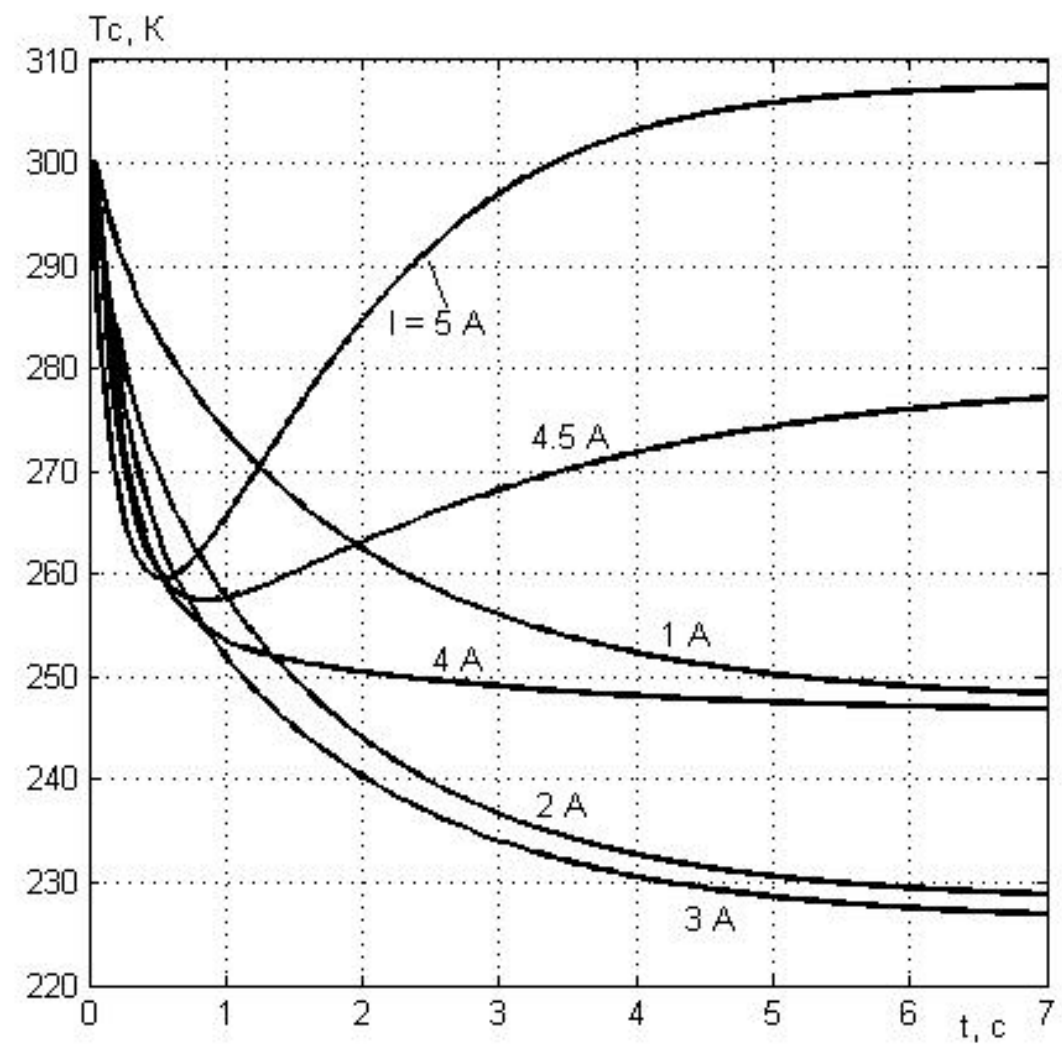

Рис. 2. Зависимости температуры охлаждения $T_{c}$ от времени $t$ для однокаскадного устройства при питании постоянным током $I$.

На рис. 2, 3 видно, что температура охлаждения в начальные моменты времени понижается, а затем зависимости $T_{c}(t)$ достигают насыщения, т.е. значений температуры в стационарном режиме. Очевидно, что существует оптимальное значение тока, при котором достигается самая низкая температура охлаждения. Минимальная температура на однокаскадном охладителе достигается при значении тока $I_{\text {орt }}=3 \mathrm{~A}$ и равна $T_{c}=228 \mathrm{~K}$, на двухкаскадном $T_{c}=207 \mathrm{~K}$ при $I_{\mathrm{opt}}=2.5 \mathrm{~A}$. Время выхода однокаскадного модуля в стационарный режим составляет приблизительно $\theta=5$ с, у двухкаскадного модуля $\theta=8$ c. Таким образом, максимальный перепад температур, который достигается на однокаскадном модуле при $q_{0}=0$ в стационарном режиме, равен $\Delta T_{\max }=72 \mathrm{~K}$, на двухкаскадном $\Delta T_{\max }=93 \mathrm{~K}$. Такие температурные перепады соответствуют уровню охлаждения однокаскадных и двухкаскадных термоэлектрических модулей промышленного производства [27]. 


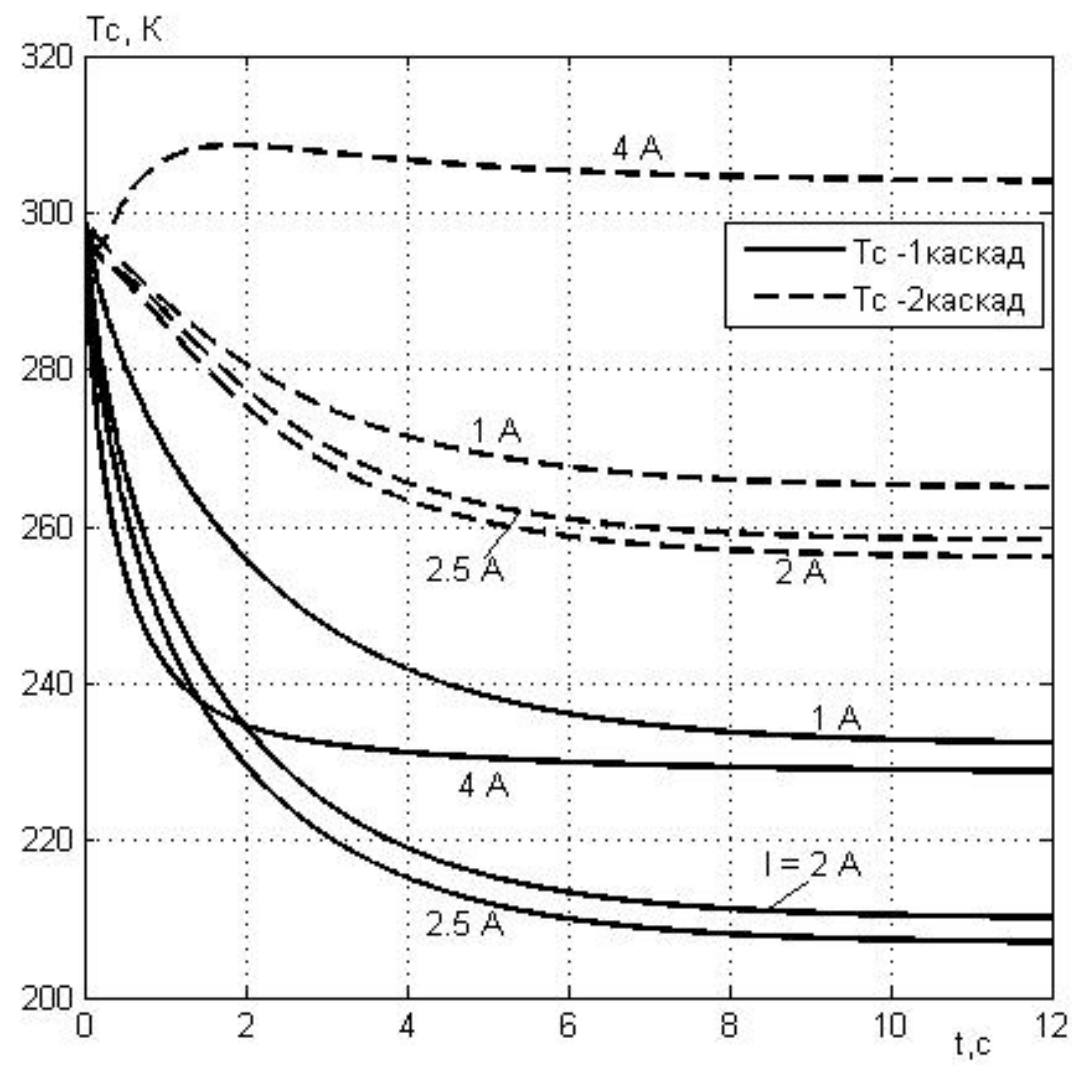

Рис. 3. Зависимости температуры охлаждения $T_{c}$ от времени $t$ для двухкаскадного устройства при питании постоянным током $I$.

Определенные таким образом оптимальные значения тока $I_{\text {opt }}$ использовались в качестве начального приближения для поиска оптимальных функций управления $I(t)$ нестационарными процессами одно- и двухкаскадного термоэлектрического охлаждения.

Примеры результатов расчета оптимальных функций тока питания для одно- и двухкаскадных модулей приведены на рис. 4, 5 соответственно. Тут показаны рассчитанные оптимальные зависимости тока от безразмерного параметра времени $I(t / \theta)$, которые минимизируют температуру охлаждения модулей $T_{c}$ в разные конечные моменты времени $\theta$. На этих же рисунках показано, как понижается температура охлаждения $T_{c}$ в зависимости от времени при использовании таких оптимальных функций тока.

Температура, которая достигается за время $\theta=5$ с на однокаскадном модуле равна $T_{c}=218 \mathrm{~K}$, что на $10 \mathrm{~K}$ ниже минимальной температуры в стационарном режиме. Аналогичные результаты были получены в работе [26] путем использования вариационного метода для поиска оптимальных функций тока, минимизирующих температуру охлаждения однокаскадного устройства. При одинаковых исходных данных для решения задачи результаты совпадают с точностью до $1.5-2 \%$. 
На двухкаскадном модуле достигается $T_{c}=197 \mathrm{~K}$ за $\theta=12$ с, что на $10 \mathrm{~K}$ ниже минимальной температуры в стационарном режиме.

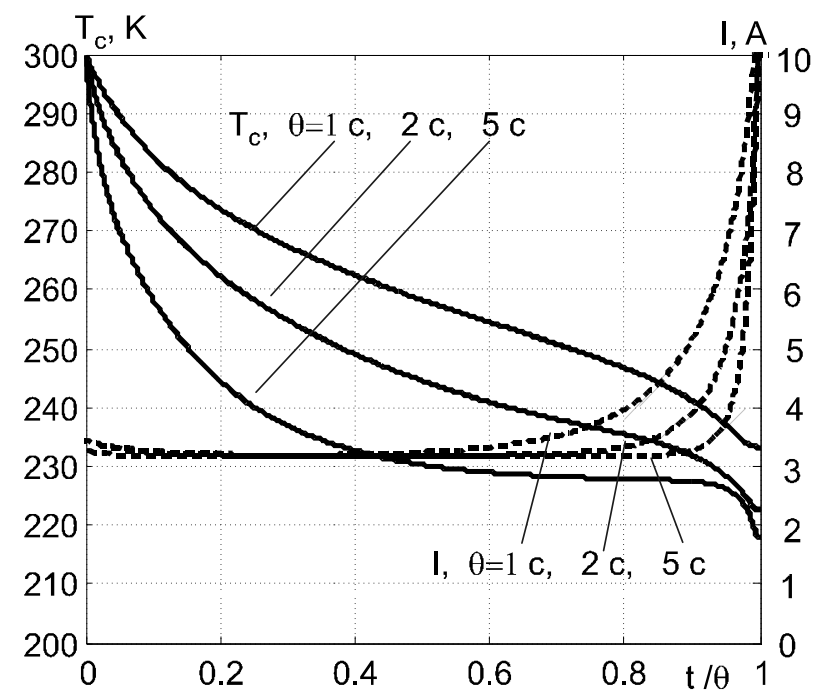

Рис. 4. Оптимальные зависимости тока $I$ и соответствующие зависимости температуры охлаждения $T_{c}$ однокаскадного модуля от безразмерного параметра времени $t / \theta$ для различных интервалов времени $\theta$.

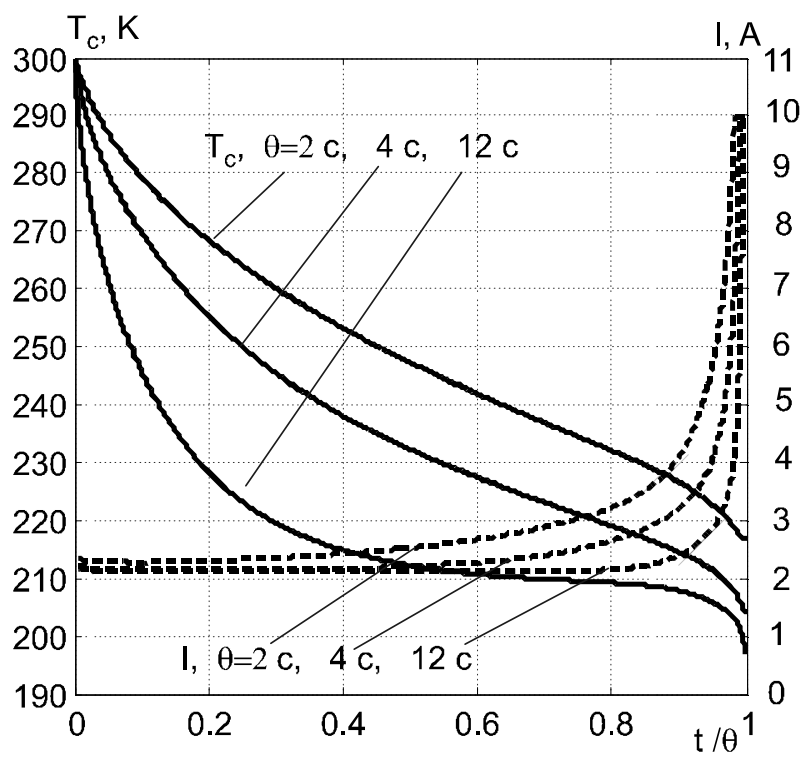

Рис. 5. Оптимальные зависимости тока $I$ и соответствующие зависимости температуры охлаждения $T_{c}$ двухкаскадного модуля от безразмерного параметра времени $t / \theta$ для различных интервалов времени $\theta$.

На рис. 6 показано, какую минимальную температуру можно достичь за различные промежутки времени при использовании оптимальных функций тока питания одно- и двухкаскадного модулей. Эти же данные демонстрируют, за какое минимальное время достигается заданная температура охлаждения. 


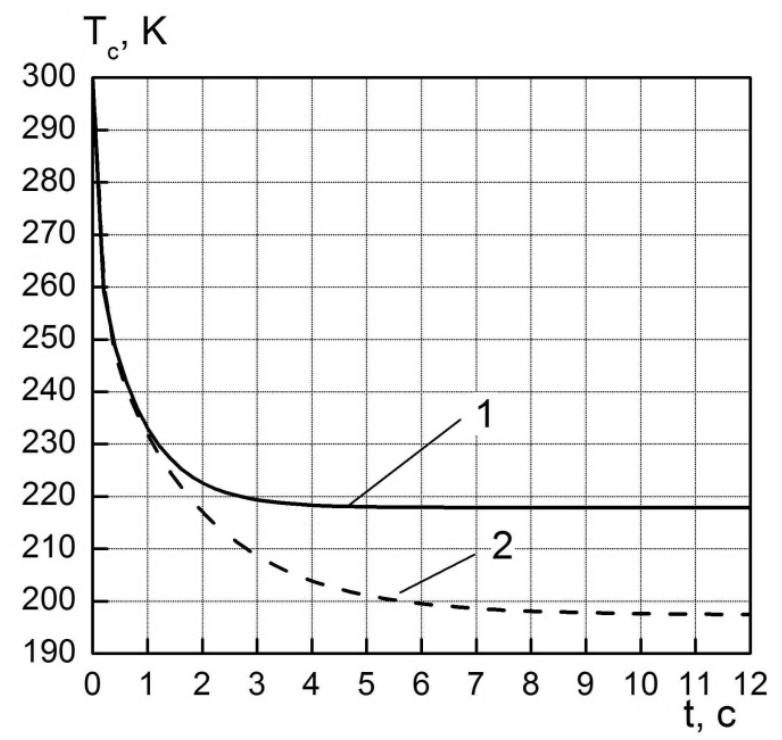

Рис. 6. Минимальная температура $T_{c}$ нестационарного охлаждения, которая достигается за разные промежутки времени $t$ однокаскадным (1) и двухкаскадным (2) охладителями при использовании оптимальных функций тока питания.

Таким образом, полученные результаты оптимизации нестационарного процесса термоэлектрического охлаждения доказывают, что применение оптимальных функций тока позволяет достичь существенно более низких температур, чем это возможно при использовании постоянного тока.

\section{Выводы}

Дискретизация по координате объектов с распределенными параметрами, поведение которых описывается нелинейной системой дифференциальных уравнений параболического типа, дает возможность применить принцип максимума Понтрягина для моделирования оптимальных функций управления и является одним из рациональных способов решения задач оптимального управления такими объектами.

Примером такой задачи является оптимизация процесса нестационарного термоэлектрического охлаждения, результаты решения которой показали, что рассчитанные оптимальные зависимости тока питания от времени для одно- и двухкаскадного термоэлектрических модулей обеспечивают в нестационарном режиме температуру охлаждения на 10 К ниже минимальной температуры в стационарном режиме. Это свидетельствует о перспективности использования нестационарных режимов термоэлектрических устройств, рациональное функционирование которых возможно лишь при решении задач оптимального управления такими режимами.

1. Ahmed N.U. Distributed Parameter Systems / N.U. Ahmed // Encyclopedia of Physical Science and Technology (Third Edition). Academic PRESS, 2003. - P. 561-587. 
2. Alessandri S.A. Feedback Optimal Control of Distributed Parameter Systems by Using Finite-Dimensional Approximation Schemes / S.A. Alessandri, M. Gaggero, R. Zoppoli // IEEE Transactions on Neural Networks and Learning Systems. - 2012. - Vol. 23, No. 6. - P. 984-995.

3. Guangcao Ji. Optimal Boundary Control of the Heat Equation with Target Function at Terminal Time / Ji Guangcao, Martin Clyde // Applied Mathematics and Computation. 2002. - Vol. 127. - P. 335-345.

4. Bokhari M.A. Optimal Control of Parabolic Systems with Infinite Time Horizons / M.A. Bokhari, I.S. Sadek // Applied Mathematics and Computation. - 2008. Vol. 206. - P. 678-684.

5. El-Farra N.H. Analysis and Control of Parabolic PDE Systems with Input Constraints / N.H. El-Farra, A. Armaou, P.D. Christofides // Automatica. - 2003. - Vol. 39. P. 715-725.

6. Kucuka I. Optimal Control of a Distributed Parameter System with Applications to Beam Vibrations Using Piezoelectric Actuators / I. Kucuka, I. Sadeka, Y. Yilmazc // Journal of the Franklin Institute. - 2014. - Vol. 351, issue 2. - P. 656-666.

7. Zuyev A. Partial Asymptotic Stabilization of Nonlinear Distributed Parameter Systems / A. Zuyev // Automatica. - 2005. - Vol. 41. - P. 1-10.

8. Zuazua E. Propagation, Observation, and Control of Waves Approximated by Finite Difference Methods / E. Zuazua / SIAM Rev. — 2005. — Vol. 47, No. 2. — P. 197-243.

9. Krstic M. Boundary Controllers and Observers for the Linearized Schrödinger Equation / M. Krstic, B.-Z. Guo, A. Smyshlyaev // SIAM J. Control Opt. — 2011. — Vol. 49, No. 4. P. 1479-1497.

10. Subas M. An Otimal Control Problem Governed by the Potential of a Linear Schrodinger Equation / M. Subas // Applied Mathematics and Computation. - 2002. - Vol. 131. P. 95-106.

11. Alvarez-Vazquez L.J. Optimal Control of a Bioreactor / L.J. Alvarez-Vazquez, F.J. Fernandez // Applied Mathematics and Computation. - 2010. — Vol. 216. P. 559-575.

12. Ryu Sang-Uk. Optimal Control for an Adsorbate-Induced Phase Transition Model/ Sang-Uk Ryu, Atsushi Yagi // Applied Mathematics and Computation. - 2005. Vol. 171. - P. 420-432.

13. Hoffman K.H. Optimal Control of a Phase Field Model for Solidification / K.H. Hoffman, L. Jiang // Numer. Funct. Anal. and Optimiz. - 1992. - Vol. 13, No. 1\&2. - P. 11-27.

14. Muresan V. Cascade Control of a Residual Water Blunting System / V. Muresan, M. Abrudean, M. Unguresan, T. Colosi // Advances in Electrical and Computer Engineering. - 2014. - Vol. 14, No. 2. - P. 135-144.

15. Егоров А.И. Оптимальное управление тепловыми и диффузными процессами / А.И. Егоров. - М.: Наука, 1978. - 463 с.

16. Geering Hans P. Optimal Control with Engineering Applications / Hans P. Geering. NewYork, Berlin, Heidelberg: Springer-Verlag, 2007. — 134 p.

17. Sethi Suresh P. Optimal Control Theory. Applications to Management Science and Economics / Suresh P. Sethi, Gerald L. Thompson. - Springer Science+Business Media, Inc., 2000. $-505 \mathrm{p}$.

18. Анатычук Л.И. Оптимальное управление свойствами термоэлектрических материалов и приборов / Л.И. Анатычук, В. А. Семенюк. - Черновцы: Прут, 1992. - 263 с.

19. Математическая теория оптимального управления / Л.С. Понтрягин, В.Г. Болтянский, Р.В. Гамкрелидзе, Е.Ф. Мищенко. — М.: Наука, 1976. - 392 с.

20. Бутковский А.Г. Теория оптимального управления системами с распределенными параметрами / А.Г. Бутковский. - М.: Наука, 1965. - 474 с.

21. Сиразетдинов Т.К. Оптимизация систем с распределенными параметрами / Т.К. Сиразетдинов. - М.: Наука, 1977. - 479 с.

22. Фурсиков А.В. Оптимальное управление распределенными системами. Теория и приложения / А.В. Фурсиков. - Новосибирск: Научная книга, 1999. - 352 с. 
23. Шевяков А.А. Управление тепловыми объектами с распределенными параметрами / А.А. Шевяков, Р.В. Яковлєва. - М.: Энергоатомиздат, 1986. - 208 с.

24. Анатычук Л.И. Термоэлектричество. Том II. Термоэлектрические преобразователи энергии / Л.И. Анатычук. - Киев, Черновцы: Институт термоэлектричества, 2003. $376 \mathrm{c}$.

25. Yang Ronggui. Transient cooling of thermoelectric coolers and its applications for microdevices / Ronggui Yang, Gang Chen, A. Ravi Kumar, G. Jeffrey Snyder, Jean-Pierre Fleurial // Energy Conversion and Management. - 2005. — Vol. 46. - P.1407-1421.

26. Коцур М.П. Наближений метод оптимального керування в задачах нестаціонарного термоелектричного охолодження / М.П. Коцур // Журнал обчислювальної та прикладної математики. — 2013. — № 4 (114). — С. 37-47.

27. Однокаскадные термоэлектрические модули. Available at: http://www.kryothermtec.com/ru/standsard-single-stage-thermoelectric-coolers.html. Двухкаскадные термоэлектрические модули. Available at: http://www.kryothermtec.com/ru/2-stage-thermoelectric-coolers.html.

UDC 517.977

\title{
OPTIMAL CONTROL BY TRANSIENT MODE OF STAGE THERMOELECTRIC COOLER
}

\author{
M.P. Kotsur ${ }^{1}$, A.G. Nakonechniy ${ }^{2}$ \\ ${ }^{1}$ Yuriy Fedkovych Chernivtsi National University \\ ${ }^{2}$ Taras Shevchenko National University of Kyiv
}

Introduction. A large variety of optimization problems is related to distributed parameter systems (DPSs), whose behavior is described by partial differential equations. The optimal control theory is widely employed for optimization of lumped parameter systems (LPSs), which are modeled by ordinary differential equations. Development of the theory and methods for obtaining optimal control functions for DPSs is much more difficult as compared with solving such problem for LPSs.

The purpose of the present paper is to obtain optimality conditions and to develop numerical methods for solving the optimization problem of an unsteady one-dimensional process with distributed parameters, as well as their application to optimization of transient thermoelectric cooling.

Methods. A method is proposed for solving of optimal control problem for DPS described by nonlinear partial differential equations of parabolic type with nonstandard boundary conditions. A method consists in coordinate discretization of distributed object and transition to the problem for LPS. Optimal control theory based on the Pontryagin maximum principle can be used for such system optimization.

Results. This method is applied for optimization of transient thermoelectric cooling process. Optimal dependences of current on time have been calculated for stage thermoelectric cooler power supply with the purpose of minimizing the cooling temperature within a preset time interval. Results of computer experiment for one- and two-stage coolers are presented.

Conclusion. The obtained results show that the implementation of optimal functions for cooler power supply can provide the cooling temperature in transient mode by $10 \mathrm{~K}$ lower than minimum temperature reached at direct current. 
Keywords: distributed parameter system, optimal control, Pontryagin maximum principle, optimization, transient thermoelectric cooling.

1. Ahmed N.U. Distributed Parameter Systems. Encyclopedia of Physical Science and Technology (Third Edition), Academic PRESS, 2003, pp. 561-587.

2. Alessandri S.A., Gaggero M., Zoppoli R. Feedback Optimal Control of Distributed Parameter Systems by Using Finite-Dimensional Approximation Schemes. IEEE Transactions on Neural Networks and Learning Systems, 2012, vol. 23, no. 6, pp. 984-995.

3. Guangcao Ji, Clyde Martin. Optimal Boundary Control of the Heat Equation with Target Function at Terminal Time. Applied Mathematics and Computation, 2002, vol. 127, pp. 335-345.

4. Bokhari M.A., Sadek I.S. Optimal Control of Parabolic Systems with Infinite Time Horizons. Applied Mathematics and Computation, 2008, vol. 206, pp. 678-684.

5. El-Farra N.H., Armaou A., Christofides P.D. Analysis and Control of Parabolic PDE Systems with Input Constraints. Automatica, 2003, vol. 39, pp. 715-725.

6. Kucuka I., Sadeka I., Yilmazc Y. Optimal Control of a Distributed Parameter System with Applications to Beam Vibrations Using Piezoelectric Actuators. Journal of the Franklin Institute, 2014, vol. 351, issue 2, February, pp. 656-666.

7. Zuyev A. Partial Asymptotic Stabilization of Nonlinear Distributed Parameter Systems. Automatica, 2005, vol. 41, pp. 1-10.

8. Zuazua E. Propagation, Observation and Control of Waves Approximated by Finite Difference Methods. SIAM Rev., 2005, vol. 47, no. 2, pp. 197-243.

9. Krstic M., Guo B.-Z., Smyshlyaev A. Boundary Controllers and Observers for the Linearized Schrödinger Equation. SIAM J. Control Opt., 2011, vol. 49, no. 4, pp. 1479-1497.

10. Subas M. An Otimal Control Problem Governed by the Potential of a Linear Schrodinger Equation. Applied Mathematics and Computation, 2002, vol. 131, pp. 95-106.

11. Alvarez-Vazquez L.J., Fernandez F.J. Optimal Control of a Bioreactor. Applied Mathematics and Computation, 2010, vol. 216, pp. 559-575.

12. Ryu Sang-Uk, Yagi Atsushi. Optimal Control for an Adsorbate-Induced Phase Transition Model. Applied Mathematics and Computation, 2005, vol. 171, pp. 420-432.

13. Hoffman K.H., Jiang L. Optimal Control of a Phase Field Model for Solidification. Numer. Funct. Anal. and Optimiz., 1992, vol. 13, no. 1\&2, pp. 11-27.

14. Muresan V., Abrudean M., Unguresan M., Colosi T. Cascade Control of a Residual Water Blunting System. Advances in Electrical and Computer Engineering, 2014, vol. 14, no. 2, pp. $135-144$.

15. Egorov A.I. Optimal control by thermal and diffuse processes. Moscow: Science, 1978, 463 p. (in Russian).

16. Geering Hans P. Optimal Control with Engineering Applications. NewYork, Berlin, Heidelberg: Springer-Verlag, 2007. $134 \mathrm{p}$.

17. Sethi Suresh P. Optimal Control Theory. Applications to Management Science and Economics. Springer Science+Business Media, Inc., 2000. 505 p.

18. Anatychuk L.I. Optimal Control by Properties of Thermoelectric Materials and Devices. Chernivtsi: Prut, 1992. 263 p. (in Russian).

19. Pontryagin L.S., Boltyanski V.G., Gamkrelidze R.S., Mischenko E.F. The Mathematical Theory of Optimal Processes. Moscow: Nauka, 1976. 392 p. (in Russian).

20. Butkovskii A.G. Theory of Optimal Control by Distributed Parameters Systems. Moscow: Nauka, 1965. 474 p. (in Russian).

21. Sirazetdinov T.K. Optimization of Distributed Parameters Systems. Moscow: Nauka, 1977. 479 p. (in Russian).

22. Fursikov A.V. Optimal Control by Distributed Systems. Theory and Application. Novosibirsk: Nauchnaya Kniga, 1999. 352 p. (in Russian).

23. Shevyakov A.A., Yakovleva R.V. Control by Thermal Objects with Distributed Parameters. Moscow: Energoatomizdat, 1986. 208 p. (in Russian). 
24. Anatychuk L.I. Thermoelectricity. Vol. II. Thermoelectric Energy Convectors. Kiev, Chernovtsi: Institute of Thermoelectricity, 2003.376 p.

25. Yang Ronggui, Chen Gang, Kumar A. Ravi, Snyder G. Jeffrey, Fleurial Jean-Pierre. Transient Cooling of Thermoelectric Coolers and its Applications for Microdevices. Energy Conversion and Management, 2005, vol. 46, pp.1407-1421.

26. Kotsur M.P. Approximate Method of Optimal Control in Problems of Transient Thermoelectric Cooling. Journal of Computational and Applied Mathematics, 2013, vol. 114 , no. 4, pp. 37-47. (in Ukrainian)

27. One-stage thermoelectric modules. Available at: http://www.kryothermtec.com/ru/standsardsingle-stage-thermoelectric-coolers.html.

Two-stage thermoelectric modules. Available at: http://www.kryothermtec.com/ru/2-stagethermoelectric-coolers.html.

Получено 18.02.2015 


\title{
Медицинская и биологическая кибернетика
}

\author{
УдК 517.925.5 \\ ДИСКРЕТНЫЕ ЭФФЕКТЫ В НЕПРЕРЫВНЫХ \\ МОДЕЛЯХ СУКЦЕССИОННЫХ ПРОЦЕСОВ
}

\author{
С.В. Чернышенко, Р.В. Рузич
}

Хмельницкий национальный университет

\begin{abstract}
Модель разомкнутого гиперцикла Эйгена используется в статье для описания длительных экологических сукцессий. Проанализирован многомерный случай; показано, что во многих случаях исследование поведения $n$-мерной системы может быть сведено к исследованию систем меньшей размерности. Квазидискретная динамика модели объясняется через ее бифуркационные свойства, которые вызывают пошаговое изменение структуры системы.
\end{abstract}

Ключевые слова: сукцессия, дискретный процесс, непрерывная модель, гиперцикл Ейгена, бифуркация.

Модель розімкнутого гіперциклу Ейгена використовується в статті для опису тривалих екологічних сукцесій. Проаналізовано багатовимірний випадок; показано, що в багатьох випадках дослідження поведінки $n$-вимірної системи може бути зведено до дослідження систем меншої вимірності. Квазі-дискретна динаміка моделі пояснюється біфуркаційними властивостями, що викликають покрокову зміну структури системи.

Ключові слова: сукцесія, дискретний процес, неперервна модель, гіперцикл Ейгена, біфуркація.

\section{ВВЕДЕНИЕ}

Экологические системы сложны и разнообразны: они состоят из большого числа элементов и, соответственно, характер их динамики определяется большим количеством связей между этими элементами. В то же время, существуют динамические свойства, общие для большинства реальных экологических систем. Одним из таких свойств является сукцессионный характер динамики [1], т. е. пошаговое изменение основных свойств системы в процессе ее движения к устойчивому состоянию. Нарушение стабильного режима функционирования экосистемы на некоторой территории обычно не может быть ликвидировано путем простого возврата в исходное состояние; это происходит путем дискретной смены нескольких экологических ассоциаций.

Существует два традиционных пути в моделировании сукцессий: дискретное описание на основе матриц перехода $[2,3]$ и непрерывное динамическое описание с использованием моделей конкуренции $[4,5]$.

В настоящей работе рассматриваются модели второго типа. Часто инструментом, с помощью которого можно описать сукцессии, являются дифференциальные модели, например, вольтерровского типа [6]. В этих

(C) С.В. Чернышенко, Р.В. Рузич, 2015 
моделях основной движущей силой сукцессионных процессов считается конкуренция. Заметим, что подобного взгляда на природу сукцессий придерживается достаточно большое количество исследователей $[7,8]$. К недостаткам подобных моделей можно отнести отсутствие явных связей между стадиями сукцессии и изменением абиотических элементов системы. Модель отрытого гиперцикла Эйгена позволяет в некоторой степени учесть такую связь; в этом ее явное преимущество перед вольтерровскими системами. Другим достоинством рассматриваемой модели является явно выраженная дискретность ее поведения при непрерывности всех входящих в нее функций. Заметим, что подобный характер динамики присущ большинству экосистем. Модель позволяет показать, что дискретность реальных систем в некоторых случаях может быть объяснена нелинейными эффектами при взаимодействии их элементов.

Цель настоящей работы состоит в том, чтобы выявить нелинейные свойства системы, ответственные за дискретность ее поведения.

\section{МОДЕЛЬ РАЗОМКНУТОГО ГИПЕРЦИКЛА ЕЙГЕНА}

Одной из непрерывных моделей сукцессий является специальная модификация известного гиперцикла Ейгена, предложенная одним из авторов в роботе [9]:

$$
\dot{x}_{i}=\left(F_{i}(x)-\frac{1}{S_{0}} \sum_{j=1}^{n} x_{j} F_{j}(x)\right) x_{i}, \quad i=\overline{1, n}
$$

где $F_{i}(x)=a_{i-1} x_{i-1}-x_{i}, \quad i=\overline{1, n} ; \quad a_{i}>0, \quad i=\overline{1, n-1} ; \quad x_{0}=1, \quad a_{0}=N, \quad N>0$, $S_{0}>0 ; x_{i}$ - численность (биомасса) ассоциаций; $N-$ коэффициент, который задает значения численности равновесия для первой ассоциации, при отсутствии второй; $a_{i}-$ коэффициент, отражающий зависимость $(i+1)$-ой ассоциации от $i$-ой; $S_{0}$ - емкость среды.

В работе представлены некоторые новые результаты исследования динамических свойств модели (1).

Структура строк матрицы Якоби системы (1) имеет вид:

$$
\underbrace{f_{1} \ldots f_{1}}_{m} \underbrace{f_{2} \ldots f_{2}}_{l} f_{3} \underbrace{f_{4} \ldots f_{4}}_{d},
$$

где $f_{1}=-\frac{x_{i}}{S_{0}}\left(a_{k-1} x_{k-1}-2 x_{k}+a_{k} x_{k+1}\right), f_{2}=a_{i-1} x_{i}-\frac{x_{i}}{S_{0}}\left(a_{i-2} x_{i-2}-2 x_{i-1}+a_{i-1} x_{i}\right)$,

$$
f_{3}=\left\{\begin{array}{l}
a_{i-1} x_{i-1}-2 x_{i}-\frac{1}{S_{0}} \sum_{j=1}^{n}\left(a_{j-1} x_{j-1} x_{j}-x_{j}^{2}\right)-\frac{x_{i}}{S_{0}}\left(a_{i-1} x_{i-1}-2 x_{i}+a_{i} x_{i+1}\right), k<n, \\
a_{i-1} x_{i-1}-2 x_{i}-\frac{1}{S_{0}} \sum_{j=1}^{n}\left(a_{j-1} x_{j-1} x_{j}-x_{j}^{2}\right)-\frac{x_{i}}{S_{0}}\left(a_{i-1} x_{i-1}-2 x_{i}\right), k=n,
\end{array}\right.
$$




$$
f_{4}=\left\{\begin{array}{l}
-\frac{x_{i}}{S_{0}}\left(a_{k-1} x_{k-1}-2 x_{k}+a_{k} x_{k+1}\right), k<n, \\
-\frac{x_{i}}{S_{0}}\left(a_{k-1} x_{k-1}-2 x_{k}\right), k=n,
\end{array}\right.
$$

где $m, l, d$ - количества ячеек в строке матрицы Якоби, значения которых вычисляются следующим образом:

$$
m=(i-2), \quad l=\left\{\begin{array}{l}
1, i>1, \\
0, i=1,
\end{array} \quad d=n-m-l-1, \quad(x)=\left\{\begin{array}{l}
x, x \geq 0 \\
0, x<0
\end{array}\right.\right.
$$

где $k$ - номер столбца матрицы Якоби, $k=\overline{1, n}, i$ - номер строки матрицы Якоби, $i=\overline{1, n}$.

Пусть некоторая координата $x_{p}$ стационарной точки $D$ системы (1) равна нулю. Тогда строку $p$ матрицы Якоби можно записать как

$$
\underbrace{0 \ldots 0}_{p-1} \quad a_{p-1} x_{p-1}-\frac{1}{S_{0}} \sum_{j=1}^{n}\left(a_{j-1} x_{j-1} x_{j}-x_{j}^{2}\right) \underbrace{0 \ldots 0}_{n-p} \text {. }
$$

Характеристическое уравнение матрицы Якоби имеет вид:

$$
|J-\lambda I|=0,
$$

где $J$ - матрица Якоби, $I-$ единичная матрица, $\lambda-$ собственное значение.

Запишем характеристическое уравнение матрицы Якоби в точке $D$

$$
\left(\lambda^{*}-a_{p-1} x_{p-1}+\frac{1}{S_{0}} \sum_{j=1}^{n}\left(a_{j-1} x_{j-1} x_{j}-x_{j}^{2}\right)\right) A_{p}=0
$$

где $A_{p}$ - минор по диагональному элементу, который находится в строке $p$ определителя.

Тогда одно собственное значение матрицы Якоби в этой точке может быть рассчитано как

$$
a_{p-1} x_{p-1}-\frac{1}{S_{0}} \sum_{j=1}^{n}\left(a_{j-1} x_{j-1} x_{j}-x_{j}^{2}\right)
$$

или как

$$
-\frac{1}{S_{0}} \sum_{j=1}^{n}\left(a_{j-1} x_{j-1} x_{j}-x_{j}^{2}\right)
$$

в случае $x_{p-1}=0$. Полученный результат сформулируем в форме теоремы. 
Теорема 1. Если координата $x_{p}$ стационарной точки модели (1) равна нулю, то одно из собственных значений матриџы Якоби в этой точке может быть получено из выражения

$$
a_{p-1} x_{p-1}-\frac{1}{S_{0}} \sum_{j=1}^{n}\left(a_{j-1} x_{j-1} x_{j}-x_{j}^{2}\right)
$$

при условии, что предшествующая ей координата ненулевая или $x_{p}$ первая координата.

В случае предиествующей нулевой координаты это выражение имеет вид:

$$
-\frac{1}{S_{0}} \sum_{j=1}^{n}\left(a_{j-1} x_{j-1} x_{j}-x_{j}^{2}\right) .
$$

Определение 1. Особая точка $n$-мерной модели называется точкой«потомком», если первые ее координаты соответствуют координатам особой точки модели меньшей размерности, а другие координать - нули.

Используя теорему 1 , легко доказать следующее утверждение.

Теорема 2. Пусть особая точка $A$ n-мерной модели разомкнутого гипериикла Эйгена является точкой-«потомком» $(n-k)$-мерной модели. Указанную $(n-k)$-мерную модель назовем базовой. Тогда $n-k$ собственных значений матриць Якоби в точке $A$ определяются из базовой системы, $(k-1)$ - из формуль (3), а одно значение равно

$$
a_{n-k} x_{n-k}-S_{0}^{-1} \sum_{j=1}^{n-k}\left(a_{j-1} x_{j-1} x_{j}-x_{j}^{2}\right) .
$$

\section{ЭВОЛЮЦИЯ ЭКОЛОГИЧЕСКИХ СИСТЕМ}

Рассмотрим особую точку $n$-мерной модели (1) с последними $k(k \geq 2)$ нулевыми координатами. В соответствии с теоремой 2 первые $n-k$ собственных значений совпадают с собственными значениями в точке $(n-k)$-мерного пространства базовой модели. Если соответствующая особая точка $(n-k)$-мерной модели устойчива, то $n-k$ собственных значений матрицы Якоби для $n$-мерной модели имеют отрицательные действительные части. Остальные собственные значения определяются формулами

$$
\lambda_{1}=a_{n-k} x_{n-k}-\frac{1}{S_{0}} \sum_{j=1}^{n-k}\left(a_{j-1} x_{j-1} x_{j}-x_{j}^{2}\right)
$$

и

$$
\lambda_{i}=-\frac{1}{S_{0}} \sum_{j=1}^{n-k}\left(a_{j-1} x_{j-1} x_{j}-x_{j}^{2}\right), i=\overline{1, k-1}
$$


Поскольку рассматривается только неотрицательная область фазового пространства, то разница $\lambda_{1}-\lambda_{i}=a_{n-k} x_{n-k}, i=\overline{1, k-1}$, положительная, что, в свою очередь, подтверждает справедливость неравенства $\lambda_{1} \geq \lambda_{i}$. Таким образом, если собственное значение $\lambda_{1}$ отрицательное, то и другие собственные значения $\lambda_{i}$ также отрицательные. Следовательно, если особая точка с последней координатой ноль $(n-k+1)$-мерной модели устойчива, то устойчива и точка-«потомок» $n$-мерной модели. Причем интервалы для параметров, при которых точка устойчива, не меняются. Полученный результат сформулируем в виде теоремы.

Теорема 3. Если особая точка с последней нулевой координатой $n$-мерной модели разомкнутых гиперииклов Эйгена устойчива, то устойчива u точка-«потомок» модели высшей размерности, причем промежуток параметров, при которых точка устойчива, не меняется. В противном случае точка-«потомок» неустойчива.

Этот результат можно усилить. Отметим, что $n$-мерная система (1) имеет $n+1$ потенциально устойчивое равновесное состояние. Первые $n-1$ точки могут рассматриваться как точки-«потомки» системы размерности $(n-1)$. Bсе эти точки имеют нулевую последнюю координату, т.е. для них справедлива теорема 3. Изменение топологии системы с ростом $S_{0}$ (бифуркации) сводится к последовательному обмену устойчивостью между $n-1$ потенциально устойчивыми точками (при этом переход из $n$-го состояния к $(n-1)$-му состоянию, которое соответствует избытку ресурсов в экологической системе, не является бифуркацией).

Справедливо утверждение.

Теорема 4. Первые $n-2$ бифуркационные условия для $n$-мерной модели разомкнутого гиперцикла Эйгена совпадают $c$ первыми $n-2$ бифуркационными условиями для модели размерности $n-1$.

Для полного описания эволюции системы необходимо показать, что особая точка с одной (и последней) нулевой координатой $(n+1)$-мерной системы, которая является потомком устойчивой особой точки $n$-мерной системы, устойчива. Пока не удалось доказать справедливость данного утверждения в общем случае. Оно верно для трехмерного случая [10]; покажем его справедливость для четырехмерного случая. Рассмотрим точку

$$
\left(\frac{S_{0}+N\left(a_{2}+2\right)}{a_{1} a_{2}+a_{1}+a_{2}+3}, \frac{\left(a_{1}+1\right) S_{0}+N\left(a_{1}-1\right)}{a_{1} a_{2}+a_{1}+a_{2}+3}, \frac{\left(a_{1} a_{2}+a_{2}+1\right) S_{0}-N\left(a_{1}+a_{2}+1\right)}{a_{1} a_{2}+a_{1}+a_{2}+3}, 0\right) .
$$

Согласно теореме 2, три собственных значения матрицы Якоби в этой точке являются отрицательными при $\frac{S_{0}}{N} \in\left(\frac{a_{1}+a_{2}+1}{a_{2}+a_{1} a_{2}+1}, a_{1}+a_{1} a_{2}+1\right), \quad$ а четвертое значение 


$$
\lambda_{4}=\frac{1+a_{3}+a_{2} a_{3}+a_{1} a_{2} a_{3}}{3+a_{1}+a_{2}+a_{1} a_{2}} S_{0}-\frac{1+a_{1}+a_{3}+a_{1} a_{2}+a_{1} a_{3}+a_{2} a_{3}}{3+a_{1}+a_{2}+a_{1} a_{2}} N .
$$

Таким образом, указанная точка устойчива, если

$$
\frac{S_{0}}{N} \in\left(\frac{a_{1}+a_{2}+1}{a_{2}+a_{1} a_{2}+1}, \frac{1+a_{1}+a_{3}+a_{1} a_{2}+a_{1} a_{3}+a_{2} a_{3}}{1+a_{3}+a_{2} a_{3}+a_{1} a_{2} a_{3}}\right) .
$$

Другое важное утверждение: когда размер экологической ниши (параметр $S_{0}$ ) превышает некоторое критическое значение, точка $\left(N, a_{1} N, a_{1} a_{2} N, \ldots, N \prod_{j=1}^{n-1} a_{j}\right)$ становится устойчивой. Докажем справедливость этого утверждения для четырехмерного случая.

Характеристическое уравнение матрицы Якоби в точке $\left(N, a_{1} N, a_{1} a_{2} N, a_{1} a_{2} a_{3} N\right)$ имеет вид

$$
\begin{gathered}
\lambda^{4}+\left(S_{0}\left(1+a_{1}+a_{1} a_{2}+a_{1} a_{2} a_{3}\right)-N\right) S_{0}^{-1} N \lambda^{3}+ \\
+a_{1}\left(S_{0}\left(a_{1} a_{2}+a_{1} a_{2} a_{3}+a_{2} a_{3}+a_{1} a_{2}^{2}+a_{2}+1\right)-\left(a_{2}+a_{1}+a_{2} a_{3}+1\right) N\right) S_{0}^{-1} N^{3} \lambda^{2}+ \\
+a_{1} a_{2}\left(a_{1}^{2} a_{2} a_{3}+a_{1} a_{2} a_{3}+a_{1} a_{3}+a_{1}\right) N^{3} \lambda- \\
-a_{1} a_{2}\left(a_{1}^{2} a_{2}+a_{1} a_{2} a_{3}+a_{1}+a_{1} a_{3}+a_{1}^{2}+a_{1}^{2} a_{3}\right) S_{0}^{-1} N^{4} \lambda+ \\
+N^{4} a_{1}^{3} a_{2}^{2} a_{3}\left(S_{0}-\left(a_{1} a_{2} a_{3}+a_{1} a_{2}+a_{1}+1\right) N\right)=0 .
\end{gathered}
$$

Используя теорему Ньютона [11], легко показать, что точка $\left(N, a_{1} N, a_{1} a_{2} N, a_{1} a_{2} a_{3} N\right)$ устойчива при $S_{0}>\left(1+a_{1}+a_{1} a_{2}+a_{1} a_{2} a_{3}\right) N$.

Важно заметить, что существует интервал

$$
\frac{S_{0}}{N} \in\left(\frac{1+a_{1}+a_{3}+a_{1} a_{2}+a_{1} a_{3}+a_{2} a_{3}}{1+a_{3}+a_{2} a_{3}+a_{1} a_{2} a_{3}}, 1+a_{1}+a_{1} a_{2}+a_{1} a_{2} a_{3}\right)
$$

на котором, как минимум, еще одна стационарная точка является устойчивой. Предположим, что такой точкой является

$$
\begin{gathered}
M=\left(\left(S_{0}+N\left(a_{2}+a_{3}+a_{2} a_{3}+3\right)\right) K,\left(S_{0}\left(a_{1}+1\right)+N\left(2 a_{1}+a_{1} a_{3}-1\right)\right) K,\right. \\
S_{0}\left(1+a_{2}+a_{1} a_{2}\right) K+N\left(a_{2} a_{1}-a_{1}-a_{2}-1\right) K, \\
\left.\left(S_{0}\left(a_{3}+a_{2} a_{3}+a_{1} a_{2} a_{3}+1\right)-N\left(a_{1}+a_{3}+a_{1} a_{2}+a_{1} a_{3}+a_{2} a_{3}+1\right)\right) K\right),
\end{gathered}
$$

где $K=\left(a_{1}+a_{2}+a_{3}+a_{1} a_{2}+a_{2} a_{3}+a_{1} a_{2} a_{3}+4\right)^{-1}$.

Запишем характеристическое уравнение матрицы Якоби в точке $M$ :

$$
\left(\lambda-\frac{S_{0}-\left(1+a_{1}+a_{1} a_{2}+a_{1} a_{2} a_{3}\right) N}{a_{1}+a_{2}+a_{3}+a_{1} a_{2}+a_{2} a_{3}+a_{1} a_{2} a_{3}+4}\right)\left(\lambda^{3}+c_{1} \lambda^{2}+c_{2} \lambda+c_{3}\right)=0,
$$

где 


$$
\begin{aligned}
c_{1}= & \left.S_{0}+N\right)\left(S_{0}\left(a_{1}+a_{2}+a_{3}+a_{1} a_{2}+a_{2} a_{3}+a_{1} a_{2} a_{3}+3\right)-N\left(a_{2}+a_{3}+a_{2} a_{3}+3\right)\right) \\
c_{2}= & S_{0}^{-1}\left(a_{1}+a_{2}+a_{3}+a_{1} a_{2}+a_{2} a_{3}+a_{1} a_{2} a_{3}+4\right)^{-2}\left(S _ { 0 } ^ { 3 } \left(\left(a_{2} a_{3}+a_{2}^{2} a_{3}+a_{2}\right) a_{1}^{2}+\right.\right. \\
& +\left(4 a_{2} a_{3}+a_{3}+2 a_{2}^{2} a_{3}+3 a_{2}+2\right) a_{1}+3 a_{2} a_{3}+a_{2}^{2} a_{3}+ \\
+ & \left.2 a_{2}+2 a_{3}+3\right)+S_{0}^{2} N\left(\left(3 a_{2}+3 a_{2}^{2} a_{3}+3 a_{2} a_{3}-a_{3}+a_{2} a_{3}^{2}+a_{2}^{2} a_{3}^{2}-2\right) a_{1}^{2}+\right. \\
& +\left(7 a_{2} a_{3}+a_{2}^{2}+a_{3}^{2}+3 a_{2} a_{3}^{2}+5 a_{2}+2+2 a_{2}^{2} a_{3}^{2}+3 a_{2}^{2} a_{3}+2 a_{3}\right) a_{1}+ \\
& \left.+a_{2}^{2}+a_{3}^{2}+a_{2}^{2} a_{3}^{2}+a_{2}+a_{2} a_{3}+a_{3}+2 a_{2} a_{3}^{2}\right)+s_{3}^{2}-5 a_{3}+\left(\left(2 a_{2}^{2} a_{3}+7\right) a_{1}^{2}-\right. \\
- & \left(5 a_{2}+3 a_{3}+3 a_{2}^{2} a_{3}+a_{3}^{2}+7 a_{2} a_{3}+a_{2}^{2} a_{3}^{2}+2 a_{2} a_{3}^{2}+4\right) a_{1}-9-11 a_{2} a_{3}- \\
-2 & \left.a_{2}^{2} a_{3}^{2}-8 a_{3}-2 a_{3}^{2}-8 a_{2}-2 a_{2}^{2}-3 a_{2}^{2} a_{3}-4 a_{2} a_{3}^{2}\right)+N^{3}\left(-a_{1}^{2}\left(a_{3}^{2}+2 a_{2}+5 a_{3}+\right.\right. \\
& \left.+3 a_{2} a_{3}+a_{2} a_{3}^{2}+6\right)-a_{1}\left(a_{2} a_{3}^{2}+4 a_{2} a_{3}+a_{2}^{2}+3 a_{2}+a_{2}^{2} a_{3}^{2}+2 a_{2}^{2} a_{3}\right)+ \\
& \left.\left.+2 a_{2}^{2} a_{3}+7 a_{2} a_{3}+6+5 a_{2}+a_{2}^{2}+a_{3}^{2}+5 a_{3}+a_{2}^{2} a_{3}^{2}+2 a_{2} a_{3}^{2}\right)\right) \\
c_{3}=N^{4} & S_{0}^{-1}\left(a_{1}+a_{2}+a_{3}+a_{1} a_{2}+a_{2} a_{3}+a_{1} a_{2} a_{3}+4\right)^{-3}\left(a_{1}+1\right)\left(1+a_{3}+a_{2} a_{3}+a_{1} a_{2} a_{3}\right) \times \\
& \times\left(a_{2}+a_{1} a_{2}+1\right)\left(\frac{S_{0}}{N}+a_{2} a_{3}+a_{3}+a_{2}+2\right)\left(\frac{S_{0}}{N}+\frac{a_{1} a_{2}-a_{1}-a_{2}-1}{1+a_{2}+a_{1} a_{2}}\right) \times \\
& \times\left(\frac{S_{0}}{N}+\frac{a_{1} a_{3}+2 a_{1}-1}{1+a_{1}}\right)\left(\frac{S_{0}}{N}-\frac{1+a_{1} a_{3}}{1}\right) \\
& \left(a_{3}+a_{1} a_{2}+a_{2} a_{3}+a_{1} a_{3}\right)
\end{aligned}
$$

Очевидно, что одно из собственных значений отрицательно, если $\frac{S_{0}}{N} \in\left(0 ; 1+a_{1}+a_{1} a_{2}+a_{1} a_{2} a_{3}\right)$.

Согласно теореме Ньютона [11], условие отсутствия положительных корней у второго множителя в левой части уравнения (4) имеет вид: $c_{i}>0, i=1,2,3$.

Легко показать, что полиномы $c_{1}$ и $c_{3}$ положительны, если

$$
\frac{S_{0}}{N} \in\left(\frac{1+a_{1}+a_{3}+a_{1} a_{2}+a_{1} a_{3}+a_{2} a_{3}}{1+a_{3}+a_{2} a_{3}+a_{1} a_{2} a_{3}}, 1+a_{1}+a_{1} a_{2}+a_{1} a_{2} a_{3}\right),
$$

или

$$
\left\{\begin{array}{l}
\frac{S_{0}}{N} \in\left(\frac{a_{2}+a_{3}+a_{2} a_{3}+3}{a_{1}+a_{2}+a_{3}+a_{1} a_{2}+a_{2} a_{3}+a_{1} a_{2} a_{3}+3}, \frac{1+a_{1}+a_{2}-a_{1} a_{2}}{1+a_{2}+a_{1} a_{2}}\right), \\
a_{2}<1 .
\end{array}\right.
$$


Исследуем полином $c_{2}$. Для этого рассмотрим

$$
z_{2}=\frac{S_{0}}{N^{3}}\left(a_{1}+a_{2}+a_{3}+a_{1} a_{2}+a_{2} a_{3}+a_{1} a_{2} a_{3}+4\right)^{2} c_{2}
$$

как функцию от $\frac{S_{0}}{N}$. Подставим в $z_{2}$ правый и левый концы интервала (5) поочередно, получим соответственно:

$$
\begin{gathered}
z_{2}^{(1)}=\frac{a_{1}^{2} a_{2}\left(a_{1}+a_{2}+a_{3}+a_{1} a_{2}+a_{2} a_{3}+a_{1} a_{2} a_{3}+4\right)^{2}}{\left(1+a_{3}+a_{2} a_{3}+a_{1} a_{2} a_{3}\right)^{3}}\left(1+a_{3}+a_{2} a_{3}\right) \times \\
\times\left(1+a_{3}\right)\left(3+a_{1}+a_{2}+a_{1} a_{2}\right), \\
z_{2}^{(2)}=a_{1}^{2} a_{2}\left(a_{1}+a_{2}+a_{3}+a_{1} a_{2}+a_{2} a_{3}+a_{1} a_{2} a_{3}+4\right)^{2}\left(a_{1} a_{3}\left(a_{3}+1\right) a_{2}^{2}+\right. \\
\left.+\left(a_{1}+3 a_{3}+1+3 a_{1} a_{3}+a_{1} a_{3}^{2}+a_{3}^{2}\right) a_{2}+a_{1} a_{3}+a_{1}+3 a_{3}+3\right) .
\end{gathered}
$$

Таким образом, функция $z_{2}$ положительная на концах указанного интервала. Покажем, что на этом же промежутке она монотонна. Найдем производную функции $z_{2}$ по $\frac{S_{0}}{N}$ :

$$
\begin{aligned}
z_{2}^{\prime}= & 3 \frac{S_{0}^{2}}{N^{2}}\left(\left(a_{2} a_{3}+a_{2}^{2} a_{3}+a_{2}\right) a_{1}^{2}+\left(4 a_{2} a_{3}+a_{3}+2 a_{2}^{2} a_{3}+3 a_{2}+2\right) a_{1}+3 a_{2} a_{3}+\right. \\
+ & \left.a_{2}^{2} a_{3}+2 a_{2}+2 a_{3}+3\right)+2 \frac{S_{0}}{N}\left(\left(3 a_{2}-a_{3}+3 a_{2}^{2} a_{3}+3 a_{2} a_{3}+a_{2} a_{3}^{2}+a_{2}^{2} a_{3}^{2}-\right.\right. \\
- & 2) a_{1}^{2}+\left(7 a_{2} a_{3}+a_{2}^{2}+a_{3}^{2}+3 a_{2} a_{3}^{2}+5 a_{2}+2+2 a_{2}^{2} a_{3}^{2}+3 a_{2}^{2} a_{3}+2 a_{3}\right) a_{1}+ \\
& \left.+a_{2}^{2}+a_{3}^{2}+a_{2}^{2} a_{3}^{2}+a_{2} a_{3}+a_{2}+a_{3}+2 a_{2} a_{3}^{2}\right)+\left(2 a_{2}^{2} a_{3}+a_{2}^{2} a_{3}^{2}-5 a_{3}+\right. \\
& \left.+a_{2}-a_{3}^{2}-7\right) a_{1}^{2}-\left(5 a_{2}+3 a_{3}+3 a_{2}^{2} a_{3}+a_{3}^{2}+7 a_{2} a_{3}+a_{2}^{2} a_{3}^{2}+2 a_{2} a_{3}^{2}+\right. \\
& +4) a_{1}-9-11 a_{2} a_{3}-2 a_{2}^{2} a_{3}^{2}-8 a_{3}-2 a_{3}^{2}-8 a_{2}-2 a_{2}^{2}-3 a_{2}^{2} a_{3}-4 a_{2} a_{3}^{2}
\end{aligned}
$$

Повторим процедуру: подставим в $z_{2}^{\prime}$ правый и левый концы интервала (5) поочередно, получим соответственно:

$$
\begin{aligned}
& z_{2}^{\prime(1)}=\frac{a_{1}}{\left(1+a_{3}+a_{2} a_{3}+a_{1} a_{2} a_{3}\right)^{2}}\left(a_{1}+a_{2}+a_{3}+a_{1} a_{2}+a_{2} a_{3}+a_{1} a_{2} a_{3}+4\right) \times \\
& \times\left(\left(a_{2}^{3} a_{3}^{3}+3 a_{2}^{3} a_{3}^{2}+3 a_{2}^{3} a_{3}+2 a_{2}^{2} a_{3}^{3}+7 a_{2}^{2} a_{3}^{2}+9 a_{2}^{2} a_{3}+3 a_{2}^{2}+3 a_{2} a_{3}^{2}+5 a_{2} a_{3}+\right.\right. \\
& \left.+a_{2} a_{3}^{3}+3 a_{2}\right) a_{1}^{2}+\left(2 a_{2}^{3} a_{3}^{3}+7 a_{2}^{3} a_{3}^{2}+5 a_{2}^{3} a_{3}+7 a_{2}^{2} a_{3}^{3}+22 a_{2}^{2} a_{3}^{2}+25 a_{2}^{2} a_{3}+\right. \\
& \left.+6 a_{2}^{2}+6 a_{2} a_{3}^{3}+21 a_{2} a_{3}^{2}+30 a_{2} a_{3}+15 a_{2}+4 a_{3}^{2}+5 a_{3}+a_{3}^{3}+2\right) a_{1}+6+
\end{aligned}
$$




$$
\begin{gathered}
+10 a_{2}+15 a_{3}+4 a_{2}^{3} a_{3}^{2}+24 a_{2} a_{3}^{2}+3 a_{3}^{3}+27 a_{2} a_{3}+5 a_{2}^{2} a_{3}^{3}+16 a_{2}^{2} a_{3}^{2}+ \\
\left.+7 a_{2} a_{3}^{3}+2 a_{2}^{3} a_{3}+a_{2}^{3} a_{3}^{3}+16 a_{2}^{2} a_{3}+12 a_{3}^{2}+2 a_{2}^{2}\right), \\
z_{2}^{\prime(2)}=a_{1}\left(a_{1}+a_{2}+a_{3}+a_{1} a_{2}+a_{2} a_{3}+a_{1} a_{2} a_{3}+4\right)\left(\left(3 a_{2}^{3} a_{3}^{2}+3 a_{2}^{3} a_{3}+\right.\right. \\
\left.+3 a_{2}^{2} a_{3}^{2}+9 a_{2}^{2} a_{3}+3 a_{2}^{2}+3 a_{2} a_{3}+3 a_{2}\right) a_{1}^{2}+\left(3 a_{2}^{3} a_{3}^{2}+3 a_{2}^{3} a_{3}+8 a_{2}^{2} a_{3}^{2}+\right. \\
\left.+21 a_{2}^{2} a_{3}+6 a_{2}^{2}+2 a_{2} a_{3}^{2}+18 a_{2} a_{3}+15 a_{2}+a_{3}+2\right) a_{1}+6+2 a_{2} a_{3}^{2}+ \\
\left.+10 a_{2}+3 a_{3}+13 a_{2} a_{3}+2 a_{2}^{2}+2 a_{2}^{2} a_{3}^{2}+6 a_{2}^{2} a_{3}\right) .
\end{gathered}
$$

Таким образом, функция $z_{2}^{\prime}$ положительная на концах интервала (5). Покажем, что она монотонна на указанном интервале. Для этого рассмотрим производную функции $z_{2}^{\prime}$ по $\frac{S_{0}}{N}$ :

$$
\begin{gathered}
z_{2}^{\prime \prime}=6 \frac{S_{0}}{N}\left(\left(a_{2} a_{3}+a_{2}^{2} a_{3}+a_{2}\right) a_{1}^{2}+\left(4 a_{2} a_{3}+a_{3}+2 a_{2}^{2} a_{3}+3 a_{2}+2\right) a_{1}+\right. \\
\left.+3 a_{2} a_{3}+a_{2}^{2} a_{3}+2 a_{2}+2 a_{3}+3\right)+2\left(\left(3 a_{2}-a_{3}+3 a_{2}^{2} a_{3}+3 a_{2} a_{3}+a_{2} a_{3}^{2}+\right.\right. \\
\left.+a_{2}^{2} a_{3}^{2}-2\right) a_{1}^{2}+\left(7 a_{2} a_{3}+a_{2}^{2}+a_{3}^{2}+3 a_{2} a_{3}^{2}+5 a_{2}+2+2 a_{2}^{2} a_{3}^{2}+3 a_{2}^{2} a_{3}+\right. \\
\left.\left.+2 a_{3}\right) a_{1}+a_{2}+a_{3}+a_{2}^{2}+a_{3}^{2}+a_{2}^{2} a_{3}^{2}+a_{2} a_{3}+2 a_{2} a_{3}^{2}\right) .
\end{gathered}
$$

Определим точку изменения монотонности функции $z_{2}^{\prime \prime}$ :

$$
\begin{gathered}
M N=\frac{S_{0}}{N}=-\frac{1}{3}\left(\left(a_{2} a_{3}+a_{2}^{2} a_{3}+a_{2}\right) a_{1}^{2}+\left(4 a_{2} a_{3}+a_{3}+2 a_{2}^{2} a_{3}+3 a_{2}+2\right) a_{1}+\right. \\
\left.+a_{2}^{2} a_{3}+2 a_{2}+2 a_{3}+3\right)^{-1}\left(\left(3 a_{2}-a_{3}+3 a_{2}^{2} a_{3}+3 a_{2} a_{3}+a_{2} a_{3}^{2}+a_{2}^{2} a_{3}^{2}-\right.\right. \\
-2) a_{1}^{2}+\left(7 a_{2} a_{3}+a_{2}^{2}+a_{3}^{2}+3 a_{2} a_{3}^{2}+5 a_{2}+2 a_{2}^{2} a_{3}^{2}+3 a_{2}^{2} a_{3}+2 a_{3}+2\right) a_{1}+ \\
\left.+a_{2}+a_{3}+a_{2}^{2}+a_{3}^{2}+a_{2} a_{3}+2 a_{2} a_{3}^{2}+a_{2}^{2} a_{3}^{2}\right) .
\end{gathered}
$$

Сравним $M N$ с левым концом интервала (5):

$$
M N<\frac{1+a_{1}+a_{3}+a_{1} a_{2}+a_{1} a_{3}+a_{2} a_{3}}{1+a_{3}+a_{2} a_{3}+a_{1} a_{2} a_{3}} .
$$

Преобразуем полученное неравенство. Получим, что

$$
\begin{aligned}
& -\frac{1}{3}\left(\left(a_{2}+a_{2} a_{3}+a_{2}^{2} a_{3}\right) a_{1}^{2}+\left(2 a_{2}^{2} a_{3}+3 a_{2}+a_{3}+4 a_{2} a_{3}+2\right) a_{1}+3 a_{2} a_{3}+\right. \\
+ & \left.a_{2}^{2} a_{3}+2 a_{2}+2 a_{3}+3\right)^{-1}\left(1+a_{3}+a_{2} a_{3}+a_{1} a_{2} a_{3}\right)^{-1}\left(\left(9 a_{2}^{2} a_{3}+2 a_{2} a_{3}^{2}+\right.\right. \\
& \left.+3 a_{2}^{3} a_{3}+a_{2}^{3} a_{3}^{3}+6 a_{2}^{2} a_{3}^{2}+3 a_{2}+3 a_{2}^{2}+4 a_{2} a_{3}+3 a_{2}^{3} a_{3}^{2}+a_{2}^{2} a_{3}^{3}\right) a_{1}^{3}+ \\
+ & \left(26 a_{2}^{2} a_{3}^{2}+20 a_{2} a_{3}^{2}+36 a_{2} a_{3}+2 a_{2} a_{3}^{3}+3 a_{2}^{3} a_{3}^{3}+9 a_{2}^{2}+5 a_{2}^{2} a_{3}^{3}+21 a_{2}+\right. \\
& \left.+6 a_{3}+9 a_{2}^{3} a_{3}^{2}+2 a_{3}^{2}+35 a_{2}^{2} a_{3}+7 a_{2}^{3} a_{3}+4\right) a_{1}^{2}+\left(34 a_{2}^{2} a_{3}^{2}+37 a_{2} a_{3}^{2}+\right.
\end{aligned}
$$

(C) С.В. Чернышенко, Р.В. Рузич, 2015 


$$
\begin{gathered}
+5 a_{2}^{3} a_{3}+9 a_{2}^{3} a_{3}^{2}+29 a_{2}+62 a_{2} a_{3}+17+7 a_{2}^{2}+a_{3}^{3}+7 a_{2}^{2} a_{3}^{2}+12 a_{3}^{2}+ \\
\left.+3 a_{2}^{3} a_{3}^{3}+5 a_{2} a_{3}^{3}+28 a_{3}+37 a_{2}^{2} a_{3}\right) a_{1}+9+14 a_{2}^{2} a_{3}^{2}+19 a_{2} a_{3}^{2}+a_{3}^{3}+ \\
+3 a_{2}^{3} a_{3}^{2}+3 a_{2} a_{3}^{3}+11 a_{2}^{2} a_{3}+a_{2}^{3} a_{3}+26 a_{2} a_{3}+a_{2}^{3} a_{3}^{3}+16 a_{3}+3 a_{2}^{2} a_{3}^{3}+ \\
\left.+a_{2}^{2}+7 a_{2}+8 a_{3}^{2}\right)<0 .
\end{gathered}
$$

Таким образом, функция $z_{2}^{\prime \prime}$ монотонна на интервале (5), и, следовательно, функции $z_{2}^{\prime}$ и $z_{2}$ тоже монотонны на этом интервале. Поскольку функция $z_{2}$ монотонная на интервале (5) и положительна на его концах, то полином $c_{2}$ положительный на этом интервале. Используя этот же метод, легко показать, что полином $c_{2}$ отрицательный на интервале (6). Следовательно, точка $M$ устойчива лишь в промежутке (5).

Итак, на примере четырехмерной модели разомкнутого гиперцикла Эйгена удалось показать, что теоретически рассмотренная экосистема в процессе эволюции проходит $n+1$ этап.

\section{Выводы}

В работе рассмотрена многомерная модель разомкнутого гиперцикла Эйгена. Показано, что исследование $n$-мерной системы может быть сведено к исследованию систем меньшей размерности. Доказано, что первые $(n-2)$ бифуркационные условия для $n$-мерной модели разомкнутого гиперцикла Эйгена совпадают с первыми $(n-2)$ бифуркационными условиями для модели размерности $(n-1)$.

Экологическая сукцессия, для описания которой используется $n$-мерная модель разомкнутого гиперцикла Эйгена, имеет потенциально $(n+1)$ стадию. Первая из них соответствует очень небольшому размеру экологической ниши $\left\{0<S_{0}<\left(1+a_{1}\right)^{-1} N\right\}$, и в экологической макросистеме может существовать только одна ассоциация. Каждая новая стадия (кроме последней) означает появление новой ассоциации в экосистеме. Перестройка структуры системы является, с математической точки зрения, последовательностью бифуркаций в положительной области фазового пространства модели и определяет квазидискретную динамику последней.

1. Clements F.E. Plant Succession: Analysis of the Development of Vegetation / F.E. Clements. — Washington D.C.: Publ. Carnegi Inst., 1916. - 512p.

2. Aaviksoo K. Simulating Vegetation Dynamics and Land Use in a Mire Landscape Using a Markov Model / K. Aaviksoo // Landscape and Urban Planning. - 1995. - Vol. 31. P. $129-142$.

3. Логофет Д.О. Неоднородные марковские модели сукцессии растительности: новые перспективы старой парадигмы / Д.О. Логофет, Е.А. Денисенко, Л.Л. Голубятников // Известия РАН. Серия биологическая. - 1997. — № 5. - С. 613-622.

4. Исаев А.С. Сукцессионные процессы в лесных сообществах: модели фазовых переходов / А.С. Исаев, В.Г. Суховольский, А.И. Бузыкин, Т.М. Овчинникова // Хвойные бореальной зоны. — 2008. - Том XXV, № 1-2. - С. 9-16. 
5. Connell J.H. Mechanisms of Succession in Natural Communities and Their Role in Community Stability and Organization / J.H. Connell, R.O. Slatyer // The American Naturalist. - 1977. - Vol. 111. - P. 1119-1144.

6. Chakrabarti C.G. Non-equilibrium Thermodynamics of Lotka-Volterra Ecosystems: Stability and Evolution / C.G. Chakrabarti, S. Ghosh, S. Bhadra // Journal of Biological Physics. 1995. - Vol. 21. - P. 273-284.

7. McNauhghton S.J. Dominance and the Niche in Ecological Systems / S.J. McNauhghton, L.L. Wolf // Science. — 1970. - Vol. 167. — P. 131-139.

8. Работнов А.Т. Фитоценология / А.Т. Работнов - [3-е изд., перераб. и доп.]. — М. : Издво МГУ, 1992. — $352 \mathrm{c}$.

9. Чернышенко С.В. Нелинейные методы динамики лесных биогеоценозов / С.В. Чернышенко. - Днепропетровск: Изд-во ДНУ, 2005. — 500 с.

10. Chernyshenko S.V. Bifurcation Model of Successions in Ecosystems // S.V. Chernyshenko, R.V. Ruzich // ECMS2013, 27-th European conference on modelling and simulation, Alesund, Norway, May 27-30, 2013. - 2013. - P. 767-774.

11. Березич И.С. Методы вычислений / И.С. Березич, Н.П. Жидков: В 2 т. - М. : Гл. изд. физ.-мат. лит-ры, 1959. - Т. 2. - 620 с.

UDC 517.925.5

\section{DISCRETE EFFECTS IN CONTINUOUS MODELS OF SUCESSIONS}

\section{S.V. Chernyshenko, R.V. Ruzich \\ Khmelnitsky National University}

Introduction. In article a long-term ecological successions are considered. It is step-by-step process. The continuous model (model of open Eigen's hypercycle) is used to describe this process.

The purpose of the paper is to investigate non-linear properties of the system, which define discrete processes that occur in the one.

Results. The multi-dimension case of the model of open Eigen's hypercycle has been analyzed. It is shown that in many cases the consideration of dynamics of the $n$-dimensional system can be simplified by partial reduction to $(n-1)$-dimensional cases.

It is mathematically shown that evolution of system, which is described by the $n$-dimensional model of open Eigen's hypercycle has, as maximum, $n+1$ stages. Presence and duration of each stage are determined by the size of the ecological niche, as a characteristics of the environment. As an example: if the niche is very small $\left(0<S_{0}<\left(1+a_{1}\right)^{-1} N\right)$, there is only one association in the stable state of the ecosystem.

Conclusion. It is shown that the continuous model can describe discrete processes of sucessions. The quasi-discrete dynamics of the system is explained by its bifurcation properties, produced step-by-step changing of the system structure.

Keywords: succession, discrete process, continuous model, Eigen's hypercycle, bifurcation.

1. Clements F.E. Plant Succession: Analysis of the Development of Vegetation. Washington D.C.: Publ. Carnegi Inst. 1916. 512p. 
2. Aaviksoo K. Simulating Vegetation Dynamics and Land Use in a Mire Landscape Using a Markov Model. Landscape and Urban Planning, 1995, vol. 31, pp. 129-142.

3. Logofet D.O., Golubyatnikov L.L., Denisenko E.A. Nonhomogeneous Markov Model of Vegetation Succession: A New Perspective of the Old Paradigm. Izvestiya RAS. Biology Series, 1997, no. 5, pp. 613-622 (in Russian).

4. Isaev A.S., Suhovolsky V.G., Buzykin A.I., Ovchinikov T.M. Successional Processes in Forest Communities: Models of Phase Transitions. Khvojnyje borealnoy zony, 2008, vol. XXV, no. 1-2, pp. 9-16 (in Russian).

5. Connell J.H., Slatyer R.O. Mechanisms of Succession in Natural Communities and Their Role in Community Stability and Organization. The American Naturalist, 1977, vol. 111, pp. 1119-1144.

6. Chakrabarti C.G., Ghosh S., Bhadra S. Non-equilibrium Thermodynamics of Lotka-Volterra Ecosystems: Stability and Evolution. Journal of Biological Physics, 1995, vol. 21, pp. 273-284.

7. McNauhghton S.J., Wolf L.L. Dominance and the Niche in Ecological Systems. Science, 1970, vol. 167, pp. 131-139.

8. Rabotnov A.T. Phytocenology. Moscow: MGU Press. 1992. 352 p. (in Russian).

9. Chernyshenko S.V. Nonlinear Analysis of Forest Ecosystems Dynamics. Dnepropetrovsk: Dnepropetrovsk University Press. 2005. 500 p. (in Russian).

10. Chernyshenko S. V., Ruzich R. V. Bifurcation Model of Successions in Ecosystems. Proceedings of 27th European Conference on Modelling and Simulation ECMS 2013. May 27-30 2013. Alesund, Norway, pp. 767-774.

11. Berezich I. S., Zitkov N. P. Methods of Calculations. Vol. 1-2. Moscow: Phys.-math. Liter. Main Press, 1959. 620 p. (in Russian).

Получено 17.03.2015 


\section{Доценко Сергей Иванович,}

кандидат физико-математических наук, старший научный сотрудник, доцент кафедры исследования операций факультета кибернетики Киевского национального университета имени Тараса Шевченко, пр. Глушкова, 4 Д, г. Киев, 03680, e-mail: sergei204@ukr.net

Волков Александр Евгеньевич, младший научный сотрудник отдела интеллектуального управления Международного научно-учебного центра информационных технологий и систем НАН и МОН Украины, пр. Глушкова, 40, Киев, 03680.

e-mail: alexvolk@ukr.net

\section{Волоченюк Дмитрий Александрович,} младший научный сотрудник отдела интеллектуального управления Международного научно-учебного центра информационных технологий и систем НАН и МОН Украины, пр. Глушкова, 40, Киев, 03680.

e-mail: P-h-o-e-n-i-x@ukr.net

\section{Коиур Максим Петрович,}

ассистент кафедры математических проблем управления и кибернетики Черновицкого национального университета имени Юрия Федьковича, г. Черновцы, ул. Коцюбинского, 2, 58012.

e-mail: piramidam@rambler.ru

\section{Мащенко Сергей Олегович,}

доктор физико-математических наук, профессор, профессор кафедры системного анализа и теории принятия решений Киевского национального университета имени Тараса Шевченко, пр. Глушкова, 4 Д, г. Киев, 03680, e-mail: msomail@yandex.ua
Наконечный Александр Григорьевич, доктор физико-математических наук, профессор, академиксекретарь отделения кибернетики и системного анализа, заведующий кафедрой системного анализа и теории принятия решений Киевского национального университета имени Тараса Шевченко, пр. Глушкова, 4 Д, г. Киев, 03680, e-mail: a.nakonechniy@gmail.com

Павлов Вадим Владимирович,

доктор технических наук, профессор, заведующий отделом интеллектуального управления Международного научно-учебного центра информационных технологий и систем НАН и МОН Украины, ул. Академика Глушкова, 40, Киев, 03680. e-mail: dep185@irtc.org.ua

Рузич Роман Васильевич, ассистент кафедры прикладной математики и социальной информатики Хмельницкого национального университета, г. Хмельницкий, ул. Институтская, 11, 29016 email: ninasus@gmail.com

Савченко Евгения Анатольевна,

кандидат технических наук, старший научный сотрудник отдела информационных технологий индуктивного моделирования Международного научно-учебного центра информационных технологий и систем НАН Украины и МОН Украины, пр. Глушкова 40, г. Киев, 03680 ГСП, email: savchenko_e@meta.ua 
Суровцев Игорь Викторович,

кандидат технических наук, старший научный сотрудник отдела системного моделирования Международного научно-учебного центра информационных технологий и систем НАН Украины и МОН Украины, пр. Глушкова 40, г. Киев, 03680 ГСП, email: igorsur52@gmail.com

Чернылиенко Сергей Викторович, доктор биологических наук, кандидат физико-математических наук, профессор, заведующий кафедрой прикладной математики и социальной информатики Хмельницкого национального университета, г. Хмельницкий, ул. Институтская, 11, 29016, email: svc@a-teleport.com
Шушарин Юрий Викторович,

кандидат физико-мататематических наук, доцент кафедры высшей математики Киевского национального экономического университета им. Вадима Гетьмана, пр. Победы, 54/1, г. Киев, 03680,

e-mail: shusharin@meta.ua 


\section{ВНИМАНИЮ АВТОРОВ!}

\section{Требования к рукописям статей}

1. Рукопись представляется на белой бумаге в двух экземплярах (язык русский, 9-10 с.) и электронная версия. К рукописи прилагаются:

- аннотации - на русском и украинском языках (УДК, фамилия, инициалы автора/ов, название статьи, 5-6 строк текста, ключевые слова), на английском языке (фамилия, инициалы автора/ов, место работы, город, страна, название статьи, от 250 слов, с выделением рубрик: введение, цель, результаты, выводы, ключевые слова);

— согласие автора/ов на публикацию;

- сведения об авторе должны включать: ФИО, ученую степень, научное звание, должность, отдел, место работы, почтовый адрес организации, телефон (служ., моб., дом.), E-mail.

2. Текст статьи подается с обязательными рубриками: введение, постановка задачи, цель, результаты, четко сформулированные выводы.

3. Поданные статьи проходят рецензирование ведущими специалистами в данной области.

\section{Структура статьи в КВТ}

Удк
НАЗВАНИЕ
И.О.Фамилия / и
Организация/и

Аннотация (русс., 5-10 строк)

Ключевые слова: (5-8 слов)

Анотація (укр.., 5-10 стр)

Ключові слова: (5-8 слів)

\section{Введение}

Цель

Постановка задачи

Результаты (1-3 раздела с названиями)

\section{Выводы}

Список литературы на языке оригинала (в порядке упоминания в тексте, по стандарту ДСТУ ГОСТ 7.1 : 2006, ДСТУ ГОСТ 7.80 : 2007).

Abstract 0,5-0,7 стр.

ФИО, Название статьи на анг языке

Название организации - на анг языке.

С обязательными рубриками:

Introduction.

Purpose.

Results.

\section{Conclusion.}

Keywords:

Список литератиры - перевод источников на английский язык, фамилии и инициалы авторов - транслитерация

(Author A.A. Article. Journal, 2000, vol. 1, no. 2, pp. 111-112.

Author A.A., Author B.B. Book. City: Publisher, 2000. 111 p. (in Russian). 


\section{Требования к текстовому файлу}

Формат файла *.doc, *.rtf. Файл должен быть подготовлен с помощью текстового редактора Microsoft Word 2003.

Используемые стили: шрифт Times New Roman, высота 12 пт, межстрочное расстояние - полуторное. Формат бумаги A4, поля (слева, справа, вверху, внизу) $2 \mathrm{~cm}$.

Формульл набираются в редакторе формул Microsoft Equation Editor 3.0.

Опции редактора формул - $(10,5 ; 8,5 ; 7,5 ; 14 ; 10)$. Ширина формул не более $12 \mathrm{~cm}$.

Рисунки должны быть достаточно качественными, созданы встроенным редактором рисунков Word Picture либо иными Windows-приложениями (в этом случае рисунки должны быть представлены отдельными файлами соответствующих форматов). Ширина рисунков не более $12 \mathrm{~cm}$. «Таблица».

Таблицы выполняются стандартным встроенным в Word инструментарием

В розничную продажу журнал не поступает

\section{Оформление подписки для желающих опубликовать статьи в нашем журнале обязательно.}

Подписка осуществляется:

- „Каталог видань України”, подписной индекс 86598

- в агентстве «Укринформнаука» НАН Украины ukrinformnauka@gmail.com

Підп. до друку 30.06.2015. Формат 70×108/16. Папір офсетний. Ум. друк. арк. 10,33. Обл. вид. арк. 9,84. Тираж 100. Зам. №3211

Видавничий дім “Академперіодика” НАН України 01004, Київ 4, вул. Терещенківська, 4.

Свідоцтво про внесення до Державного реєстру суб’єкта видавничої справи

Серії ДК №544 від 27.07.2001 p. 